\title{
UAV Assistance Paradigm: State-of-the-art in Applications and Challenges
}

\author{
Bander Alzahrani, Member, IEEE, Omar Sami Oubbati, Member, IEEE, \\ Ahmed Barnawi, Member, IEEE, Mohammed Atiquzzaman, Senior Member, IEEE, \\ and Daniyal Alghazzawi, Senior Member, IEEE
}

\begin{abstract}
Unmanned Aerial Vehicles (UAVs) are an emerging technology with the potential to be used in industries and various sectors of human life to provide a wide range of applications and services. During the last decade, there has been a growing focus of research in the UAV's assistance paradigm as a fundamental concept resulting in the constant improvement between different kinds of ground networks and the hovering UAVs in the sky. Recently, the wide availability of embedded wireless interfaces in the communicating entities has allowed the deployment of such a paradigm simpler and easiest. Moreover, due to UAVs' controlled mobility and adjustable altitudes, they can be considered as the most appropriate candidate to enhance the performance and overcome the restrictions of ground networks. This comprehensive survey both studies and summarizes the existing UAV-assisted research, such as routing, data gathering, cellular communications, Internet of Things (IoT) networks, and disaster management that supports existing enabling technologies. Descriptions, classifications, and comparative studies related to different UAV-assisted proposals are presented throughout the paper. By pointing out numerous future challenges, it is expected to simulate research in this emerging and hot research area. To the best of our knowledge, there are many survey papers on the topic from a technology perspective. Nevertheless, this survey can be considered as the first attempt at a comprehensive analysis of different types of existing UAV-assisted networks and describes the state-of-the-art in UAV-assisted research.
\end{abstract}

Index Terms-UAV; IoT; Cellular communications; Routing; Data gathering; Fog computing.

\section{INTRODUCTION}

The last decade has witnessed an explosive growth in the use of Unmanned Aerial Vehicles (UAVs) in every facet of life. According to the report from the Federal Aviation Administration (FAA), the number of UAVs will reach 3.2 million flying units by 2022 [1]. This will lead to the emergence of new use cases of such vehicles and also provide new business opportunities for telecommunication operators [2]. A wide range of tasks can be performed by UAVs creating many useful applications, such as Internet access or Phone call, while enhancing the measurement of ground end-users' satisfaction or what is called quality of experience (QoE) [3]. The UAV assistance paradigm, which uses UAVs

BA. Alzahrani, A. Barnawi, and D. Alghazzawi are with Faculty of computing and IT, King Abdulaziz University, Jeddah, Saudi Arabia. E-mails: (baalzahrani@kau.edu.sa; ambarnawi@kau.edu.sa; dghazzawi@kau.edu.sa)

O. S. Oubbati is with the Computer Science and Mathematics Laboratory, University of Laghouat, BP 37G, Ghardaïa Road, Laghouat 03000, Algeria. E-mail: s.oubbati@lagh-univ.dz

M. Atiquzzaman is with the University of Oklahoma, Norman, OK USA. E-mail: atiq@ou.edu to support many applications and terrestrial networks, users, and communicating entities is, thus experiencing a rapid increase in research interests. The benefits of using the UAV assistance concept are to provide a cost-effective solution for terrestrial networks' challenges, an efficient eye in the sky of the covered area, reliable coordination with ground communication devices, a robust backup network for damaged ground network infrastructures, and a communication bridge between isolated users and existing cellular networks. The relationship between UAVs and ground entities is crucial to accomplish different assigned activities, from surveillance to the enhancement of ground cellular networks' coverage. Also, other kinds of applications involving UAVs have been designed in order to enhance the connectivity of ground networks [4], coordinate with ground vehicles [5], collect data from ground sensors [6], recover connectivity in harsh environments [7], and secure communications with the ground [8]. All these applications are required to guarantee minimum reliability towards the ground in order to both provide near optimal service and fully exploit the potential of UAVs. However, there still exists numerous technical issues and challenges during the deployment of UAVs, which remain not fully investigated and solved. Furthermore, different requirements related to UAVs are neglected, such as their high mobility, their restrictedenergy capacity, and their optimal placement. Consequently, a strong knowledge of the existing UAV-assisted solutions is required to address the existing issues and to enhance the functionality of applications.

\section{A. Motivation}

The assistance of UAVs to different ground entities or terrestrial networks is recently considered as the keystone to the success of an important number of tasks requiring a deep improvement in terms of completion time, network performance, and flexibility. Therefore, a well-organized concept of this paradigm needs to be accurately defined while considering the different requirements related to UAVs. This allows UAVs to better serve ground users (GUs) and to successfully accomplish the assigned tasks [9]. However, each kind of task has its own challenges and difficulties [10]. For instance, UAVs have the ability to bridge the communication gaps of ground networks [11], to substitute the damaged or overloaded terrestrial base stations (BSs) [12], and to monitor hostile environments or disaster areas [13]. In addition to the existing UAV challenges, a number of new challenges, such 
as technical and standardization aspects [14], public safety and privacy [15], and mobility optimization [16] need further attention. As a consequence, an important number of UAVassisted techniques have been proposed across the literature trying, at each time, to bring new functionalities and concurrent performances, while overcoming the aforementioned constraints. The potential benefits of UAVs give rise to the following queries:

- When is it beneficial to deploy a communication network of UAVs over a terrestrial network?

- What is the optimal number of UAVs and the mobility models to be adopted for a given scenario?

- How can UAVs enhance the performances of ground networks and better serve GUs?

Motivated by the aforementioned questions, in this survey, we provide a detailed explanation of the existing achievements and proposals of UAV-assisted networks. These solutions can be categorized according to the nature of UAV missions, such as routing, UAV and Unmanned Ground Vehicle (UGV)/Internet of Things (IoT) devices coordination, cellular communication, disaster management, computing, data gathering, monitoring, and secure communication. To the best of our knowledge, our survey is the first to provide a comprehensive classification of UAV-assisted networks.

\section{B. Limitations of the literature and contributions}

A number of good surveys have been published on UAVassisted networks, such as UAV-assisted cellular networks, UAV-UGV coordination, UAV-IoT networks, and UAV-assisted disaster management. Table I provides a brief comparative study based on four parameters between the most popular surveys in the literature and our survey. Indeed, extensive analysis is performed for each survey based on several points, such as the number of discussed UAV-assisted applications, the popularity in terms of citations, the number of comparative studies, the discussion of future challenges, and the main description. Since none of these surveys had covered all kinds of UAV assistance use cases, it is time to present a comprehensive up-to-date review of this important paradigm in a single paper. Specifically, our contributions can be delineated as:

- We provide both an overview of the UAV assistance paradigm and in-depth discussions on its main challenges and constraints.

TABLE I: Analysis of UAV assistance surveys proposed in the literature.

\begin{tabular}{|c|c|c|c|c|c|c|}
\hline & Surveys & Application use cases & Popularity & Comparisons & Future challenges & Description \\
\hline \multirow{3}{*}{2019} & Ref. [17] & - UAV Cellular Communications. & New & $\sqrt{ }$ & 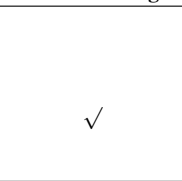 & $\begin{array}{l}\text { Surveyed all the developments that help to integrate UAVs } \\
\text { into cellular networks by highlighting different aspects, such } \\
\text { as the available kinds of UAVs, the interference issues, } \\
\text { UAVs as BSs and relays, the adopted test-beds, and the } \\
\text { cyber-physical security communication schemes. In addition, } \\
\text { a set of open research problems are highlighted in the end } \\
\text { of this survey. }\end{array}$ \\
\hline & Ref. [18] & $\begin{array}{l}\text { - Air-to-Ground Channel modeling. } \\
\text { - Optimal Deployment of UAVs. } \\
\text { - Trajectory Optimization. } \\
\text { - Resource Management and Energy Efficiency. }\end{array}$ & High & $\sqrt{ }$ & $\sqrt{ }$ & $\begin{array}{l}\text { Presented a comprehensive survey on the benefits of using } \\
\text { UAVs in wireless communications by investigation } \\
\text { important UAV challenges, such as the optimal deployment, } \\
\text { channel modeling, energy efficiency, and different analytical } \\
\text { and mathematical frameworks. Furthermore, this survey is } \\
\text { concluded by outlining a set of future research directions } \\
\text { related to the topic. }\end{array}$ \\
\hline & Ref. [19] & - Drone-Assisted Vehicular Networks & New & $x$ & $\sqrt{ }$ & $\begin{array}{l}\text { Studied and analyzed three aspects of UAV safety: (i) } \\
\text { Sensors, (ii) Exchange of information, and (iii) Multi-UAVs } \\
\text { applications. This survey also discussed future research } \\
\text { directions. }\end{array}$ \\
\hline \multirow{7}{*}{2018} & Ref. [20] & - Security and Privacy Issues of UAV. & Medium & $x$ & $\sqrt{ }$ & $\begin{array}{l}\text { Proposed a detailed architecture of UAV-assisted VANET } \\
\text { and highlighted the improvements provided by UAVs. } \\
\text { Moreover, this survey presented important key challenges, } \\
\text { research directions, and studied a given scenario to } \\
\text { demonstrate the effectiveness of this kind of collaboration. }\end{array}$ \\
\hline & Ref. [21] & - Channel Modeling for UAV Communications. & Medium & $\sqrt{ }$ & $\sqrt{ }$ & $\begin{array}{l}\text { Provided a comprehensive study of the measurement } \\
\text { techniques dedicated to UAV channel modeling and } \\
\text { discussed numerous channel characterization solutions. } \\
\text { Furthermore, this survey outlined future research directions } \\
\text { of this topic. }\end{array}$ \\
\hline & Ref. [22] & - UAV Swarm Architectures. & Low & $x$ & $x$ & $\begin{array}{l}\text { Investigated UAV swarm in the context of cellular mobile } \\
\text { network infrastructure. Also, it described an initial test-bed } \\
\text { development while considering different limitation factors } \\
\text { related to UAVs. }\end{array}$ \\
\hline & Ref. [23] & - UAV Monitoring. & Low & $\sqrt{ }$ & $x$ & $\begin{array}{l}\text { Investigated four types of UAVs and compare them } \\
\text { according to several criteria, and especially their ability to } \\
\text { carry out tasks under adverse weather. }\end{array}$ \\
\hline & Ref. [24] & - UAV Swarm Architectures. & Low & $x$ & $x$ & $\begin{array}{l}\text { Surveyed UAV swarm formation techniques and their } \\
\text { integration into cellular networks. Moreover, it proposed a } \\
\text { new swarm architecture and described an initial test bed } \\
\text { development. }\end{array}$ \\
\hline & Ref. [25] & $\begin{array}{l}\text { - UAV-UGV coordination. } \\
\text { - UAV-Assisted WSN. } \\
\text { - UAV-Assisted Ground Nodes. } \\
\text { - Coverage Optimization. }\end{array}$ & Low & $\sqrt{ }$ & $\sqrt{ }$ & $\begin{array}{l}\text { Briefly investigated the most important challenges related to } \\
\text { different kinds of applications in designing Internet of } \\
\text { Flying Robot (IoFR). In addition, it is also focused on } \\
\text { several designing challenges, such as connectivity, path } \\
\text { planning, energy efficiency, and collision avoidance. }\end{array}$ \\
\hline & Ref. [26] & $\begin{array}{l}\text { - UAV Networking Layer Techniques. } \\
\text { - UAV Physical Layer Techniques. } \\
\text { - UAV-aided B5G Networks. } \\
\text { - Joint Communication, Computing, and } \\
\text { Caching. }\end{array}$ & High & $\sqrt{ }$ & $\sqrt{ }$ & $\begin{array}{l}\text { Briefly introduced space-air-ground integrated networks and } \\
\text { related challenges. Also, it provided a comprehensive review } \\
\text { of various UAV-based } 5 \mathrm{G} \text { techniques categorized by } \\
\text { different domains. }\end{array}$ \\
\hline
\end{tabular}


TABLE I: (Cont.) Analysis of UAV assistance surveys proposed in the literature.

\begin{tabular}{|c|c|c|c|c|c|c|}
\hline \multicolumn{2}{|r|}{ Surveys } & Application use cases & Popularity & Comparisons & Future challenges & Description \\
\hline \multirow{5}{*}{2017} & Ref. [27] & $\begin{array}{l}\text { - UAV Data Collection. } \\
\text { - UAV Network Layer Techniques. }\end{array}$ & Medium & $\sqrt{ }$ & - & $\begin{array}{l}\text { Studied the services and requirements of UAV-based } \\
\text { systems, presented the different adopted networking } \\
\text { architectures, and highlighted a number of protocols used in } \\
\text { networking layers. Moreover, it discussed both the } \\
\text { middleware layer services and the involvement of UAVs as } \\
\text { data collectors in Wireless Sensor Networks (WSNs). }\end{array}$ \\
\hline & Ref. [28] & $\begin{array}{l}\text { - Distributed Gateway Selection. } \\
\text { - UAV Network Layer Techniques. }\end{array}$ & High & $\sqrt{ }$ & $\sqrt{ }$ & $\begin{array}{l}\text { Studied relevant gateway selection mechanisms and } \\
\text { cloud-based stability-control techniques. }\end{array}$ \\
\hline & Ref. [29] & - Cyber Security in UAV Networks. & Low & $\sqrt{ }$ & $\sqrt{ }$ & $\begin{array}{l}\text { Studied the existing cyber-security vulnerabilities for UAVs } \\
\text { by focusing on actual and simulated attacks and the } \\
\text { involvement of UAVs. }\end{array}$ \\
\hline & Ref. [30] & - UAV Regulations. & Medium & $\sqrt{ }$ & $\sqrt{ }$ & $\begin{array}{l}\text { Highlighted perspectives on UAV regulations and presented } \\
\text { the status of UAV regulations in different countries and } \\
\text { across time. }\end{array}$ \\
\hline & Ref. [31] & $\begin{array}{l}\text { - UAV Remote Sensing } \\
\text { - UAV Regulations }\end{array}$ & Low & $\sqrt{ }$ & $x$ & $\begin{array}{l}\text { Investigated UAV systems technology and their features, } \\
\text { such as data processing, payloads, and propellers. Moreover, } \\
\text { it evaluated some applications related to different services } \\
\text { and it reviewed the existing UAV regulations. }\end{array}$ \\
\hline \multirow{3}{*}{2016} & Ref. [32] & - UAV disaster Management & Medium & $\sqrt{ }$ & $\sqrt{ }$ & $\begin{array}{l}\text { Identified the relevant disaster management applications } \\
\text { involving UAVs and discussed open research challenges } \\
\text { related to this kind of applications. }\end{array}$ \\
\hline & Ref. [33] & $\begin{array}{l}\text { - UAV Routing. } \\
\text { - UAV Handover. } \\
\text { - Energy Efficiency. }\end{array}$ & High & $\sqrt{ }$ & $\sqrt{ }$ & $\begin{array}{l}\text { Studied different issues related to UAV communication } \\
\text { networks, such as routing, multi-UAV architecture } \\
\text { characterization, and seamless handover. }\end{array}$ \\
\hline & Ref. [34] & $\begin{array}{l}\text { - Search and Rescue. } \\
\text { - UAV Coverage. } \\
\text { - UAV Construction. } \\
\text { - Delivery of goods. }\end{array}$ & High & $\sqrt{ }$ & $\sqrt{ }$ & $\begin{array}{l}\text { Studied the different requirements of UAV networks for } \\
\text { civil application from networking and communications } \\
\text { viewpoint. Also, it reported experimental results from } \\
\text { existing projects and it investigated existing communication } \\
\text { technologies to support UAV networks. }\end{array}$ \\
\hline \multirow[b]{2}{*}{2015} & Ref. [35] & - UAV Traffic Monitoring & High & $\sqrt{ }$ & $x$ & $\begin{array}{l}\text { Surveyed the most relevant UAV-based systems for traffic } \\
\text { monitoring. }\end{array}$ \\
\hline & Ref. [36] & $\begin{array}{l}\text { - UAV Radio Technology. } \\
\text { - UAV Monitoring. }\end{array}$ & High & $\sqrt{ }$ & $\sqrt{ }$ & $\begin{array}{l}\text { Studied different issues, challenges, and future perspectives } \\
\text { related to the association between UAVs and cognitive radio } \\
\text { technology. }\end{array}$ \\
\hline \multicolumn{2}{|c|}{ Our survey } & $\begin{array}{l}\text { - UAV-UGV Coordination. } \\
\text { - UAV Routing. } \\
\text { - UAV Data Gathering. } \\
\text { - UAV Monitoring. } \\
\text { - UAV Cellular Communications. } \\
\text { - UAV-IoT Networks. } \\
\text { - UAV Disaster Management. } \\
\text { - UAV Computing. } \\
\text { - UAV Secure Communications. }\end{array}$ & -- & $\sqrt{ }$ & $\sqrt{ }$ & $\begin{array}{l}\text { Presents a comprehensive survey of the most important } \\
\text { UAV assistance schemes supporting different enabling } \\
\text { technologies proposed across the literature. Moreover, } \\
\text { several comparative studies, descriptions, and classifications, } \\
\text { are presented. }\end{array}$ \\
\hline
\end{tabular}

- We cover the majority of existing UAV-assisted network schemes based on nine (9) categories.

- We study the functionality of each scheme while highlighting its major shortcomings.

- We present a detailed comparative study in each category based on crucial parameters to help readers getting a quickly high-level understanding of the proposed solutions.

- We summarize the survey by both discussing the different research challenges and proposing some solutions and recommended references to address them. Also, we highlight some additional insights at the conclusion of this survey.

\section{Organization of the survey}

A graphical depiction of the organization of our survey is shown in Fig. 1. This figure can be read as a diagram comprising three hierarchical rings summarizing the skeleton of this survey. The first ring indicates the core of this work, which focuses on the UAV assistance paradigm. In the second ring, nine (9) main selected types of UAV-assisted applications (i.e., the sections' titles are mentioned) are discussed and the open research challenges are analyzed and studied. In the third ring, the subcategories under each kind of UAV-assisted applications are discussed. Each subcategory corresponds to each subsection belonging to a given category (i.e., each section has many subsections).

In the following, a brief description of the content of each section:

- In Section II, we define the UAV-assistance paradigm while highlighting its main challenges that arise in this kind of assistance.

- In Section III presents the major coordination techniques between UAVs and Unmanned Ground Vehicles (UGVs) with respect to four kinds of applications. In Section IV, we describe the existing routing solutions that are either designed for Vehicular Ad hoc Networks (VANETs) or ground networks. Section V describes UAV data gathering schemes in different kinds of networks.

- Section VI draws a sketch of UAV monitoring in different environments. Section VII reports the UAV-assisted cellular networks with regard to different mechanisms. 


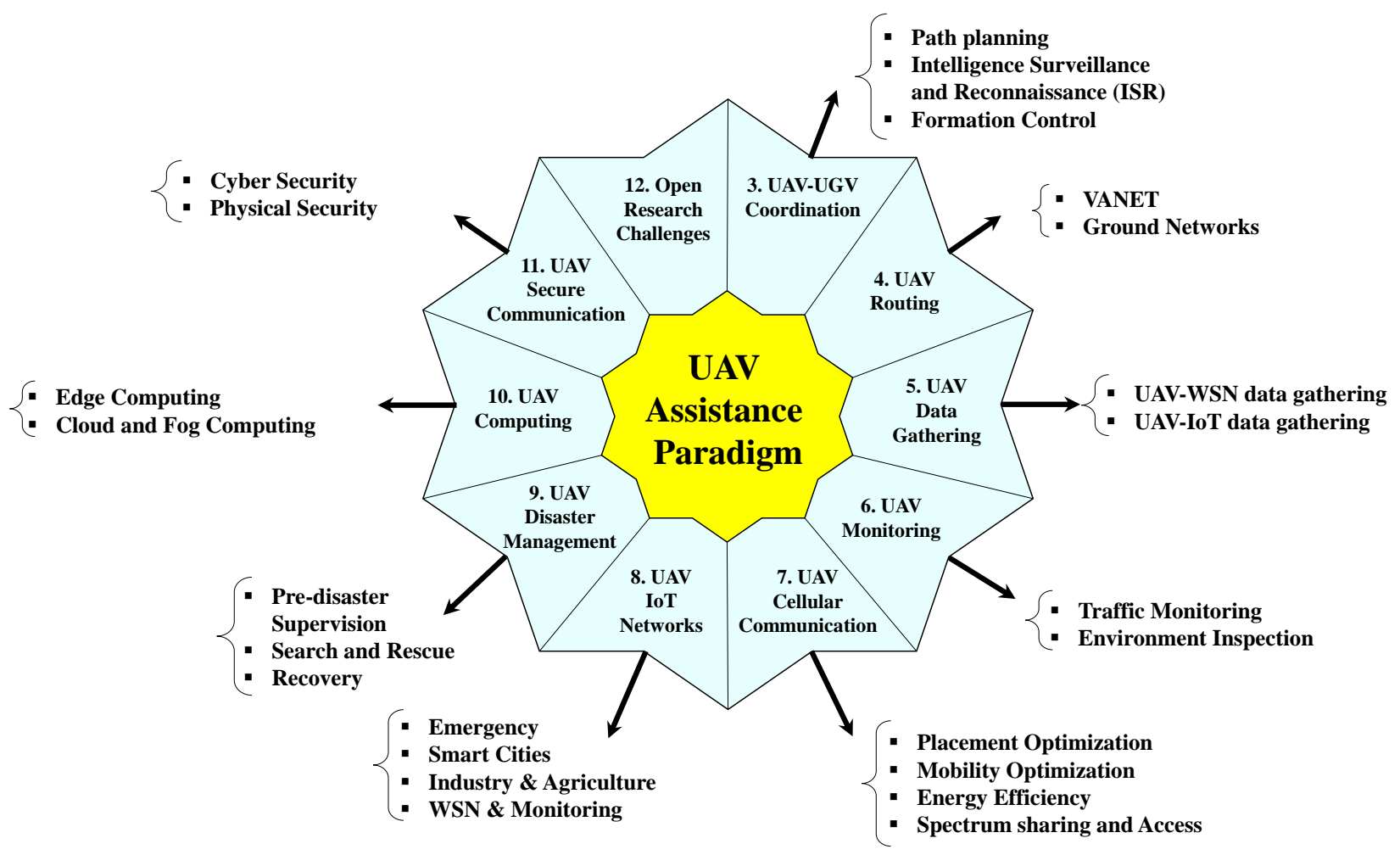

Fig. 1: Organization of the survey.

Sections IX, X, and XI, describe the assistance of UAVs to disaster management, computing, and secure communications, respectively.

- Section XII discusses lessons learned and outlines different research challenges and future perspectives faced by the UAV assistance paradigm. Moreover, this comprehensive survey is concluded at the same section with additional insights on this timely area of research.

\section{Overview of UAV Assistance Paradigm AND CHALLENGES}

The deployment of UAVs over a given terrestrial network always constitutes a challenging task. As illustrated in Fig. 2, UAVs can be quickly deployed whenever required, which makes them appropriate candidates for providing assistance to terrestrial networks, e.g., by increasing connectivity in vehicular networks, reducing handovers for device-to-device (D2D) networks, offloading traffic in cellular networks, and establishing on-demand wireless communications with GUs in disaster situations during which terrestrial networks are not fully operational. Recently, such deployment has been widely studied in the literature. For instance, in [37], the deployment of multiple UAVs as flying BSs for providing on-demand wireless service to a set of cellular users was studied. In [38], the authors investigate how to increase the worst-case secrecy of a proposed secure communication system using UAVs communicating with terrestrial networks in the presence of eavesdroppers on the ground. Furthermore, the authors in [39] presented a UAV enabled data gathering system with the aim to study the energy trade-off problem in the UAV-toground communications. Nevertheless, several challenges and issues are frequently distinguished in these works that include channel modeling, mobility planning, network security, energy supply, interference issues, etc., as discussed in the rest of this section. This section limits its focus on discussing the most important challenges, while the different UAV-assisted proposals are left for a thorough investigation in Section IV.

\section{A. Channel modeling}

Compared to traditional terrestrial communication channels, the UAV assistance paradigm introduces three different communication channels: (i) Air-to-Ground (A2G) channel, (ii) Air-to-Air (A2A) channel, and (iii) Satellite-to-Air (S2A) channel. In fact, the $\mathrm{A} 2 \mathrm{G}$ channel has its own characteristics, such as its exposition to the blockage, the impacts of UAVs' altitude, and type of the propagation environment [40]. Furthermore, the effects of shadowing caused by the UAVs' body should be considered in channel modeling. There exist several works in the literature trying to optimize transmission performance, measure channel parameters, and simulate channel. In contrast to the A2G channel, the A2A channel is based exclusively on UAVs with different communication environments. Indeed, even if A2A channel has a smaller multi-path fading compared to the $\mathrm{A} 2 \mathrm{G}$ channel, it can be impacted by Line-of-Sight (LoS) components and ground reflection like A2G channel [41]. Moreover, a larger Doppler effect is distinguished in the A2A channel, which is due to the potentially significant relative speed between the UAVs [42]. To address these issues, there is a crucial need to 


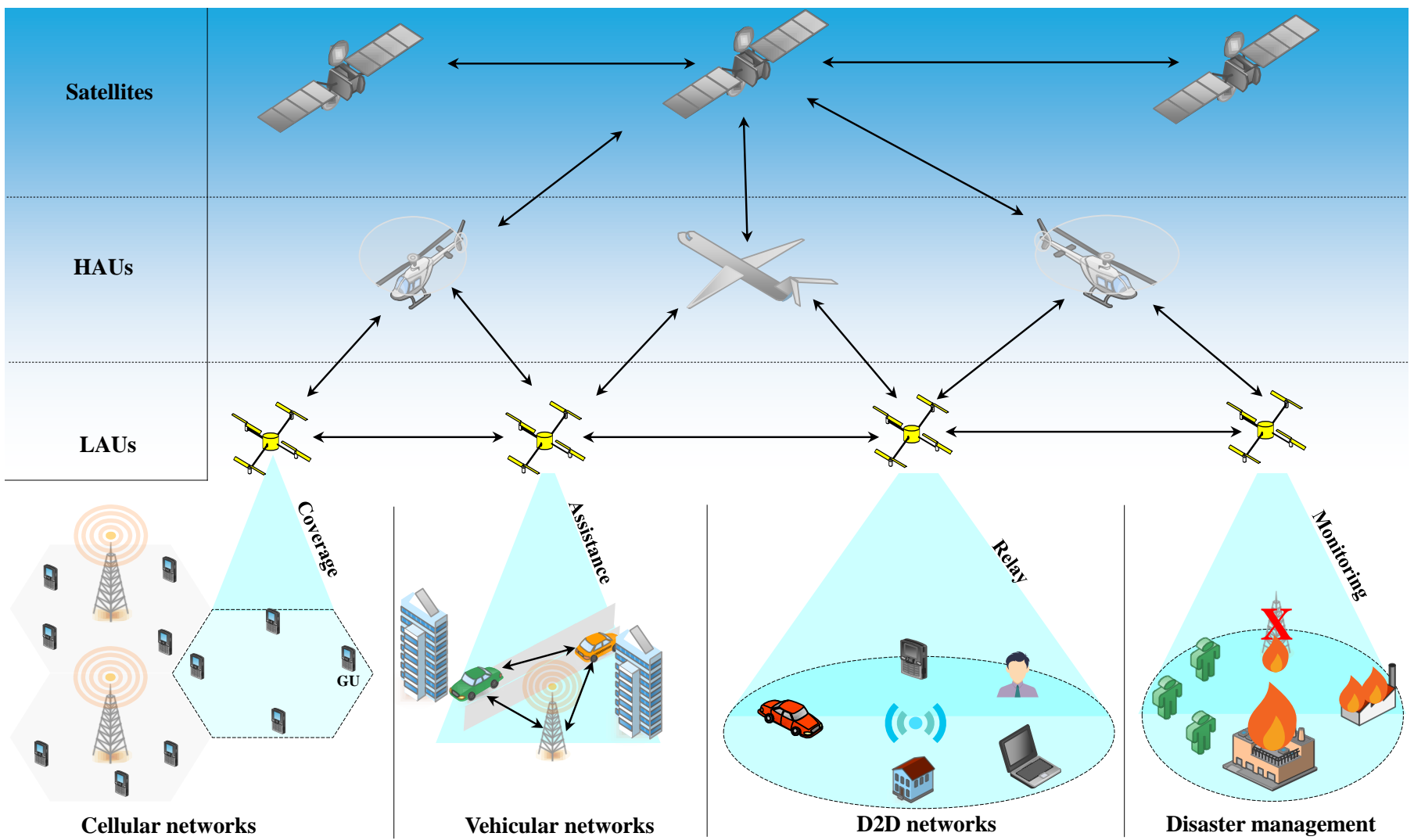

Fig. 2: UAV assistance potential cases.

accurately model A2A channel that takes into consideration the Doppler effect, time-variation of the channel, multi-path fading, antennas' movement, and UAVs' altitude. To build an integrated space-air-ground network, S2A communication is considered as a key component to achieve a more effective UAV-satellite interaction [43]. The S2A channel modeling is generally based on the LoS link where UAVs are able to establish communication (i.e., ensuring the alignment of the spatial beam between UAV and target satellites) with geosynchronous satellites in different orbits during UAV movements [44]. However, as drawbacks, several conditions can directly affect the S2A channel, such as the altitude variation of UAVs and the rain attenuation when using Ka-band and above [45]. To overcome these problems, UAVs are required to continuously modify its beam towards target satellites to maintain the communication link. Consequently, the accurate channel design while considering the aforementioned factors is of great importance, and more importantly when UAVs are deployed to enhance the coverage of terrestrial networks [46], to assist cellular networks [47], and to participate in IoT communications [48]. TABLE II provides a brief comparison between the different channel models described above based on several crucial parameters.

TABLE II: Comparison between A2G, A2A, and S2A links.

\begin{tabular}{|l|l|l|l|}
\cline { 2 - 4 } \multicolumn{1}{c|}{} & Frequency & Scenario/Altitude & $\begin{array}{l}\text { Channel modeling in } \\
\text { the literature }\end{array}$ \\
\hline A2G & $0.7 \mathrm{GHz}-8 \mathrm{GHz}$ & $15 \mathrm{~m}-300 \mathrm{~m}\left(0^{\circ}-90^{\circ}\right)$ & Refs. [42], [49]-[53] \\
\hline A2A & $2.4 \mathrm{GHz}-5.06 \mathrm{GHz}$ & $500 \mathrm{~m}-1500 \mathrm{~m}$ & Refs. [54], [55] \\
\hline S2A & $10 \mathrm{GHz}-50 \mathrm{GHz}$ & $700 \mathrm{~m}-20000 \mathrm{~m}$ & Refs. [56]-[59] \\
\hline
\end{tabular}

\section{B. UAV categorization}

As depicted in Fig. 2, there are three different categories of flying entities that can serve terrestrial networks: (i) LowAltitude UAVs (LAUs), (ii) High-Altitude UAVs (HAUs), and (iii) Satellites. LAUs have a restricted capacity in terms of flight autonomy (i.e., up to 40 minutes of flying time depending on the adopted battery technology), limited geographical area (i.e., restricted mobility pattern), and low payload capacity (i.e., up to 7 kilograms) [60]. Despite their drawbacks, LAUs are considered as a cost-effective and fast-deployment solution for servicing regions where a temporary and additional coverage is required (e.g., concerts, festivals, and sport events), or existing cellular networks are damaged (e.g., natural disasters) [61]. Moreover, LAUs have short-range LoS communication links that are efficiently established to improve the coverage performance [62]. Unlike LAUs, HAUs provide a better performance in terms of flying time and coverage, but their deployment is an expensive solution and may cause an extremely large interference in terrestrial networks [63]. Moreover, HAUs operate at high altitudes in an energy efficient way. Satellites are generally deployed in different orbits, perform complex space-ground tasks, and need pricey and voluminous user equipment. They can be connected with terrestrial networks via wireless communication links [64]. The relationship between these three categories of flying entities can be summarized as follows: 
- Satellites can be considered as centralized controllers and configure and manage the networks of HAUs and LAUs.

- Satellites can also serve as relays for HAUs that in turn can play the role of relays for LAUs.

- All these flying entities maintain robust communication links supporting bandwidth demands and appropriate capacities of the terrestrial first responders.

- HAUs and LAUs can be deployed together with the existing satellites in order to ensure speedy deployment covering larger areas.

Table III presents a brief comparison between these categories of flying entities.

TABLE III: FANET communication comparison.

\begin{tabular}{|l|l|l|l|}
\cline { 2 - 4 } \multicolumn{1}{c|}{} & LAUs & HAUs & Satellites \\
\hline Cost & Cheaper & Expensive & Highly expensive \\
\hline Altitude & Up to $4 \mathrm{~km}$ & Up to $23 \mathrm{~km}$ & Up to $36000 \mathrm{~km}$ \\
\hline Payload & Up to $7 \mathrm{~kg}$ & Up to $1000 \mathrm{~kg}$ & Up to $25000 \mathrm{~kg}$ \\
\hline Coverage & Medium & Large & Huge \\
\hline Endurance & Up to $40 \mathrm{~min}$ & Up to 100 days & Up to 15 years \\
\hline Deployment time & Short-term & Mid-term & Long-term \\
\hline Weight & Up to $10 \mathrm{~kg}$ & Up to $1000 \mathrm{~kg}$ & Up to 450 tons \\
\hline LoS & Low & Medium & High \\
\hline Functionality & Simple & Medium & Complex \\
\hline Energy type & Batteries & Fuel & Hydrazine \\
\hline Flight range & Up to $200 \mathrm{~km}$ & Up to 20 million $\mathrm{km}$ & Undefined \\
\hline Regulations & Safety laws & Global laws & International laws \\
\hline Examples & Quad-copters & Aircraft/Balloons & Geo/Meo/Leo \\
\hline
\end{tabular}

\section{Energy consumption and supply}

Among the most critical issues of UAVs are their restrictedenergy capacity and how to efficiently manage its consumption [65]. The flight duration of UAVs and the lifetime of the whole UAV network are highly impacted by the energy consumption of UAVs, which is related to their mobility pattern, their payloads, their transmission power, and their circuit supply [26]. Most of the UAVs are equipped with rechargeable batteries that can be powered by solar energy, fuels, and other sources of energy. However, it is not conceivable that UAVs frequently return to charge their batteries, which restricts considerably their hovering time [66]. Consequently, the important point to note here is that proper modeling for UAV energy consumption is crucial because the energy consumption of UAVs is not only subjected to circuits, power amplification, and signal processing, but in major part, it is also subjected to additional propulsion energy consumption for remaining in flight. When the communication-related power expenditure is considered to be negligible, several models of propulsion energy consumption have been investigated in the literature. For example, in the works in [67], [68], the energy consumption was modeled based on the acceleration vector and control force, whereas in [69], an empirical energy consumption model was applied for a UAV path planning application that is deployed for aerial imaging where several measurements were performed to investigate the energy consumption of a given UAV with different speeds. In [70], a heuristic model was adopted, which demonstrated that the energy consumption is proportional to the square of the speed of the UAV. In the case when the energy consumption of communication is not negligible, several other measurements are conducted in the literature.
For instance, the energy consumption of an IEEE 802.11n interface under transmission, reception, idle, and sleep modes is $1280 \mathrm{mAh}, 940 \mathrm{mAh}, 820 \mathrm{mAh}$, and $100 \mathrm{mAh}$, respectively [71]. In another example, it was demonstrated that traditional alkaline batteries with a capacity of $860 \mathrm{mAh}$ could provide a flight of 25 minutes under a continuous communication (i.e., reception and transmission) by consuming $740 \mathrm{mAh}$ [72]. As a conclusion to this discussion, the energy consumption modeling is still at the infant stage, and therefore a lot of work should be made to accurately optimize the energy consumption of UAVs. It is worthy to mention here that several crosslayer solutions have been put on the field to improve the energy consumption. Moreover, the emerging topic of energy harvesting to power saving has been extensively studied in recent times. The remaining subsections are dedicated to the energy-aware cross-layer solutions and wireless power transfer concept.

1) Cross-layer energy optimization: To guarantee the energy efficiency of UAV networks, the energy consumption has to be optimized at three different layers: (i) the network layer, (ii) the data link layer, and (iii) the physical layer [73]. In the network layer, there is a crucial need to design energyaware routing protocols to optimize the whole UAV network lifetime where several parameters should be considered, such as the density of UAVs, intermittent connections, scalability, QoS, and overhead. Also, UAVs that have low energy levels have to be spared from any data transmissions and relying only on UAVs with high energy levels [74], [75]. As for the data link layer, the energy-saving can be carried out by listening to eventual traffic, avoiding collisions and overhearing, and enhancing duty cycles. Several MAC protocols are used for energy efficiency, e.g., IEEE 802.11 conserves energy by activating the sleep mode of idle nodes, IEEE $802.11 \mathrm{n}$ conserves energy in multi-poll fashion both in unscheduled and scheduled modes, while IEEE 802.11e conserves energy during the data transmission [76], [77]. At the physical layer, the energy could be saved by optimizing several hardware aspects, such as access to physical medium, power control for the network hardware devices, modulation and demodulation, and encoding-decoding. Moreover, creating new hardware devices that are able of modifying the wireless network, according to the varying traffic demands, shadowing, undefined direction of communication, varying radiation patterns, and interference [78], [79].

2) Wireless Power Transfer (WPT): As an innovative mechanism to provide a perpetual energy supply for limitedpowered UAVs, the WPT concept has been recently adopted as an emergent solution. In fact, WPT is based on the radio frequency (RF) transmission in which dedicated energy receivers (ERs) are deployed in a distributed manner over UAVs to receive RF signals from energy transmitters (ETs). Nevertheless, the robustness and efficiency of WPT are restricted due to the long distances that both often separate ETs and ERs and cause severe propagation losses of RF signals. To address these issues, several works have exploited the adjustable mobility of UAVs in the 3D space [80]-[82]. For instance, it was demonstrated that moving close to the ETs while ensuring LoS links, the efficiency of the WPT is significantly enhanced. 
Moreover, it was also proven that using an omni-directional antenna can maximize the total harvested energy at all ERs. The same conclusion that works have made is that ETs need to be deployed in an ultra-dense manner through fixed locations.

\section{Interference}

The collaboration between UAVs and terrestrial networks faces many challenges that may hinder its progress [83]. The interference is a critical problem that significantly impacts the communication between UAVs and ground entities, which becomes more severe by the dominated-LoS A2G channel when UAVs are hovering at high altitudes [84]. Moreover, the mobility of UAVs can cause a Doppler shift, thus creating severe interference at higher communication frequencies. Also, for the uplink and downlink communications between UAVs and ground BSs, the UAVs may cause interference to adjacent BSs and receive interference from them, respectively [85]. As a result, the problem of interference can be mitigated by diversifying frequency spectrum or by enabling efficient coordination among UAVs, thus requiring a deep study to be put on the field.

\section{E. Mobility planning}

An optimal flight trajectory of UAVs can be defined according to the scenario that they are deployed for. Several constraints have to be considered before defining a planned trajectory, such as the continuous connectivity, the collisions, the energy restriction, and the air corridors [86]. Moreover, some adopted regulations should be taken into account, e.g., avoiding some prohibited zones, such as airports, regional borders, and certain residential areas. To accurately define such trajectories, certain information related to movements of UAVs should be considered, such as the speed and location [87]. As a concrete example, UAVs can be used to carry BSs to serve users located at a particular ground location, and at the same time, UAVs have to reduce their speeds to ensure a good wireless connectivity [88]. In addition, UAVs can also adapt themselves to the users' mobility in a cooperative manner in order to reduce handover, improve energy consumption, and avoid collisions. Consequently, this needs a deep study to be able to define a near-optimal path for UAVs to every situation.

\section{F. $3 D$ beamforming}

To reduce the inter-cellular interference, 3D beamforming can be a suitable solution to support a high number of GUs at different altitudes and elevation angles [89]. Indeed, ground BSs are equipped with directional antennas with transmission power and antenna patterns, which do not allow them to serve all GUs located in a 3D space. As a result, BSs needs to be equipped with the required equipment in order to both easily communicate with hovering UAVs and improve the interference mitigation capacity by using the elevation angles that separate UAVs. The high altitude of UAV-carried flying BSs allows to easily distinguish any GUs regardless of their 3D positions. Moreover, the UAV-to-ground channel characteristics, which provide high LoS communications make UAV-BSs the most appropriate candidates to employ 3D beamforming [90]. It is worthy to note that $3 \mathrm{D}$ beamforming is receiving an outstanding interest in classical cellular networks [91]. This is because it is possible to find two GUs with different elevation angles, thus making 3D beamforming more effective for such kind of networks. Consequently, a lot of efforts should be dedicated to adopting such techniques to improve the performances of different kinds of terrestrial networks.

\section{G. Network security}

A crucial challenge of UAV networks is to ensure their security, as they present a multitude of vulnerabilities, such as malicious attacks, unauthorized intrusion, or even physical attacks [92]. Moreover, the problem of confidentiality is another critical security weakness of such networks, particularly if there is important information to be collected [93]. Indeed, in the case when a given UAV is diverted or has its control stolen, it can probe critical information from the authenticated network by using it as a gateway. All these challenges are still an outstanding problem and can be explored more deeply.

\section{H. Payload}

Payload defines the maximum amount of weight that a UAV can transport, which has a direct effect on the endurance capabilities, communication, coverage, and an operational altitude of the UAV [94]. The more payload that a UAV can carry, the more accessories can be carried. These accessories can be in the form of sensors for reconnaissance, video cameras for monitoring, mobile phones for assisting cellular communications, or even BSs for providing cellular services [95]. Consequently, the payload of UAVs, and especially LAUs should be investigated in accordance with many issues that may come up.

\section{Altitude}

The operational altitude of UAVs can be determined according to the environment where they are deployed. Indeed, the altitude of UAVs in an urban area comprising high-rise buildings has to be higher than that of UAVs deployed in suburban environments [96]. This is because GUs require higher LoS connectivity and path-loss reduction while minimizing shadowing and reflection phenomenons. The altitude can be also determined based on the number of existing UAVs with the aim to maximize the coverage of ground networks or to achieve a given task [97]. As a result, attention should be focused on the definition of the appropriate altitudes of UAVs.

\section{J. Aerial (UAV) User Equipment (UAV-UE)}

UAVs can be deployed in a variety of applications ranging from surveillance [98], remote sensing [99], package delivery [100], and virtual reality applications [101]. Indeed, in most cases, UAVs are acting as UAV-user equipment (UAV-UE) demonstrating their capacity to flexibly move and optimize their trajectory to rapidly achieve their tasks. Often, UAV-UEs are required to establish $\mathrm{LoS}$ connectivity to terrestrial BSs 
to guarantee a certain high-speed data access while avoiding inter-cell interference that can occur. Moreover, different challenges that face UAV-UEs need to be considered, such as their restricted-energy capacity, their communication channel conditions with ground BSs, and their continuous and high mobility introducing new design considerations.

\section{K. Aerial (UAV) Base Station (UAV-BS)}

UAVs are envisioned to be used as flying aerial BSs or UAVBSs to both provide cost-effective wireless communications to selected areas and support the connectivity of terrestrial wireless networks [102]. Indeed, UAV-BSs have the ability to adjust their altitudes and movements, avoid obstructions, and always look for establishing LoS communication with GUs. Different kinds of UAVs can be adopted as UAV-BSs, such as LAU-BSs for providing short-term connectivity to given geographical areas or temporary events (e.g., stadium, festivals, concert) and HAU-BSs for ensuring long-term coverage to a specific zone (e.g., rural environments).

\section{UAV-UGV COORDINATION}

The cooperation between multiple unmanned systems has recently attracted the attention of the scientific community worldwide and developers. Indeed, this kind of systems is equipped with special sensors, communication systems, data processing units, and automatic control, with the aim to autonomously perform tasks without human interaction [103]. In this section, we are interested in the cooperation between UAVs and Unmanned Ground Vehicles (UGVs) in order to exploit the advantages provided by each type of nodes and overcome the specific constraints of the others during the accomplishment of a specific task. Nevertheless, some challenges are worth mentioning, as follows:

- Addressing the energy constraint of UAVs by using UGVs as energy sources, and vice-versa.

- Guiding UGVs between target points using UAVs.

- Tracking mobile targets by UAVs while considering their surroundings.

- Adjusting the mobility of UAVs according to the movements of UGVs.

The following subsections discuss the major contributions proposed in this field and Table IV portrays a summary of these UAV-UGV contributions.

\section{A. Path Planning}

As illustrated in Fig. 3, when UGVs are deployed in complex or hostile environments, it will be extremely difficult to progress normally. Therefore, the hovering of one or multiple UAVs above UGVs and overseeing their surroundings can help them to both find the optimal path between two points and guide them across the path [104]. In the case when UAVs want to track a given object (e.g., tracking a UGV for a safe landing), they estimate the trajectory of this object based on the intercepted images while it is moving [105]. We discussed the major contributions adopting this kind of techniques.
UAV-UGV coordination as a new network architecture is becoming increasingly popular, which paved the path for the emergence of an important number of applications. To elaborate, Ropero et al. [106] proposed a hybrid cooperative system composed of both UGV and UAV. The idea behind this system is to allow UGV and UAV to reach all target points by considering UGV as a moving recharging station to address the restricted-energy capacity of the UAV. As for the UAV, it is used as a system to both help reaching the target points and address the UGV functionality constraints. Moreover, to optimize the traveling distance, a strategy combining combinatorial classic techniques and modern evolutionary approaches is formulated. However, the environmental constraints are neglected, which is not realistic and can disturb the right functionality of this scheme. To overcome this problem, Lakas et al. [107] proposed an approach considering the existing ground obstacles. In fact, it is based on a UAV incorporating a camera and collecting images of the surrounding areas with the aim to assist the UGV to reach its final destination while avoiding existing obstructions on the ground. Moreover, a visionbased algorithm is used to recognize passages, obstacles, and roads, in order to compute a near-optimal path over them leading the UGV to its final destination. At the same time, the UAV follows the progress of the ground vehicle towards the destination by monitoring and following its movement. The trajectory generation is based on an improved version of the $\mathrm{A}^{*}$ algorithm [108]. However, applying such a technique requires high processing by the $\mathrm{UAV}$, and thus consuming more energy.

To both reduce the energy consumption and perform efficient cooperation between UAVs and UGVs, Arbanas et al. [109] proposed a distributed system of control dedicated to complex missions. Two kinds of controls are considered by this system: (i) Low-level control and (ii) High-level control. The low-level control includes several tasks, such as mapping, path planning for both UGVs and UAVs, landing UAVs onto UGVs, and picking up lightweight UGVs using UAVs. The high-level control includes distributed ad hoc tasks in order to achieve the missions and the communications between UAVs and UGVs. To provide an accurate ground map, Li et al. [110] proposed a path planning scheme using UAV-UGV cooperation. UAVs are deployed to identify obstructions on the ground using vision sensors in order to build a map. The latter is used for path planning of UGVs. However, the authors supposed that UAVs are flying at the same altitude. To track a moving target, Yu et al. [111] proposed a cooperative path planning based on both UAVs and UGVs. The purpose of this work is to track mobile targets in urban areas and to evaluate the performances of the proposed algorithm by varying the degree of occlusions. The different states of the target are modeled using a dynamic occupancy grid updated periodically by the Bayesian filter. However, the communications between UGVs are omitted, which can enhance the performance of the proposed scheme.

Findings: As discussed in this subsection, it is evident that path planning is a promising technique for efficiently accomplishing UAV-UGV coordination towards several kinds of missions. Consequently, we summarize the lessons learned 


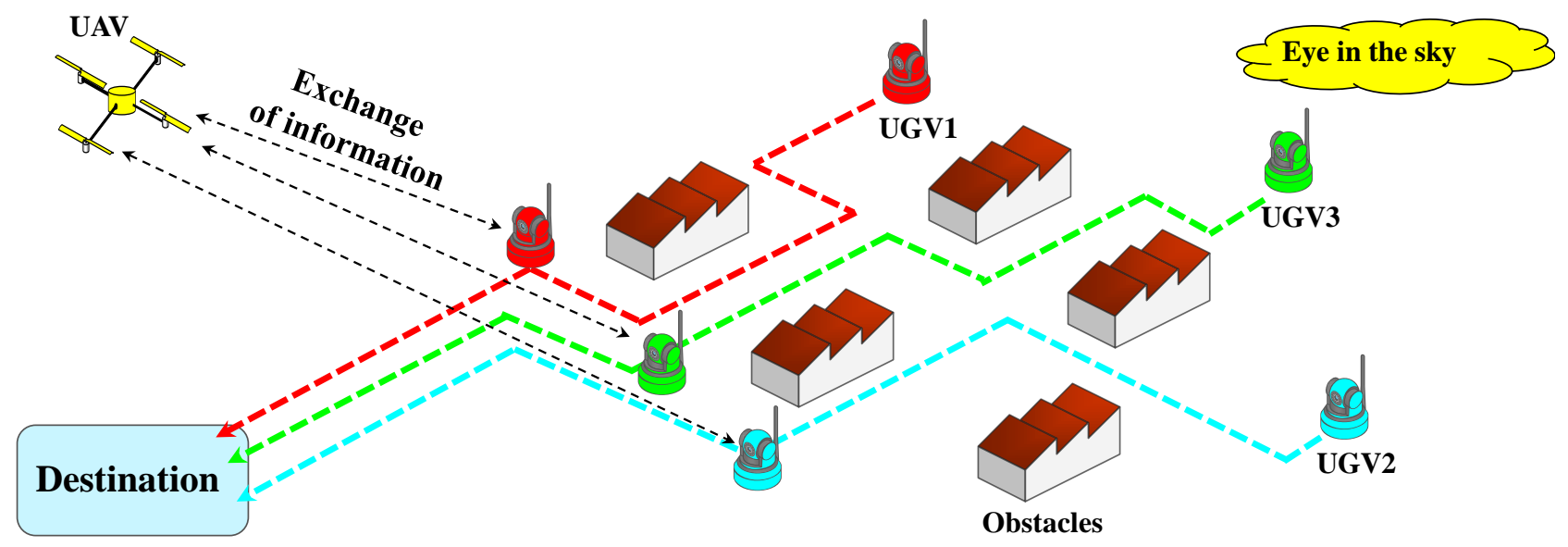

Fig. 3: UAV-UGV path planning.

as follows:

- The cooperation between UAVs and UGVs can perform several kinds of missions and improve the performances of the whole system. For instance, UAVs can guide UGVs to both avoid obstructions and reach their final destinations. Also, UGVs can be considered as moving recharge stations helping UAVs to surpass their energy restriction.

- The majority of path planning contributions neglects the existing obstructions and the limited energy capacity of both UAVs and UGVs.

- The mobility of UAVs and UGVs is partially investigated. Moreover, the exchange of information between each other is omitted in the majority of cases.

\section{B. Intelligence Surveillance and Reconnaissance}

To avoid exposing humans to danger, Intelligence, Surveillance, and Reconnaissance (ISR) missions are exploited to perform coordinated acquisition of accurate information in real-time in order to support the activities of a human operator [112]. Indeed, ISR missions can involve UAVs and UGVs cooperating with each other to obtain situational awareness with the aim to accomplish the tasks in an efficient way (c.f., Fig. 4) [113]. In the following, five major contributions proposed in the literature are discussed.

A pioneering work is proposed by Tokekar et al. [114] in which a system based on UAV-UGV coordination tries to gather information about nitrogen level over a farm with the aim to enhance a fertilizer application. In this scenario, the UGV can carry the UAV that has to take aerial images of many points of interest such that the UAV does not consume any energy. Moreover, the UGV is able to pick up the UAV over any geographical position and at the appropriate time based solely on its speed, its direction, and its location. This work has shown that it cannot be adapted with multiple UAVs and UGVs. To take into account this case, Ghamry et al. [115] proposed a cooperation strategy between multiple UAVs and UGVs for forest surveillance and fire detection. This strategy uses leader-following formation algorithms applied in both UAVs and UGVs. Indeed, the UGVs are exploited to carry the UAVs to the area of interest, in which UAVs take-off and begin the surveillance mission. In the case when a UAV detects a fire, it transmits the location of the fire to the leader UGV that will generate the reference path to be followed for UAVs in order to continuously monitor the spread of fire. It was shown that in the case of communication loss between UAVs, this system can be out of service. To monitor operations in humanitarian demining, in [116], the UGV is equipped with a vision system with the aim to detect the hovering UAV based on its high brightness LED. This information is exploited to send different commands to the UAV. This strategy allows the UAV to autonomously follow the UGV using a vision tracking algorithm. However, the energy consumption of both UGV and UAV are not considered by this system. Another interesting UAV-UGV cooperation is proposed in [117] where a set of UGVs equipped with sensors follow a preplanned path and scan the points of interest. Equipped with a system for visual relative localization and a bottom camera, UAVs are launched to perform the inspection only when it is impossible to reach a given location of interest by UGVs. However, the specific use case of this system can be considered as its main drawback. To supervise wildfire fighting, the work in [118] proposed a cooperative framework for hierarchical UAV-UGV coordination. This work is adapted for wildfire detection and fighting wherein the topmost level, an airship is used as a centralized controller to carry out mission planning and task assignment for UAVs and UGVs. Nevertheless, an unrealistic assumption is considered in which the airship is supposed to be always operational, which is not reasonable under certain conditions.

Findings: The key lessons learned from this subsection are listed below:

- UAVs and UGVs can significantly improve the completion time of a given mission if they are efficiently exploited and coordinated with each other.

- The task assignment for UAVs and UGVs needs to be carefully studied to accomplish a given mission in an efficient and accurate way.

- The deployment of a robust communication strategy between UAVs and UGVs is of major concerns. 


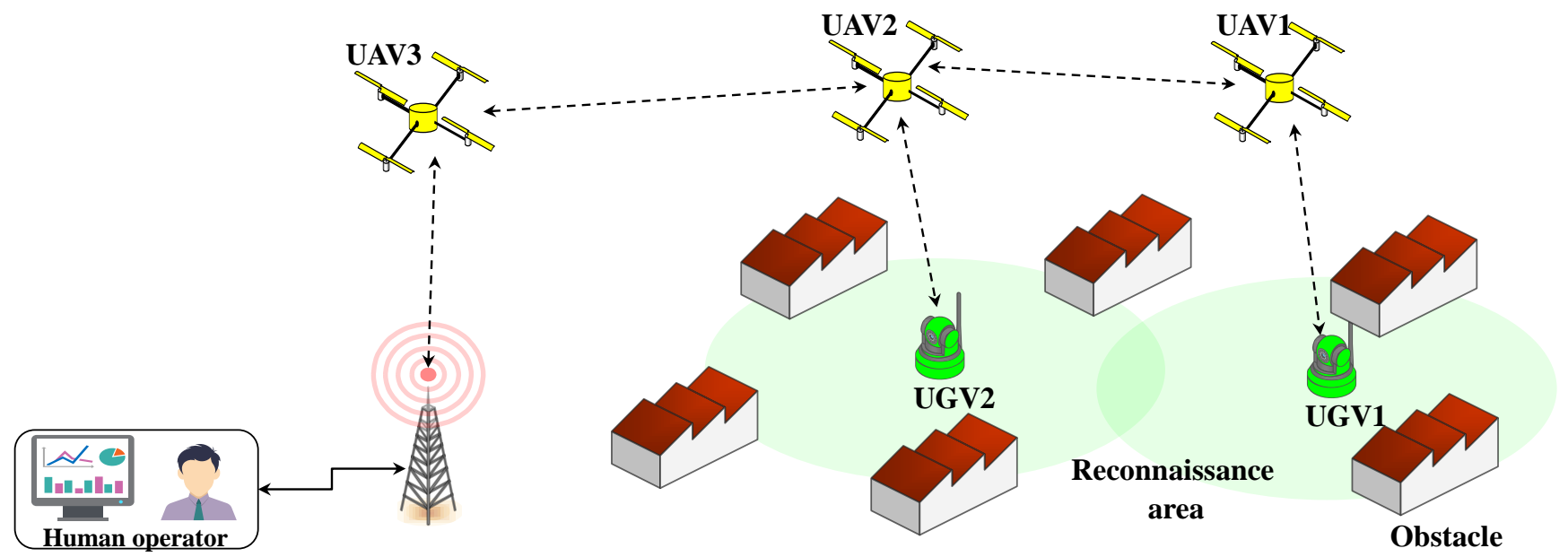

Fig. 4: UAV-assisted intelligence surveillance and reconnaissance.

\section{Formation Control}

The use of UAVs and UGVs creates a coordinated group regarding the missions that they plan to accomplish in a timely way [119]. Nevertheless, this kind of collaboration involving different dynamic mobility models has many challenges in communications and formation control [120]. To address these issues, different techniques have been adopted to keep the members of the coordinated group within a certain geometric form (c.f., Fig. 5). In the following, we provide the major contributions that have been made in this way.

Sometimes, the mobility of both UAVs and UGVs needs to be formed and controlled with the aim to perform a given mission in a timely manner. For instance, to track and control crowds, Minaeian et al. [121] proposed an algorithm combining both a GIS localization and vision-based crowd detection for a cooperative UAV and a set of UGVs. The key idea behind this work is to use UGVs to convert the detected target locations into their GIS coordinates. This work has proven its efficiency when using a group of UGVs rather than a single UAV. However, localization can be further enhanced. Similarly, the authors of [122] proposed coordination between a swarm of UGVs with a single UAV. The UGVs are organized based on different functions that are designed around the location of the UAV. This allows UGVs to travel on the constructed surface that controls the overall geometry of the group and the spacing of individual UGVs. It was shown that this system is able to function only under a small number of UGVs. Brandao et al. [123] proposed a distributed control scheme based on a leader-follower concept comprising a single UAV and a set of UGVs. The objective of the scheme is to allow the UAV to perform the mission of tracking the current centroid of the ground formation. However, the ground formation tracking can fail in case of obstacle avoidance. To handle this issue, Barnes et al. [124] proposed a coordinating and controlling strategy of hybrid unmanned systems moving as a swarm. In this strategy, one or more UAVs are used to coordinate groups of UGVs in which UAVs can guide the group of the UGVs to control their overall formation, and vice versa. As a drawback, this scheme did not consider the energy consumption and external conditions during the swarm formation.

Findings: In summary, the main lessons learned from this subsection include:

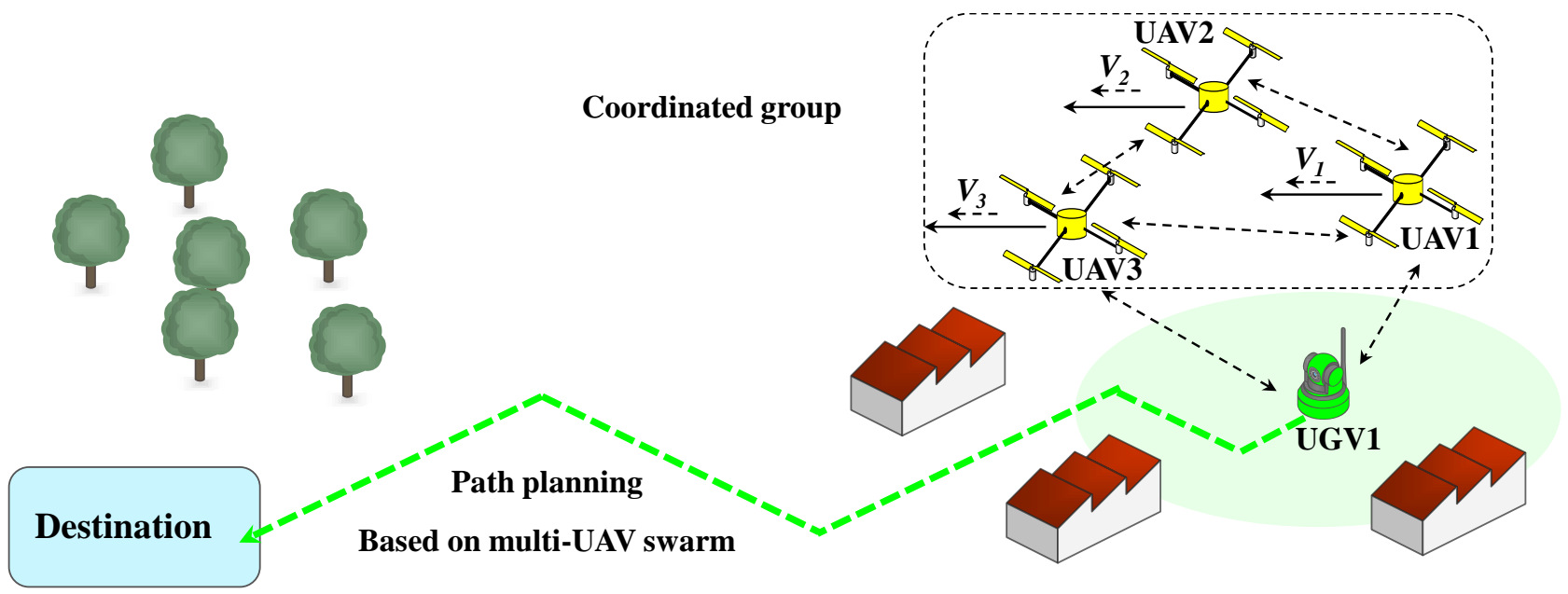

Fig. 5: UAV-assisted formation control. 
TABLE IV: Summary of contributions to UAV-UGV coordination.

\begin{tabular}{|c|c|c|c|c|c|c|c|}
\hline & & $\begin{array}{l}\text { Density and Mobility of } \\
\text { UAVs }\end{array}$ & $\begin{array}{c}\text { Types of ground } \\
\text { nodes }\end{array}$ & $\begin{array}{l}\text { Deployment } \\
\text { environment }\end{array}$ & Objective & Advantage & Drawback \\
\hline \multirow{5}{*}{ Path planning } & Ref. [106] & Single UAV/Mobile & Single UGV & Exploration area & $\begin{array}{l}\text { Overcoming the energy constraint of } \\
\text { the UAV by considering the UGV as a } \\
\text { recharge station following it. }\end{array}$ & $\begin{array}{l}\text { Generated a near optimal coordinated } \\
\text { path plan to reach all targets. }\end{array}$ & $\begin{array}{l}\text { Did not consider weather conditions } \\
\text { and environmental constraints. }\end{array}$ \\
\hline & Ref. [107] & Single UAV/Mobile & Single UGV & Post-disaster area & $\begin{array}{l}\text { Assisting the UGV by cooperatively } \\
\text { plan its trajectories to reach the target } \\
\text { destination }\end{array}$ & $\begin{array}{l}\text { Successfully mapped the explored area } \\
\text { for the UGV by using multiple } \\
\text { techniques. }\end{array}$ & $\begin{array}{l}\text { A high processing is required in the } \\
\text { UAV, thus consuming more energy. }\end{array}$ \\
\hline & Ref. [109] & Single UAV/Mobile & Single UGV & $\begin{array}{c}\text { Unknown } \\
\text { environment }\end{array}$ & $\begin{array}{l}\text { Using UAV to provide obstacle-free } \\
\text { trajectories for the UGV. }\end{array}$ & $\begin{array}{l}\text { Enhancing energy consumption by } \\
\text { using an efficient planning method. }\end{array}$ & $\begin{array}{l}\text { Did not support multiple UAVs and } \\
\text { multiple UGVs during the planning } \\
\text { path. }\end{array}$ \\
\hline & Ref. [110] & Single UAV/Mobile & Multiple UGVs & Disaster area & $\begin{array}{l}\text { Providing an accurate ground map to } \\
\text { obtain an efficient path planning for } \\
\text { UGVs }\end{array}$ & $\begin{array}{l}\text { Obtained a less costly path for UGVs } \\
\text { compared with traditional techniques. }\end{array}$ & $\begin{array}{l}\text { Considered only 2D trajectories of } \\
\text { UAVs. }\end{array}$ \\
\hline & Ref. [111] & Multiple UAVs/Mobile & Multiple UGVs & Urban area & $\begin{array}{l}\text { Tracking a moving target using a } \\
\text { cooperative path planning }\end{array}$ & $\begin{array}{l}\text { Designed efficient methods to track } \\
\text { different movements of a specific } \\
\text { target. }\end{array}$ & $\begin{array}{l}\text { Did not consider the communications } \\
\text { between UGVs. }\end{array}$ \\
\hline \multirow{5}{*}{ ISR } & Ref. [114] & Single UAV/Mobile & Single UGV & Agriculture area & $\begin{array}{l}\text { Predicting the status of crops by using } \\
\text { intercepted data from aerial and } \\
\text { ground nodes }\end{array}$ & $\begin{array}{l}\text { Maximized the number of visited } \\
\text { points by the UAV while considering } \\
\text { its restricted-energy capacity. }\end{array}$ & $\begin{array}{l}\text { Neglected the case of multiple UGVs } \\
\text { and UAVs. }\end{array}$ \\
\hline & Ref. [115] & Multiple UAVs/Mobile & Multiple UGVs & Forest area & Detecting fire & $\begin{array}{l}\text { UAVs communicated with the UGV } \\
\text { leader to minimize their consumed } \\
\text { energy. }\end{array}$ & $\begin{array}{l}\text { Did not handle connectivity between } \\
\text { UAVs in the case of detecting fire. }\end{array}$ \\
\hline & Ref. [116] & Single UAV/Mobile & Single UGV & Mined area & $\begin{array}{l}\text { Monitoring operations in humanitarian } \\
\text { demining }\end{array}$ & $\begin{array}{l}\text { Successfully tracked the UAV under } \\
\text { different conditions. }\end{array}$ & $\begin{array}{l}\text { No measures taken to allow the UAV } \\
\text { to land on the UGV (e.g., to recharge } \\
\text { its battery). }\end{array}$ \\
\hline & Ref. [117] & Single UAV/Mobile & Single UGV & Indoor environment & $\begin{array}{l}\text { Periodical monitoring of an indoor } \\
\text { environment }\end{array}$ & $\begin{array}{l}\text { Proposed two techniques of landing } \\
\text { system and active helipad, which } \\
\text { provided good performances. }\end{array}$ & $\begin{array}{l}\text { Cannot be adapted to inhospitable } \\
\text { areas that are exposed to different } \\
\text { constraints. }\end{array}$ \\
\hline & Ref. [118] & $\begin{array}{l}\text { Multiple UAVs/Airship } \\
\text { Mobile/Static }\end{array}$ & Multiple UGVs & High-risk areas & $\begin{array}{l}\text { Wildfire fighting using a hierarchical } \\
\text { UAV/UGV platform }\end{array}$ & $\begin{array}{l}\text { Designed a near optimal mission and } \\
\text { coordinated the tasks of each UGV in } \\
\text { an efficient manner. }\end{array}$ & $\begin{array}{l}\text { Assumed that the airship is always } \\
\text { operational, which is not reasonable } \\
\text { under certain conditions. }\end{array}$ \\
\hline \multirow[b]{4}{*}{ Formation control } & Ref. [121] & Single UAV/Mobile & Multiple UGVs & Border area & Tracking and controlling crowds & $\begin{array}{l}\text { Efficiently detected motion for UAV, } \\
\text { human for UGV, and real world } \\
\text { localization using UGVs. }\end{array}$ & $\begin{array}{l}\text { The landmark assignment method } \\
\text { needs to be enhanced for a robust } \\
\text { localization. }\end{array}$ \\
\hline & Ref. [122] & Single UAV/Mobile & Multiple UGVs & $\begin{array}{c}\text { Outdoor } \\
\text { environment }\end{array}$ & $\begin{array}{l}\text { Guiding a swarm of the UGVs to } \\
\text { control their overall formation using a } \\
\text { UAV as a leader robot }\end{array}$ & $\begin{array}{l}\text { Demonstrated the effectiveness of the } \\
\text { adopted swarm function. }\end{array}$ & $\begin{array}{l}\text { The precision of this strategy is only } \\
\text { demonstrated with a small number of } \\
\text { UGVs. }\end{array}$ \\
\hline & Ref. [123] & Single UAV/Mobile & Multiple UGVs & Large area & $\begin{array}{l}\text { Tracking the centroid of UGVs' } \\
\text { formation }\end{array}$ & $\begin{array}{l}\text { The ground formation is followed } \\
\text { using only the vision system as an } \\
\text { exteroceptive and interoceptive sensor. }\end{array}$ & $\begin{array}{l}\text { Did not consider the loss of ground } \\
\text { formation tracking during obstacle } \\
\text { avoidance. }\end{array}$ \\
\hline & Ref. [124] & Multiple UAVs/Mobile & Multiple UGVs & $\begin{array}{l}\text { Outdoor } \\
\text { environment }\end{array}$ & $\begin{array}{l}\text { Protecting a convoy of UGVs using a } \\
\text { UAV swarm }\end{array}$ & $\begin{array}{l}\text { Several kinds of functions are used for } \\
\text { controlling UAV and UGV swarm } \\
\text { formation, obstacle avoidance, and the } \\
\text { overall swarm movement. }\end{array}$ & $\begin{array}{l}\text { Did consider environmental parameters } \\
\text { during the swarm formation. }\end{array}$ \\
\hline
\end{tabular}

- To keep a set of coordinated UGVs within the same group, UAVs can act as flying references. Indeed, a selected UAV can freely move around a given area while controlling the movements of a group of UGVs. Such coordination requires reliable communications between UGVs and the UAV.

- Different obstructions can distort the formation control of UGVs, and especially when no planning path is made beforehand.

- Different selection methods need to be carefully investigated in order to select the appropriate UAVs and UGVs that are responsible for their respective control formation.

\section{Open Research Challenges}

To coordinate UAVs and UGVs, several optimization problems raised during the definition of control inputs for this unmanned system. Indeed, the control inputs need to be organized, processed, and shared, in order to optimally achieve the goals in a timely and accurate way according to the task goal. Therefore, the optimization problems can be classified into three categories: (i) path planning, (ii) monitoring and reconnaissance, and (iii) formation control. For instance, in the first category, the ignorance of several factors is distinguished, such as the surrounding environment, high processing consuming more energy for unmanned vehicles, the definition of the required number of UAVs and UGVs, the definition of optimal altitude of UAVs, and sometimes the communication between UGVs is omitted. In the second and the third categories, inhospitable areas, a robust localization, and the loss of ground and aerial formation tracking. Consequently, the aforementioned challenges need to be deeply investigated and explored.

\section{UAV ROUTING}

During the last decade, the assistance of terrestrial networks by UAVs has attracted increased attention from researchers [125]. Indeed, the use of UAVs has presented a flexible capability to be deployed to assist terrestrial networks when they suffer from poor connectivity [126]. Also, UAVs can both reach and cover areas that are inaccessible by terrestrial nodes [127]. However, this kind of UAV assistance application presents important challenges for researchers to consider as follows:

- Overcoming the frequent fragmentation of vehicular networks by using UAVs as communication bridges.

- Placing UAVs at the appropriate places to efficiently serve communicating vehicles.

- Finding immediately alternative solutions based on UAVs when the network on the ground disconnects occasionally.

- Overcoming the restricted-energy capacity of UAVs while serving terrestrial networks.

In this context, several UAV-assisted routing solutions have been proposed in the literature, and especially those supporting VANETs and other kinds of terrestrial networks. In this section, we describe the most relevant UAV-assisted routing solutions for VANETs and ground networks. Table V shows a summary of the existing major routing contributions using UAVs to improve the routing process of terrestrial networks.

\section{A. VANET}

Being characterized by the high mobility, frequent topology changes, and recurring fragmentation, VANETs provide multiple complex challenges to data routing [128]-[130]. To handle these issues, UAVs can be quickly deployed to assist VANETs as relays and forward data packets among vehicles 


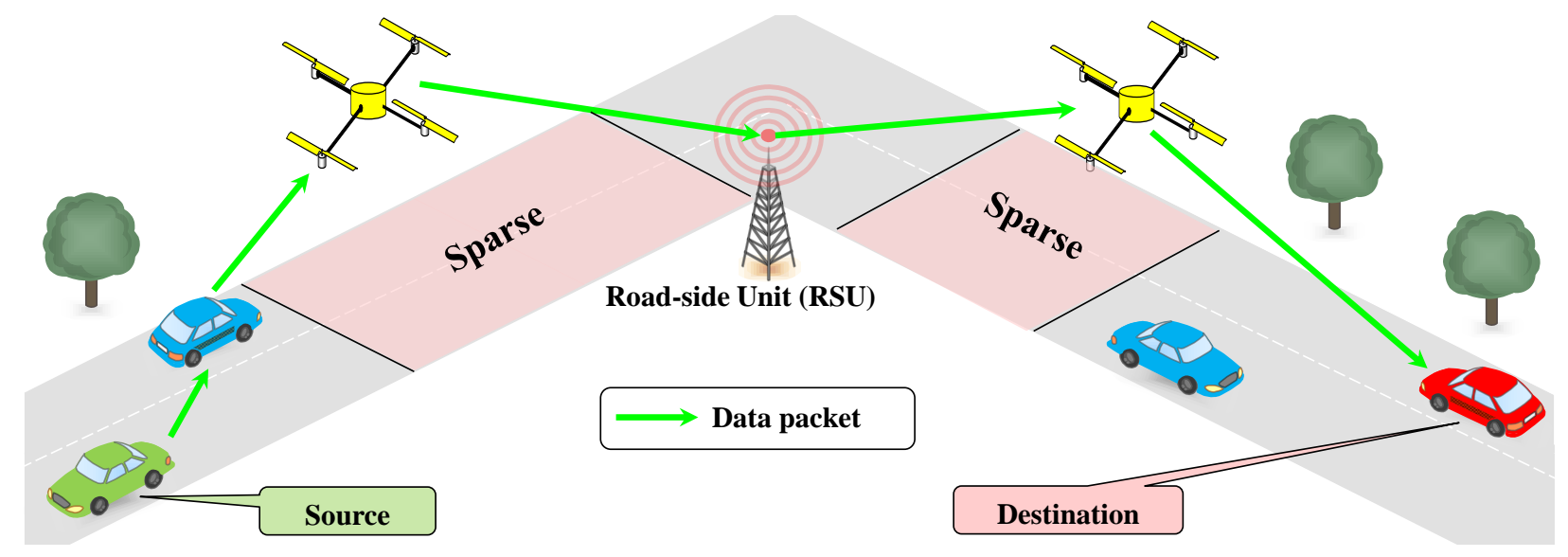

Fig. 6: UAV-assisted routing in VANETs.

when the communication between vehicles on the ground is not possible [131], as shown in Fig. 6. Moreover, UAVs can provide reliable LoS links with ground vehicles, which makes them a suitable option to serve VANETs.

Deploying UAVs over terrestrial networks, particularly VANETs, is becoming increasingly common to overcome the limitations of communications between vehicles. For instance, the authors of [132] proposed a reactive routing protocol dedicated to VANETs considering four separate phases to establish communication between two communicating nodes. Firstly, a discovery process is used (i.e., broadcasting a route request (RREQ) packet) to discover all possible paths based on static size routing packets in order to limit the overhead. Secondly, a decision process is taken to select the most appropriate routing path based on its degree of connectedness and unicastly send a route reply (RREP) packet back to the source. Thirdly, a data delivery process based on several techniques is used to reliably transmit data packets to their right destinations. Finally, a maintenance strategy is used to recover suddenly disconnected paths by finding alternative solutions. However, the adopted discovery strategy may generate a high overhead, and especially in the high density of vehicles. In the same way, the work in [133] proposed flooding technique that instantly respond at each disconnection occurring in the network while avoiding existing obstructions. Indeed, a set of UAVs are deployed to play the role of alternative solutions in the case when there is no connected routing path on the ground between the communicating nodes. In addition, the routing paths are established on the basis of the longevity and regulation of each path using the expiration time and the amount of traffic, respectively. Nevertheless, the adjustable mobility of UAVs could be further optimized to place them at the appropriate locations according to the ground disconnections. Another strategy is adopted by the work proposed in [134]. Indeed, a UAV-assisted routing solution is proposed for VANETs with the aim to maximize the throughput under delay constraints. This maximization strategy is performed in order to find an optimal delivery strategy for data dissemination by improving the transmission rate. Moreover, a polynomial time approximation technique is used in conjunction with the latter strategy to get at an approximate solution. This network is modeled as an edge-weighted graph where the vertices are a set of vehicles and UAVs and the edges are a set of weighted edges that indicate the transmission rate and the transmission delay of each edge. The optimal path is the one that has a maximum transmission rate and a restricted transmission delay.

As a further advance, UAVs can be also used as Store-Carry and Forward (SCF) nodes to assist ground vehicular networks in order to improve the connectivity and that in the presence of non-cooperative vehicles. For instance, the work in [135] developed a mathematical model in order to analyze the impact of non-cooperative vehicles over the connectivity performance of a VANET. Indeed, when vehicles are grouped into multiple disconnected clusters. Data packets cannot transit to another cluster until their carrying vehicles become members of that cluster, and especially when there are non-cooperative vehicles between these two clusters. In the contrast of [135], the work presented in [136] studied the impact of deploying UAVs in the sky to assist a fully-collaborative VANET on the ground. For this purpose, several mathematical models are developed in order to reduce the dependency on the density and connectivity of ground vehicles. In this work, there are two different sets of UAVs flying in the two directions of the road, respectively. This guarantees that at any time, there will be $k$ UAVs flying from a source vehicle to a target destination, which can act as SCF nodes for the ground vehicles moving in that same direction. To avoid the high delay introduced by the use of the SCF strategy, the work proposed in [137] considered two routing components when communication needs to be established between a pair of source and destination nodes. The first component is applied to the ground between vehicles while the second one is applied in the sky between UAVs. These simultaneous executions are performed to find the most appropriate way to deliver data packets. For instance, when a source vehicle wants to send a data packet to a destination vehicle, a reactive strategy is initiated in the sky and a connectivity-based routing strategy is performed on the ground. In this protocol, the routing path in the sky is always favored if, and only if, it is successfully established because it is rarely confronted with the problem of network fragmentation. 
Findings: There are many fundamental lessons learned as follows:

- Self-organizing and flexible UAVs can be full members of existing VANETs and participate as relays during the routing process. In the case of intermittent connectivity, UAVs can also play the role of SCF nodes to bridge communication gaps between isolated communicating vehicles.

- Despite promising use cases (i.e., the different adopted techniques to enhance routing) of UAVs in VANETs, a number of design challenges are distinguished in each use case. Indeed, when UAVs act as relays on top of VANETs, many challenges, such as reducing the induced overhead, regulating the energy consumption among UAVs, and defining the appropriate mobility models, need to be solved.

- UAV-VANET communications are impacted by air-toground and air-to-air communications. Thus, channel modeling in UAV-assisted VANETs is an important challenge and can be made using several techniques available across the literature [53], [138]-[140].

\section{B. Ground Networks}

The flexibility of UAV deployment can be a promising factor to serve different ground entities. For instance, UAVs can be used as a service recovery after a ground BS damage in order to provide the required coverage [141]. Also, UAV communications exhibit important features that can enhance ground network performance and applications. Indeed, embedded with wireless interfaces and navigation equipment, it is very easy to organize UAVs in an ad hoc network or multiUAV network communicating with each other and assisting ground networks. For instance, UAVs have the ability to carry or relay packets between isolated ground clusters comprising ground nodes (i.e., sensors, laptops, smart-phones, vehicles, etc.), thus playing the role of a bridge between them, as illustrated in Fig. 7.

To expound a litter further, the idea of deploying UAVs over a terrestrial network was put forth, e.g., by the authors of
[142] where they proposed a UAV-assisted routing protocol for WSNs. This routing strategy assumes that the ground WSN is organized in the form of clusters and adopts the SCF strategy to collect information from ground sensor nodes. Moreover, several performances have been improved, such as the network lifetime and energy efficiency. However, the authors assume that only static data traffic loads are considered during data gathering. In the same direction, the work in [143] exploited multiple UAVs as a flying relay network in order to recover the communication gap between isolated ground sensors and a BS located further away. An algorithm is proposed to maintain the connectivity of the links, to optimally deploy UAVs, to reduce the packet losses, and to ensure the data delivery to destinations. An unrealistic assumption is made by the authors in which they supposed that the UAV network is considered to be connected all time. As for disaster management and coverage recovery scenarios, in [144], a routing strategy is adopted to make a trade-off between a robust relay technique and an energy-efficient data collection from sensors to the BS. It was shown that authors cannot provide a routing hole repair occurred in UAV networks. When it is a question to support delay-insensitive applications, the work in [145] combined two different routing techniques: (i) a DTN routing in the sky between UAVs and (ii) the traditional routing protocol Ad hoc On-Demand Distance Vector (AODV) [146] on the ground. The idea behind this work is to compensate for the poor connectivity of the network by using UAVs as SCF nodes to transmit the messages between a pair of ground source and destination. It was distinguished that a high delay is introduced by the excessive use of the SCF technique. To avoid the bandwidth restriction, the work in [147], proposed a hierarchical routing protocol, which considers a three-level network structure comprising a ground level network, a mobile backbone network, and UAV network flying at high altitudes. This conception allows relaying information between ground entities, to provide point-to-point wireless links, and to ensure a successful data transmission between any pair of backbone nodes. However, the complexity of this architecture required high processing and a high amount of message exchange.

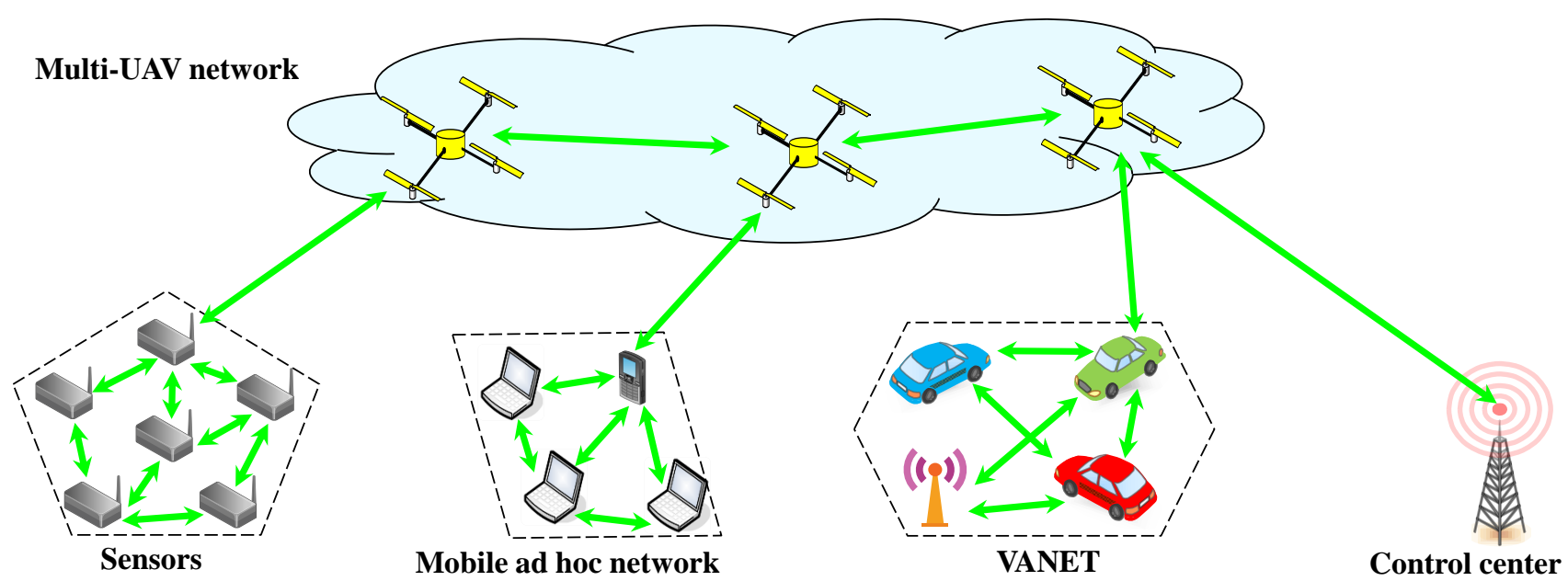

Fig. 7: UAV-assisted routing in ground networks. 
TABLE V: Summary of contributions to UAV-assisted routing.

\begin{tabular}{|c|c|c|c|c|c|c|c|}
\hline & & $\begin{array}{l}\text { Density and Mobility of } \\
\text { UAVs }\end{array}$ & $\begin{array}{l}\text { Types of ground } \\
\text { nodes }\end{array}$ & $\begin{array}{l}\text { Deployment } \\
\text { environment }\end{array}$ & Objective & Advantage & Drawback \\
\hline \multirow{6}{*}{ VANET } & Ref. [132] & Multiple UAVs/Mobile & $\begin{array}{c}\text { Hybrid } \\
\text { (Vehicles/RSUs) }\end{array}$ & Urban & Optimizing VANET routing process & $\begin{array}{l}\text { Increased both the delivery ratio and } \\
\text { alternatives in case of disconnections }\end{array}$ & $\begin{array}{l}\text { Introduced more overhead during the } \\
\text { discovery process, and especially in } \\
\text { high densities. }\end{array}$ \\
\hline & Ref. [133] & Multiple UAVs/Mobile & $\begin{array}{c}\text { Hybrid } \\
\text { (Vehicles/RSUs) }\end{array}$ & Urban & $\begin{array}{l}\text { Increasing the number of alternative } \\
\text { solutions in case of disconnections }\end{array}$ & $\begin{array}{l}\text { Maximized the number of alternative } \\
\text { solutions, and thus the delivery ratio }\end{array}$ & $\begin{array}{l}\text { An important overhead is } \\
\text { distinguished due to the dynamic size } \\
\text { of control packets. }\end{array}$ \\
\hline & Ref. [134] & Multiple UAVs/Mobile & Vehicles & Urban & Performance improvement. & $\begin{array}{l}\text { Maximized the throughput while } \\
\text { considering the delay constraint of } \\
\text { data flows. }\end{array}$ & $\begin{array}{l}\text { A high delay is induced due to the } \\
\text { adopted SCF technique between } \\
\text { vehicles. }\end{array}$ \\
\hline & Ref. [135] & Multiple UAVs/Mobile & $\begin{array}{c}\text { Hybrid } \\
\text { (Vehicles/RSUs) }\end{array}$ & Highway & Avoiding non-cooperative vehicles. & $\begin{array}{l}\text { Improved the connectivity } \\
\text { performance in the presence of } \\
\text { uncooperative vehicles. }\end{array}$ & $\begin{array}{l}\text { Only restricted mobility of UAVs is } \\
\text { considered. }\end{array}$ \\
\hline & Ref. [136] & Multiple UAVs/Mobile & $\begin{array}{c}\text { Hybrid } \\
\text { (Vehicles/RSUs) }\end{array}$ & Highway & $\begin{array}{l}\text { Enhancing the connectivity of ground } \\
\text { vehicular networks }\end{array}$ & $\begin{array}{l}\text { Bridged the communication gaps } \\
\text { between ground vehicles by using } \\
\text { UAVs. }\end{array}$ & $\begin{array}{l}\text { Increased delivery delay when UAVs } \\
\text { have low speeds. }\end{array}$ \\
\hline & Ref. [137] & Multiple UAVs/Mobile & Vehicles & Urban & $\begin{array}{l}\text { Finding reliable routing path in the } \\
\text { sky between UAVs }\end{array}$ & $\begin{array}{l}\text { Avoided obstacles on the ground and } \\
\text { ensured a high delivery ratio. }\end{array}$ & $\begin{array}{l}\text { Introduced an important overhead due } \\
\text { to the adopted discovery process. }\end{array}$ \\
\hline \multirow{5}{*}{ Ground networks } & Ref. [142] & Single UAV/Mobile & Sensor nodes & Hostile area & $\begin{array}{l}\text { Reducing both energy consumption } \\
\text { and radio frequency interference }\end{array}$ & $\begin{array}{l}\text { The UAV collected data effectively in } \\
\text { WSNs. }\end{array}$ & $\begin{array}{l}\text { Considered only static data traffic } \\
\text { loads. }\end{array}$ \\
\hline & Ref. [143] & Multiple UAVs/Mobile & Multiple sensors & Harsh area & $\begin{array}{l}\text { Providing a reliable connection } \\
\text { between ground sensors and the BS }\end{array}$ & $\begin{array}{l}\text { Maintained a connectivity of UAVs as } \\
\text { well as ensuring the relay of data } \\
\text { between sensors and the BS. }\end{array}$ & $\begin{array}{l}\text { The UAV network is considered to be } \\
\text { connected all time, which is not } \\
\text { reasonable. }\end{array}$ \\
\hline & Ref. [144] & Multiple UAVs/Mobile & $\begin{array}{l}\text { Mobile/Static sensor } \\
\text { nodes }\end{array}$ & Disaster area & $\begin{array}{l}\text { Providing facilities for quick response } \\
\text { and event detection }\end{array}$ & $\begin{array}{l}\text { Addressed the success of data delivery } \\
\text { ratio and the reliability of aggregated } \\
\text { data. }\end{array}$ & $\begin{array}{l}\text { Cannot provide a routing hole repair } \\
\text { occurred in UAV networks. }\end{array}$ \\
\hline & Ref. [145] & Two UAVs/Mobile & Ground nodes & Two isolated islands & $\begin{array}{l}\text { Establishing a connection between two } \\
\text { isolated groups of ground nodes }\end{array}$ & $\begin{array}{l}\text { Increased the data transmission and } \\
\text { recovered links between isolated } \\
\text { clusters. }\end{array}$ & $\begin{array}{l}\text { Did not support delay-sensitive } \\
\text { applications. }\end{array}$ \\
\hline & Ref. [147] & Single UAV/Static & Ground nodes & Battlefield area & $\begin{array}{l}\text { Avoiding bandwidth restriction by } \\
\text { using a two-level heterogeneous } \\
\text { wireless network. }\end{array}$ & $\begin{array}{l}\text { Improved the scalability and } \\
\text { throughput by minimizing the number } \\
\text { of transmissions and by adopting UAV } \\
\text { broadcasting, respectively. }\end{array}$ & $\begin{array}{l}\text { Complex architecture requiring high } \\
\text { processing and a lot of exchanging } \\
\text { messages. }\end{array}$ \\
\hline
\end{tabular}

Findings: It is worth noting that the lessons learned from this subsection are listed as follows:

- Optimizing the routing process over any kinds of terrestrial networks is a key design consideration as it significantly affects the performance of UAV-assisted networks. The UAV's placement is an important factor to improve coverage and network performances.

- While optimizing the UAVs' placements, other challenges, such as the appropriate air-to-ground channel, the defined trajectories, GUs locations, and energy consumption of UAVs.

- The frequent fragmentation of the terrestrial network should be also considered.

\section{Open Research Challenges}

The specific mobility models and the different areas of movements of UAVs and terrestrial nodes constitute the major challenges during the design of a routing protocol. Also, the different channels of communications and the existing obstructions on the ground can slow down the routing process deployed in this heterogeneous architecture. Among the problems that hinder the transition of data packets between communicating nodes are the introduced additional delays, the extra overhead, the energy constraints of UAVs and ground nodes, and a lot of exchange of messages in the case when a global knowledge of the topology is required. Consequently, there is a serious need to design efficient routing protocols considering all the aforementioned issues to minimize both the packet losses and the delivery delays.

\section{UAV DATA GATHERING}

Equipped with smart sensors, on-board wireless transceivers, and antennas, UAVs are able to sense, collect, and process data from WSNs or from IoT devices, which are located at a terrestrial location [148]. Moreover, UAVs have the ability to be quickly deployed, moved, and relayed the collected data to a remote central server [149]. However, many issues need to be addressed at different sides as follows:

- Increasing the amount of data to be gathered.

- Jointly optimizing the trajectory and energy consumption of UAVs acting as data collectors.

- Efficiently organizing ground sensors in order to reduce their energy consumption.

- Considering the various obstructions surrounding the ground sensors and ensuring LoS communications during data gathering.

Several contributions which have been proposed in the literature are briefly summarized in Table VI.

\section{A. UAV-WSN Coordination}

For a better energy saving of ground sensors, it is preferable to use UAVs as data collectors having the ability to move closer to sensors, to collect data from them, and thus saving the transmission energy of all sensors (c.f., Fig. 8) [150]. Consequently, optimizing the UAV's trajectory [151], adopting a short-distance LoS [152], and adopting the appropriate UAVto-ground channel model [153], have to be all considered in designing such kind of cooperation. Various contributions using the concept of UAV-WSN coordination have been proposed in the literature and they are discussed in this subsection.

To guarantee that a maximum of data is collected from each ground sensor by UAVs, the authors of [154] jointly optimized the wake-up and sleep schedule of ground sensors and the trajectory of UAVs in order to both efficiently minimize the energy consumption of all nodes and ensure that the maximum amount of data are gathered from each ground sensor. It was shown that the authors assume that UAVs are flying at the same altitude, which is not realistic. Similarly, in [155], an energy-efficient data gathering protocol using UAVs is proposed. UAVs are deployed over WSNs with the aim of reducing the energy consumption of ground sensors that are organized into clusters. It is worthy to mention that this technique has been widely adopted in the context of WSNs 


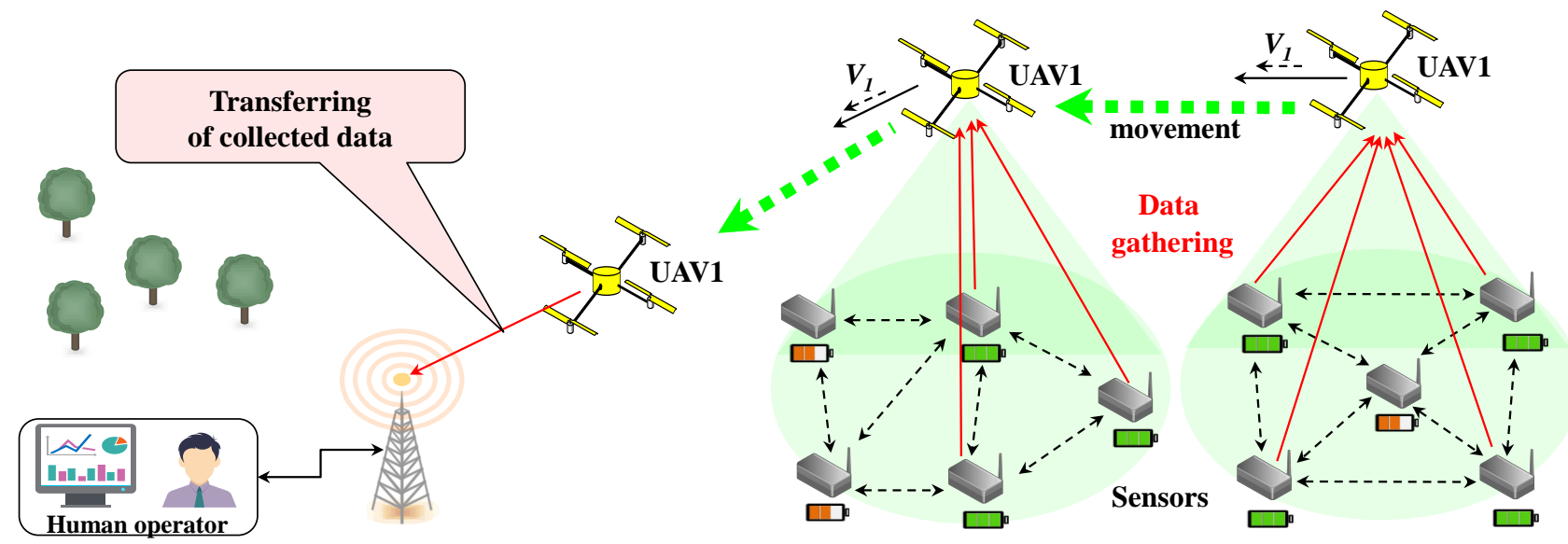

Fig. 8: UAV-WSN coordination.

[156], [157]. Indeed, this strategy derives the optimal path for UAVs to collect data only from cluster-heads. However, the trajectory is not defined according to the energy levels of ground sensors. Another problem is distinguished during the data collection from WSNs. Indeed, to ensure energy efficiency, ground sensors are organized into clusters, which can generate a high overhead during the formation of such groups. For instance, the work in [158] presented a framework for using a UAV as a data collector in WSN. The UAV uses multi-objective decision to generate the UAV path in order to balance the energy consumption among sensor nodes and the UAV. To achieve this goal, a subset of sensor nodes needs to be defined which will play the role of cluster heads within the WSN in which the UAV can use as a waypoint for data collection. However, the cost to form clusters is not negligible. In the case of emergency events, the authors of [159] proposed a UAV-assisted data collection for WSN cluster. This work along with its cloud backend support, considers both the terrestrial network deployment and the optimal flying parameters of the deployed UAV. However, the use of a single UAV can negatively impact the efficiency of data collection, and especially in a large area. As a further improvement, Say et al. [160] proposed a data gathering framework in WSNs in which UAVs are used to collect data from ground sensors. This work allows for removing redundant data transmissions between the sensors in order to both reduce the energy consumption of UAVs and avoid the contention problem caused by simultaneous data transmission. Moreover, a priority based transmission is adopted for sensors to reduce the packet losses, to increase the data throughput, and to improve the energy consumption of sensors. However, during the data gathering, the energy levels of ground sensors are neglected, which can distort the obtained results.

Findings: Three main lessons are learned from this subsection, which are summarized as follows:

- In order to optimize the UAV-WSN data gathering, several parameters and constraints, such as the density of UAVs, the energy restrictions, and the induced overhead have to be considered. To collect a maximum amount of data, the UAV trajectory needs to be optimized according to both the energy levels of ground sensors and the UAV itself.

- The wake-up and sleep schedule of ground sensors needs to be synchronized according to the positions of the UAV

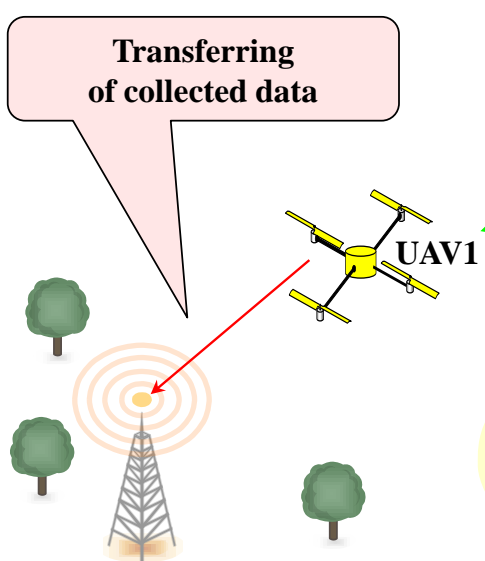

Base station

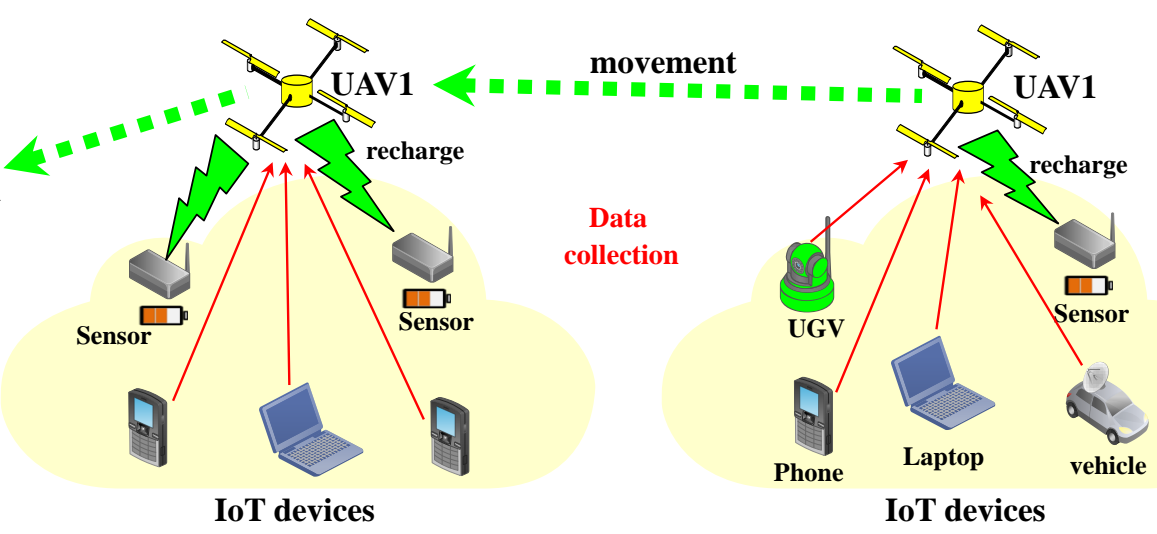

Fig. 9: UAV-IoT coordination. 
collector and not with exchanging messages in order to reduce the overhead.

- An important number of contributions organize the ground sensors into clusters, but the cost in terms of the overhead of this organization is important. Consequently, in order to reduce the degree of induced overhead, reliable clustering solutions need to be proposed.

\section{B. UAV-IoT Coordination}

Due to the restricted capacity of IoT devices in terms of energy and transmission range, classical multi-hop forwarding techniques can cause both unreliable wireless links and waste of their energy resources, leading to low data gathering rates and reduced network lifetime, respectively [161]. As a result, it is crucial to effectively address three important challenges: (i) the definition of appropriate locations such that IoT devices can effectively save energy, (ii) Finding a way such that IoT devices' batteries can be recharged wirelessly and remotely, and (iii) the proposition of reliable data-gathering techniques to collect data from IoT devices [162]. To address these issues, UAVs are considered as the most suitable candidates to dynamically hover around them, recharge them wirelessly using harvesting modules [163], collect data, and transfer them over a long distance to other IoT devices or BSs outside the transmission ranges of these IoT devices (see Fig. 9). In the following, the major UAV-IoT coordination contributions are discussed.

A tradeoff between data collection and wireless energy transfer in IoT environments is proposed by Arabi et al. [164]. Indeed, this framework is composed of UAV-BSs with energy harvesting module which serve the ground IoT devices. Due to their restricted energy, the IoT devices can rely on UAVBSs as energy sources in the case when their battery level under a given threshold. Moreover, UAV-BSs collect data from IoT devices that have residual energy levels above the defined threshold to transmit their packets. However, it was illustrated that the energy consumption of UAV-BSs is neglected. As an improvement of this work, depleted IoT devices can be recharged while ensuring an efficient data gathering by UAVs. Indeed, the same authors in [165] proposed to exploit a UAVBS to perform both data gathering and recharging depleted IoT ground devices. Indeed, the UAV-BS is equipped with an energy harvesting module having the capability of wireless recharging. Moreover, the UAV-BS both exploits the radio frequency emitted by IoT devices to extract energy and collects data from only those having sufficient residual energy to transmit their packets. The UAV path is optimized in order to perform its travel with a minimum of time while improving the network lifetime. In this work, it was distinguished that IoT devices are always on a wake-up mode, thus consuming a lot of energy. Another problem is distinguished in this kind of coordination is the congestion during the channel access, which is addressed in [166]. Indeed, the authors proposed an algorithm which adjusts the speed of UAVs and collect data efficiently. The communication between UAVs and ground sensors is supported by the existing cellular networks. Moreover, UAVs have the ability to efficiently update the speed of ground sensors according to their density during the data collection and coverage. However, the channel status in speed control is not considered by this scheme. To overcome this problem, the work in [167] used UAVs to fly over the sensed area for data collection. Due to the time flight restriction and movement constraint, UAVs require the smoothest and shortest paths to perform their given missions. Thus, Traveling Salesman Problem (TSP) is used to plan the shortest path, while for smoothing the path, Bezier curves are used to convert the paths that are flyable. As a shortcoming, the residual energy of ground sensors and the data gathering time are not considered.

Findings: Important lessons learned can be extracted from this subsection as follows:

- UAV-IoT data gathering requires addressing various problems pertaining to the energy consumption of IoT devices and UAVs, wireless battery charging management, and appropriate trajectories of UAVs for data collection.

- Defining both a near-optimal density of UAVs to perform data collection and a reliable strategy for data exchange

TABLE VI: Summary of contributions to UAV data gathering.

\begin{tabular}{|c|c|c|c|c|c|c|c|}
\hline & & $\begin{array}{l}\text { Density and Mobility of } \\
\text { UAVs }\end{array}$ & $\begin{array}{l}\text { Types of ground } \\
\text { nodes }\end{array}$ & $\begin{array}{l}\text { Deployment } \\
\text { environment }\end{array}$ & Objective & Advantage & Drawback \\
\hline \multirow{5}{*}{ UAV-WSN } & Ref. [154] & Single UAV/Mobile & Multiple sensors & Large area & $\begin{array}{l}\text { Optimizing the UAV's trajectory and } \\
\text { the wake-up schedule to reduce the } \\
\text { energy consumption }\end{array}$ & $\begin{array}{l}\text { Efficiently saved significant energy of } \\
\text { both sensors and UAV, while } \\
\text { guaranteeing that a maximum of data } \\
\text { is collected from each sensor. }\end{array}$ & $\begin{array}{l}\text { UAVs are supposed to be at the same } \\
\text { altitudes. }\end{array}$ \\
\hline & Ref. [155] & Single UAV/Mobile & Multiple sensors & Dangerous area & $\begin{array}{l}\text { Using a UAV as a data mule } \\
\text { collecting data in a clustered WSN } \\
\text { with the aim to save the consumed } \\
\text { energy by sensors. }\end{array}$ & $\begin{array}{l}\text { Minimized the data gathering delay } \\
\text { and reduced energy consumption in } \\
\text { sensors. }\end{array}$ & $\begin{array}{l}\text { The trajectory of the UAV is not } \\
\text { optimized according to the energy } \\
\text { levels of sensors. }\end{array}$ \\
\hline & Ref. [158] & Single UAV/Mobile & Multiple sensors & Large area & $\begin{array}{l}\text { Generating the UAV path such that to } \\
\text { collect data from sensors acting as } \\
\text { cluster-heads. }\end{array}$ & $\begin{array}{l}\text { Decreased the consumed energy } \\
\text { among sensors by selecting only a } \\
\text { subset of sensors to send data. }\end{array}$ & $\begin{array}{l}\text { Generated high overhead during the } \\
\text { election of the cluster-heads. }\end{array}$ \\
\hline & Ref. [159] & Single UAV/Mobile & Multiple sensors & Disaster area & $\begin{array}{l}\text { Using cloud infrastructures to save and } \\
\text { process a large amount of collected } \\
\text { data }\end{array}$ & $\begin{array}{l}\text { Reduced the flying time, the duration } \\
\text { of data collection, and the energy } \\
\text { consumption of sensors and UAVs. }\end{array}$ & $\begin{array}{l}\text { Restricted to a single UAV in a large } \\
\text { area. }\end{array}$ \\
\hline & Ref. [160] & Single UAV/Mobile & Multiple sensors & Sensor area & $\begin{array}{l}\text { Removing redundant sensors and } \\
\text { minimizing transmission distance to } \\
\text { reduce the energy consumption. }\end{array}$ & $\begin{array}{l}\text { Increased both the network } \\
\text { performance and optimized energy } \\
\text { consumption. }\end{array}$ & $\begin{array}{l}\text { Did not consider the energy levels of } \\
\text { sensors during the data transmission. }\end{array}$ \\
\hline \multirow[b]{4}{*}{ UAV-IoT } & Ref. [164] & Single UAV/Mobile & IoT devices & Island & $\begin{array}{l}\text { Collecting data and charging depleted } \\
\text { IoT devices. }\end{array}$ & $\begin{array}{l}\text { Brought more gain in terms of } \\
\text { collection-recharging tradeoff when } \\
\text { using the principle of low battery first. }\end{array}$ & $\begin{array}{l}\text { Did not consider the case when the } \\
\text { UAV has a low residual energy level. }\end{array}$ \\
\hline & Ref. [166] & Single UAV/Mobile & IoT sensors & Harsh environment & $\begin{array}{l}\text { Increasing the data gathering } \\
\text { efficiency while reducing the } \\
\text { congestion during the channel access }\end{array}$ & $\begin{array}{l}\text { Provided accurate and efficient data } \\
\text { collection and avoided any congestion } \\
\text { problem. }\end{array}$ & $\begin{array}{l}\text { Did not consider the channel status in } \\
\text { speed control. }\end{array}$ \\
\hline & Ref. [167] & Multiple UAVs/Mobile & Multiple sensors & City area & $\begin{array}{l}\text { Achieving a faster data collection } \\
\text { while improving the delivery ratio and } \\
\text { achieving a low energy usage }\end{array}$ & $\begin{array}{l}\text { Decreased considerably the consumed } \\
\text { energy and effectively controlled } \\
\text { UAVs to their final destinations. }\end{array}$ & $\begin{array}{l}\text { The energy of ground sensors and the } \\
\text { data collection time are not } \\
\text { considered. }\end{array}$ \\
\hline & Ref. [165] & Single UAV/Mobile & IoT devices & Harsh environment & $\begin{array}{l}\text { Recharging depleted IoT devices and } \\
\text { ensuring an efficient data collection }\end{array}$ & $\begin{array}{l}\text { Provided good performance in terms } \\
\text { of data collection and recharging } \\
\text { energy under different scheduling } \\
\text { policies. }\end{array}$ & $\begin{array}{l}\text { IoT devices are always on a wake-up } \\
\text { mode and did not sleep during the } \\
\text { data collection, thus consuming a lot } \\
\text { of energy. }\end{array}$ \\
\hline
\end{tabular}


between UAVs to relay data with other IoT devices located far away.

- Multiple IoT devices can generate interference issues with UAVs and other IoT devices, thus channel access management needs to be carefully investigated.

\section{Open Research Challenges}

Due to their flexible mobility, UAVs are often deployed over a given region in order to be optimally monitored. Due to the complexity of surrounding obstructions and bad weathers that permanently prevent the LoS between UAVs and the monitored targets, ground sensors are deployed to collect information about the area of interest and transmit them to the closest UAV. However, several issues are distinguished in this kind of UAV assistance paradigm, such as the definition of the optimal altitude of UAVs, the optimal number of UAVs to be deployed, the optimal trajectory of UAVs according to the energy levels of UAVs and ground sensors, and the appropriate channels to communicate and avoid interference problems. Consequently, it is very important to perform deep analysis to enumerate other additional issues and to propose suitable data gathering approaches.

\section{UAV MONITORING}

To facilitate the tasks of emergency services and homeland security organizations on the ground, a set of UAVs equipped with cameras and different other sensors are deployed in the sky, monitoring a given geographical area, and collaborating with different ground entities in a real-time [168]. To guarantee that intercepted images by UAVs respect a certain threshold of visibility and quality, several metrics should be considered, such as the efficiency of the camera, the visibility angle, and the altitude of UAVs [169]. However, to completely cover a given area, a number of important objectives should be clearly formulated as follows:

- Accurately defining the density of UAVs to be deployed while considering their energy consumption and other maintenance costs [170].

- Avoiding the excessive consumption of energy by UAVs.

- Enhancing the movements of UAVs in order to increase their monitoring coverage.

- Designing efficient computing resources in order to gather, process, and store a huge amount of data.

To this end, a significant number of techniques have been proposed, which are summarized in Table VII.

\section{A. Traffic Monitoring}

To monitor a high volume of road traffic and the accidents that it may subsequently provoke, UAVs are considered as a viable and less-time consuming solution to provide an eye-in-the-sky to the problem [171] (see Fig. 10). Indeed, several proposed contributions, taking into account the energy constraints, the communication between UAVs, the traffic load to monitor, and the adequate trajectory to take, are discussed in the following subsections.
To perform the monitoring task while ensuring the energy efficiency, the scheme proposed in [172] aims to use a set of UAVs as LoRaWAN gateways to enhance the energy efficiency of an Intelligent Transportation Systems (ITS) monitoring network. Indeed, UAVs are deployed over specific areas where the traffic load is high based on the area-stress modeling technique for the sole purpose of conserving energy. As for shortcomings, this work neglected the backhaul formed with UAVs and LoRaWAN switches. Similarly, the authors of [173] proposed a technique of road traffic monitoring using UAVs in order to address the issues of managing vast road networks. Indeed, this technique analyzes both real-time traffic of vehicles on the ground based on terrestrial sensor networks and graph theory to model the road network. Also, different security issues are analyzed, e.g., a vehicle without RFID entering a specified area is considered as a potential security risk. It is worthy to note that vehicles are not considered as communicating entities, which can enhance the monitoring purpose. To perform the monitoring mission accurately, a group of UAVs can be allocated. For instance, in [174], the authors considered the use of UAVs for traffic monitoring. This technique uses a combination of inventory routing and the capacitated arc routing problem for road traffic monitoring. For instance, when an edge (i.e., a road segment) is monitored multiple times, it has a negative impact on the performance of the system. However, the density of UAVs needs to be critically analyzed according to the scale of traffic that should be monitored. To avoid the problem of excessive energy consumption and the permanent deployment of UAVs, the work in [175] proposed dynamic UAV-based monitoring by both formulating the first deterministic arc routing problem and obtaining its stochastic dynamic policy. Moreover, this work uses a programming algorithm based on Monte Carlo squares simulations so that UAVs can improve monitoring satisfaction.

Findings: Various learned lessons from this subsection are summarized as follows:

- The use of UAVs to perform traffic monitoring introduces new challenges. For instance, the connectivity between UAVs needs more investigation in order to reliably transmit traffic information to the human operator [176].

- Since existing vehicles are not considered communicating entities in the majority of contributions, there is a need for designing UAV-vehicle cooperation to provide more accurate information about traffic.

- A mathematical modeling should be elaborated to extract an accurate relationship between the density of traffic and the density of UAVs that are required to be deployed.

\section{B. Environment Inspection}

As shown in Fig. 11, the monitoring of complex environments is an important application in which the basic functionality is to recognize and track multiple static or moving targets [177]. This kind of applications has some challenging properties, such as the restricted flight zones [178], the event rate [179], and the risk areas [180] (e.g., high populated area). However, there is a serious need to equip UAVs with 


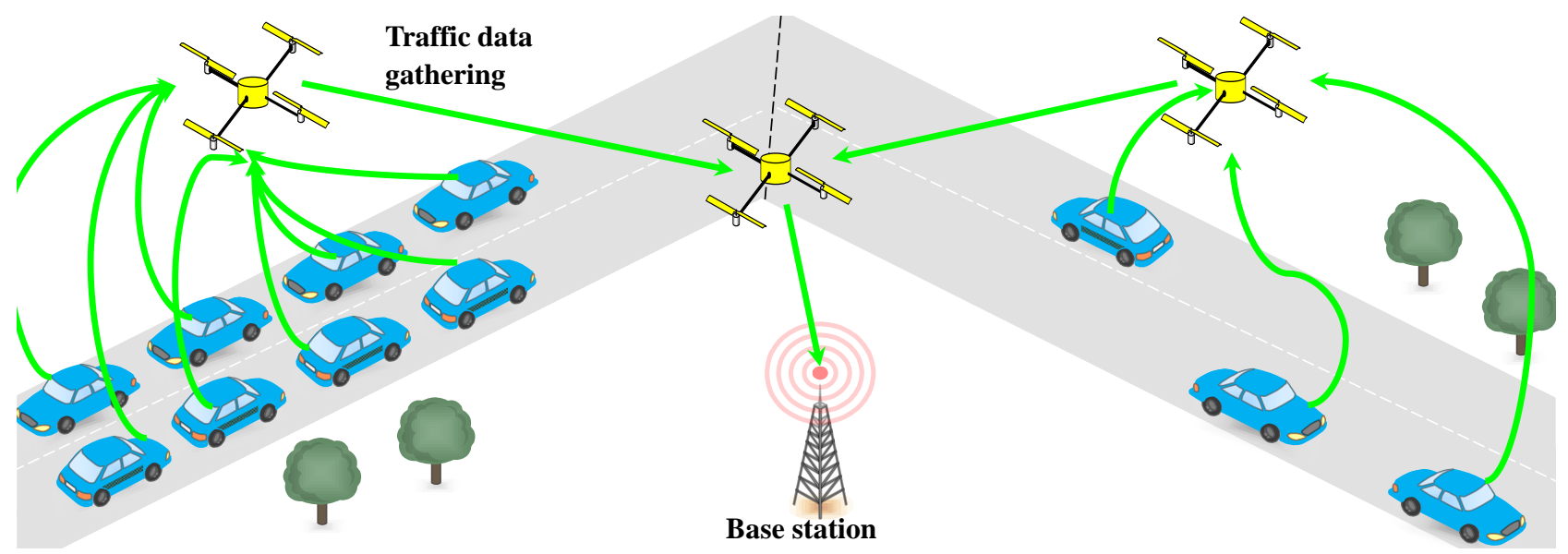

Fig. 10: UAV-assisted traffic monitoring.

the required devices and various types of loads to efficiently accomplish the task. In the following, the major contributions in this field are described and discussed.

Another use case where UAVs can be deployed for inspection and monitoring is the indoor/outdoor environments. For instance, Yue et al. [181] proposed a distributed system to detect trespassing UAVs and their approximate positions based software defined radio (SDR) transceivers and wireless acoustic sensors. A machine learning algorithm is integrated with the SDR transceivers to decrypt the telemetry protocols of the UAV in question. Based on the decrypted information, UAVs can send control commands in order to eliminate the intruder UAV. However, in the case when intruder UAVs are using an unknown protocol, they cannot be detected. Another interesting monitoring framework using UAV-based networks is proposed in [182]. This framework is deployed for smart city monitoring using public transportation and city infrastructures in order to support the battery charge and communications of UAVs. Nevertheless, it was shown that the energy restriction capacity of UAVs is not considered. In order to track multiple moving targets using UAVs, the work in [183] used a cooperative network system of multi-UAV monitoring. Indeed, this system is based on both the animal colony perception and moving small target recognition techniques using the fusion of multiple data sources. Moreover, due to the complexity of the monitored area, this work adopts appropriate algorithms based on machine learning. However, the problem of energy consumption always persists. Furthermore, the main problem with ISR missions is how to find routes that allow UAVs to collect data packets of a set of task locations and transmit them directly to the control station (BS). In [184], a centralized path planning problem is formulated for gathering data while minimizing the delivery delay and satisfying the cycle length constraints of each task. Nevertheless, the authors have shown that the lower bounds for computational efficiency need to be strengthened.

Findings: A notable number of lessons learned from this subsection are summarized as follows:

- Given the restricted on-board energy of UAVs, the energy efficiency requires careful consideration. Indeed, the less energy consumption of UAVs, the more flight time is, and the more monitored areas will be.

- Two different monitoring techniques can be used for environmental inspection: (i) Interception of messages and (ii) camera-based surveillance. Both techniques have their own challenges that require careful studies, such

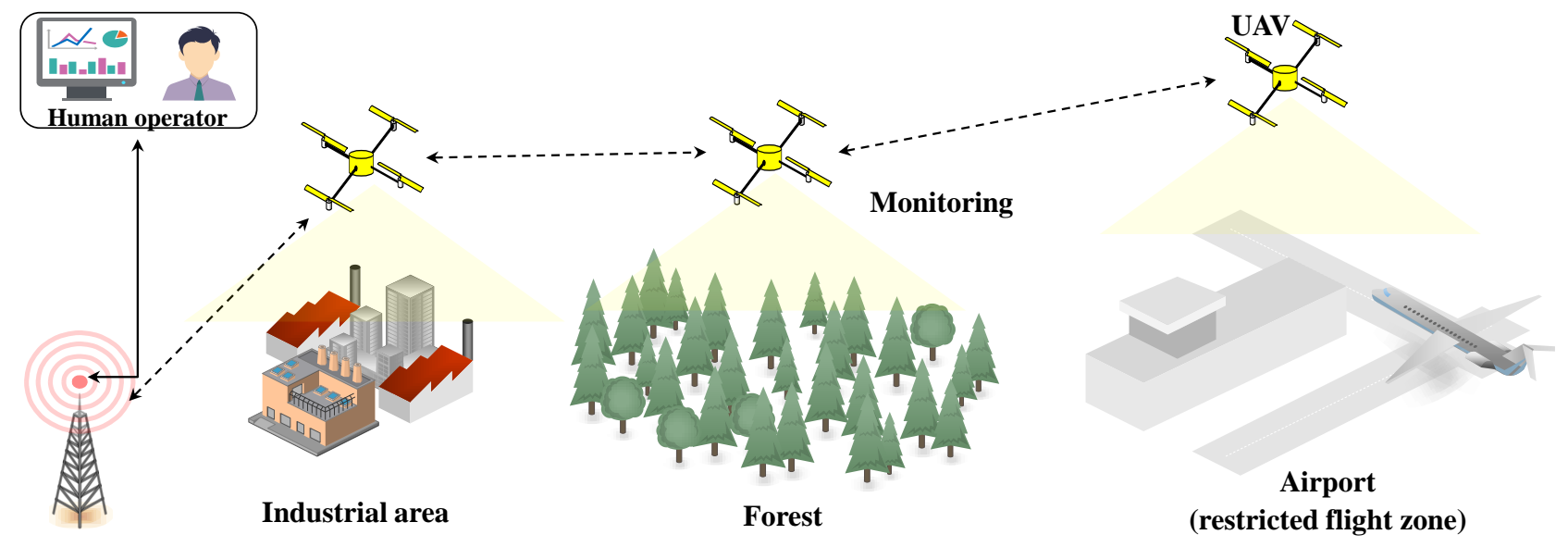

Fig. 11: UAV-assisted environment inspection. 
TABLE VII: Summary of contributions to UAV monitoring.

\begin{tabular}{|c|c|c|c|c|c|c|c|}
\hline & & $\begin{array}{l}\text { Density and Mobility of } \\
\text { UAVs }\end{array}$ & $\begin{array}{l}\text { Types of ground } \\
\text { nodes }\end{array}$ & $\begin{array}{c}\text { Deployment } \\
\text { environment }\end{array}$ & Objective & Advantage & Drawback \\
\hline \multirow{4}{*}{ Traffic monitoring } & Ref. [172] & Multiple UAVs/Mobile & $\begin{array}{l}\text { Multiple sensors and } \\
\text { vehicles }\end{array}$ & Urban area & $\begin{array}{l}\text { Monitoring in IoT-ITS with an energy } \\
\text { efficient perspective }\end{array}$ & $\begin{array}{l}\text { Provided energy efficient surveillance } \\
\text { by using UAVs as LoRaWAN } \\
\text { gateways. }\end{array}$ & $\begin{array}{l}\text { Did not consider the backhaul formed } \\
\text { with UAVs and LoRaWAN switches. }\end{array}$ \\
\hline & Ref. [173] & Single UAV/Mobile & Multiple sensors & Urban area & $\begin{array}{l}\text { Analyzing real-time traffic and } \\
\text { security issues. }\end{array}$ & $\begin{array}{l}\text { Provided real-time and quick } \\
\text { responding to several security scenario } \\
\text { situations. }\end{array}$ & $\begin{array}{l}\text { Did not consider vehicles as } \\
\text { communicating entities, which can } \\
\text { improve monitoring. }\end{array}$ \\
\hline & Ref. [174] & Multiple UAVs/Mobile & BS/Vehicles & Urban area & $\begin{array}{l}\text { Allocating a group of UAVs for roads' } \\
\text { monitoring }\end{array}$ & $\begin{array}{l}\text { Provided an effective decision-making } \\
\text { method for solving the UAV } \\
\text { scheduling problem. }\end{array}$ & $\begin{array}{l}\text { Cannot support large scale traffic due } \\
\text { to the limited number of UAVs that } \\
\text { can be deployed. }\end{array}$ \\
\hline & Ref. [175] & Multiple UAVs/Mobile & Vehicles & Urban area & $\begin{array}{l}\text { Systematically deploying UAVs over } \\
\text { multiple periods for road monitoring }\end{array}$ & $\begin{array}{l}\text { Effectively succeeded in real-time } \\
\text { monitoring using the different } \\
\text { proposed techniques. }\end{array}$ & $\begin{array}{l}\text { The energy consumption of UAVs is } \\
\text { not considered during the monitoring. }\end{array}$ \\
\hline \multirow{4}{*}{ Environment inspection } & Ref. [181] & Single UAV/Mobile & SDR transceivers/BSs & Sensitive area & $\begin{array}{l}\text { Detecting trespassing UAVs using } \\
\text { three techniques }\end{array}$ & $\begin{array}{l}\text { Provided an integrated framework for } \\
\text { amateur UAV surveillance. }\end{array}$ & $\begin{array}{l}\text { Cannot detect unwelcome UAVs } \\
\text { transmitting data with an unknown } \\
\text { protocol. }\end{array}$ \\
\hline & Ref. [182] & Multiple UAVs/Mobile & $\begin{array}{l}\text { Vehi- } \\
\text { cles/RSUs/Surface } \\
\text { buoys }\end{array}$ & Urban area & $\begin{array}{l}\text { Supporting continuous monitoring in } \\
\text { smart city and extensive ocean }\end{array}$ & $\begin{array}{l}\text { Provided efficient monitoring } \\
\text { management in both environments. }\end{array}$ & $\begin{array}{l}\text { This work did not consider the energy } \\
\text { restriction of UAVs and the } \\
\text { near-optimal trajectories to save } \\
\text { energy. }\end{array}$ \\
\hline & Ref. [183] & Multiple UAVs/Mobile & Sensors & Complex area & $\begin{array}{l}\text { Recognizing and tracking multiple } \\
\text { moving targets using UAVs and } \\
\text { sensors }\end{array}$ & $\begin{array}{l}\text { Provided a high accuracy monitoring } \\
\text { and good network performance. }\end{array}$ & $\begin{array}{l}\text { The energy of movements and } \\
\text { processing are not considered by this } \\
\text { scheme. }\end{array}$ \\
\hline & Ref. [184] & Multiple UAVs/Mobile & Singe BS & Not specified & $\begin{array}{l}\text { Satisfying the revisit period of each } \\
\text { task. }\end{array}$ & $\begin{array}{l}\text { Well-suitable for a quick on-board } \\
\text { re-planning. }\end{array}$ & $\begin{array}{l}\text { This strategy needs to strengthen the } \\
\text { lower bounds for computational } \\
\text { efficiency. }\end{array}$ \\
\hline
\end{tabular}

as the similarity of adopted frequencies, the transmission range of UAVs, and the congestion problem.

- When the monitoring is based on the mounted camera, a huge amount of data needs to be processed, stored, and transferred to a human operator or BS for analyzing. This requires, e.g., a robust cloud computing concept [185], [186].

\section{Open Research Challenges}

To ensure near-optimal monitoring of a given area, an efficient mechanism of UAV placement needs to be ensured. This mechanism is based on the energy levels of UAVs and the areas that are most likely to be monitored. In addition, the mobility of UAVs, as well as the intermittent connectivity with the ground BS, are required to be guaranteed. If in the case when the surveillance is based on video streaming, UAVs have to transfer a large amount of data flow to the BS, which exposed to different interference problems with existing terrestrial cellular networks. Otherwise, UAVs can exploit their wireless communication devices to detect, e.g., missing victims or explore a given region using sensors. The UAV-assisted monitoring field is still at the infant stage of research and more investigations and results can be done.

\section{UAV CELLULAR COMMUNICATION}

Recently, UAVs have witnessed significant enhancements in terms of payload, energy consumption, and design [187]. As a result, it will be more practical for UAVs to incorporate different kinds of communication equipment for several purposes, such as extending the coverage of existing terrestrial cellular networks, serving congested GUs, and assisting ground BSs in their functioning [188]. To efficiently perform this incorporation, it is a mandatory condition to consider different constraints that are listed as follows:

- Jointly optimizing the placement and trajectory of UAVs according to the requirements of GUs and terrestrial networks.

- Improving access to the shared spectrum with terrestrial cellular networks.

- Minimizing the energy consumption of UAVs.
- Increasing the number of served GUs while enhancing different metrics, such as throughput, latency, and packet losses.

In the following, different contributions related to each constraint are discussed and they are summarized in Table VIII.

\section{A. Placement Optimization}

In order to maximize the capacity and the coverage of existing cellular networks, strategic placement of UAVs needs to be considered [189]. Moreover, in order to provide the required QoS and performance, the minimum number of UAVs to deploy needs to be defined [190]. UAVs can act both as relays of information between the different ground BSs and as connectivity support for cellular users (c.f., Fig. 12). In the following, different major contributions trying to optimize the UAV placement are discussed.

As known to all, the optimal placement of UAVs acting as aerial BSs constitute a key solution to provide a near-optimal coverage of a given area. For instance, the work proposed in [191] studied the strategic placement of a set of UAV-BSs over a large scale ground network composed of ground BSs. To increase the coverage of ground BSs using UAV-BSs, this work is based on a stochastic geometric approach. However, as for GUs, this system only has the ability to cover GUs uniformly distributed. To improve the $5 \mathrm{G}$ network coverage over a given area, the work in [192] designed a novel placement strategy of cooperative UAVs based on demand areas. The strategy is able to provide a notable performance enhancement of network metrics, such as the throughput, the communication delays, and the deployment cost. However, the mobility of GUs is not supported. When it is the case to provide coverage to other UAVs, Mozaffari et al. [193] proposed both a new concept of three-dimensional (3D) cellular networks and 3D cell associations for UAV user equipment (UAV-UE). Moreover, a framework for network planning, mobility management, routing, resource management, and multiple access are considered for UAV-BSs. Nevertheless, the authors omitted the mobility patterns of UAVs and their energy consumption. To enhance the performance of ground cellular networks, a Q learning technique has been applied in [194] to both find the appropriate 3D positions for UAV-BSs and ensure a 


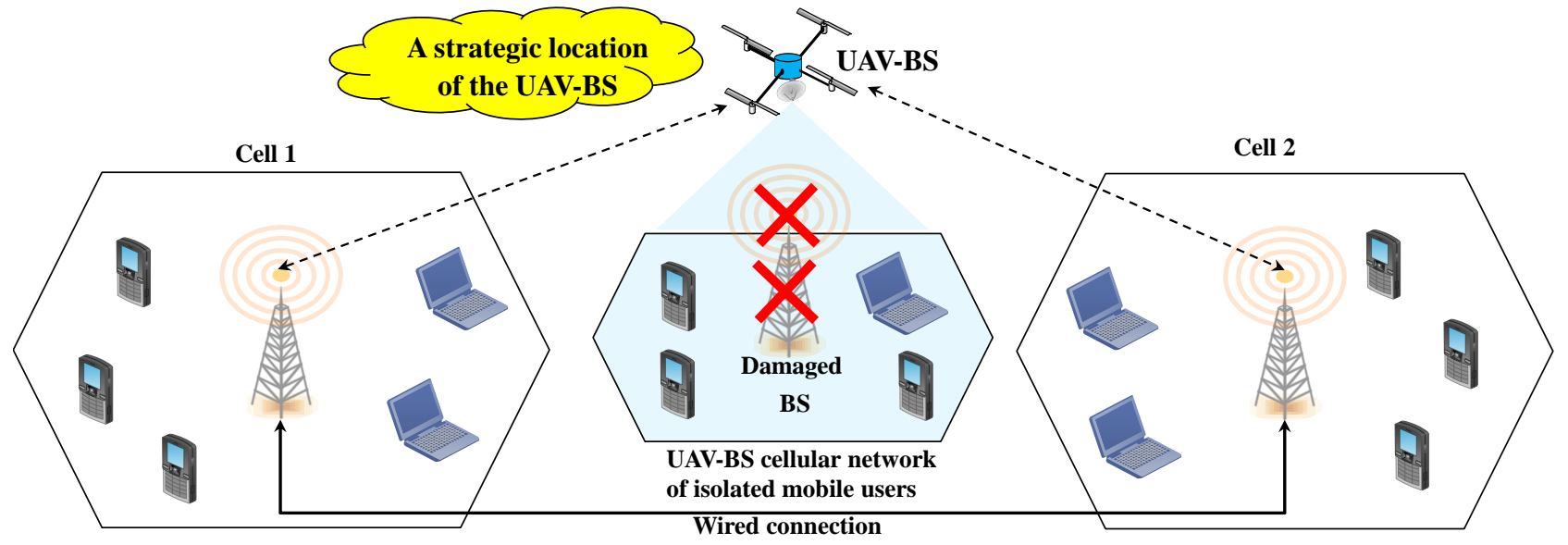

Fig. 12: Placement optimization UAV-assisted cellular networks.

required QoS according to the mobility of GUs. Furthermore, this technique has the ability to both maximize the aggregate throughput of the ground networks and efficiently achieve enhanced short and long term cache hit rate compared with other caching techniques in the literature. Despite its support for the mobility of GUs, this system cannot support a highly mobile GUs, such as vehicles, UAVs, or even running humans. In order to serve and improve the coverage of a particular region and its respective GUs, the work in [195] designed a heuristic framework based on a particle swarm optimization. This technique tries to find the near-optimal number of UAVBSs along with their respective positions and altitudes in order to both avoid interference with other entities and cover a high number of GUs. However, there is no ground BS, which can put this system into question when UAVs are out of service.

Findings: The placement optimization study allowed us to summarize multiple lessons learned during this investigation as follows:

- The near-optimal placement of UAVs needs to be defined according to the non-uniform distribution of GUs and their mobility.

- The trajectories of UAVs, as well as their energy consumption have to be considered during their deployment over a partially covered region by existing cellular networks.

- A robust networking model between UAVs needs to be carefully investigated in order to effectively extend the coverage of a given cellular network.

\section{B. Mobility Optimization}

The trajectory of UAVs has to be thoroughly studied in order to satisfy their mission requirements and at the same time fulfilling the optimum throughput for the ground terrestrial networks and GUs [196]. In addition, the UAV trajectory can be flexibly defined based on the locations of ground BSs as well as the distribution of GUs in order to both ensure optimal and complementary coverage of the associated ground BSs and minimize the interference that may occur between these entities (c.f., Fig. 13). Several contributions are proposed in this field and they are discussed as follows.
By achieving a trade-off between complexity and network performance, the authors of [197] optimized the trajectory of UAVs under a defined communication QoS requirement with ground cellular networks. The idea behind this technique is to minimize the mission flying time by using convex optimization techniques and graph theory. Not surprisingly, the initial and final locations of UAVs and their speeds are not discussed in this work. Similarly, Lyu et al. [198] also studied a mobility optimization employing a hybrid network architecture. This architecture uses UAV-BS to fly over the ground BSs cell edge for data offloading. Also, to increase the common throughput of all cell-edge users, the UAV trajectory, the spectrum allocation, and the user partitioning are jointly optimized. However, this technique cannot deal with different isolated groups of GUs. To increase a ground network throughput and to increase the spectral efficiency of the UAV-BS link, the work in [199] investigated the mobility models of UAV-BSs using an iterative optimization algorithm in which UAVs periodically update their positions. The authors show that larger system throughput is performed by freeing up the movement of UAVBSs. Nevertheless, a user's association scheme needs to be carefully studied. Another promising approach is proposed in [200] to study the optimal UAV-BSs placement and the provided service time by considering the GUs density and their location. Moreover, this deployment can offload a significant amount of data to GUs. It was shown the total in-service time of GUs is significantly increased. However, the trajectories of UAVs are not well investigated, which can be crucial to serving optimally GUs. While Fotouhi et al. [201] proposed a distributed scheme considering the mobility of the UAV$\mathrm{BS}$, the energy efficiency issue, the mobility of GUs, and the interference signals. The main goal of this work is to find the appropriate mobility model for the UAV-BS inside its limited small cell boundaries to enhance the packet throughput for cell-edge users. It was demonstrated that the spectral efficiency is efficiently increased while keeping energy consumption at an acceptable level.

Findings: In summary, three main lessons learned from the mobility optimization of UAVs are listed as follows:

- To efficiently optimize the mobility of UAVs, various 


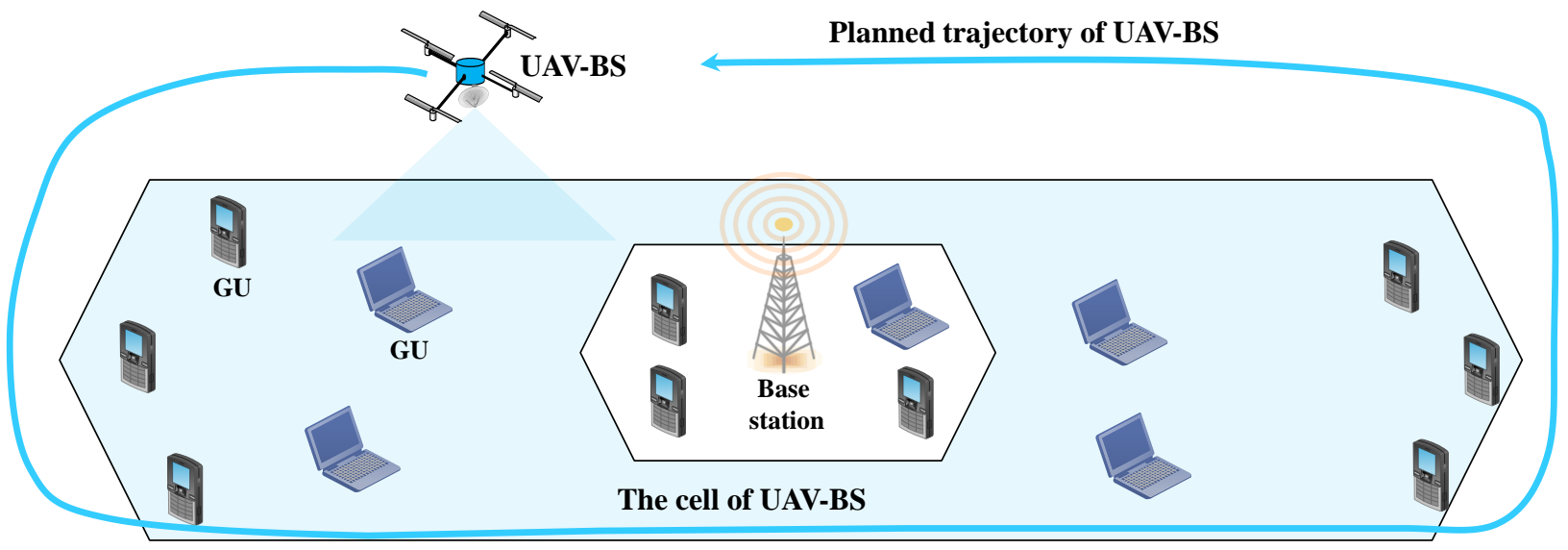

Fig. 13: Mobility optimization UAV-assisted cellular networks.

mathematical models are required to be leveraged, such as Optimization and game theories, machine learning, and stochastic geometry.

- The coordination between multiple UAVs is a mandatory condition to generate near-optimal trajectories to cover the maximum of the surface.

- Different factors need to be considered during the design of a given trajectory, such as the spectral efficiency, the interference issue, transmission power, and obstructions.

\section{Energy Efficiency}

The restricted-energy capacity of UAVs is among the main critical challenges of UAV cellular communications [202]. Indeed, in order to increase its lifetime, the UAV network has to demonstrate its capacity to guarantee an energy-efficient functionality by adopting several techniques ensuring a wellregulated energy consumption between UAVs (c.f., Fig. 14). In this context, multiple proposed energy saving contributions are discussed as follows.

To assist communication between two pair of communicating nodes located on the ground, the authors of [203] jointly optimized the communication time allocation and the trajectory of UAVs in order to maximize both the spectrum and energy efficiency. Moreover, to enhance its backhaul links and end users, this work considered a UAV, single end user, and backhaul in isolation. The authors affirm that the spectrum, the energy efficiency, and the trajectory, are optimized. As a drawback, they display a high delivery delay when the communicating nodes are separated by a large distance. To create an energy-efficient relay network, the proposed work in [204] exploited the cooperation of multiple UAVs to increase the ground network coverage by relaying data packets from ground sensors to a remote BS using time division multiple access (TDMA). Moreover, a cooperative movement of UAVs is adopted to simplify the design of the energy-efficient UAV network. In this work, the computational complexity is reduced, but a crucial challenge is neglected, such as the fragmentation of the UAV network. To both overcome this issue and increase the energy efficiency with equity constraints, a game theoretic data gathering technique is proposed in [205]. This work considered multiple UAVs flying in a circular trajectory and collecting data from a set of ground sensors organized in the form of clusters. The cluster heads are the only responsible to send data to the hovering UAVs based on the allocated time slots. The authors assume that UAVs have static trajectories, which is a drawback against

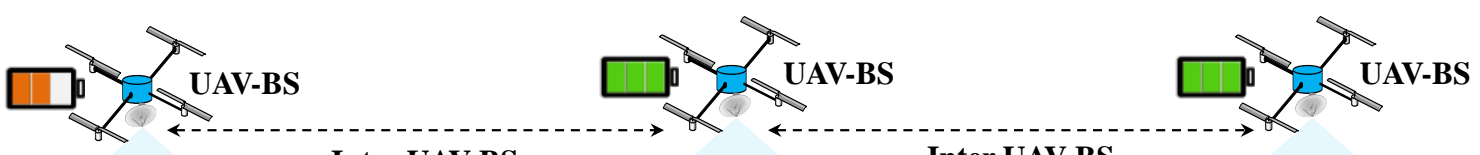

Inter UAV-BS

Inter UAV-BS

communication

communication
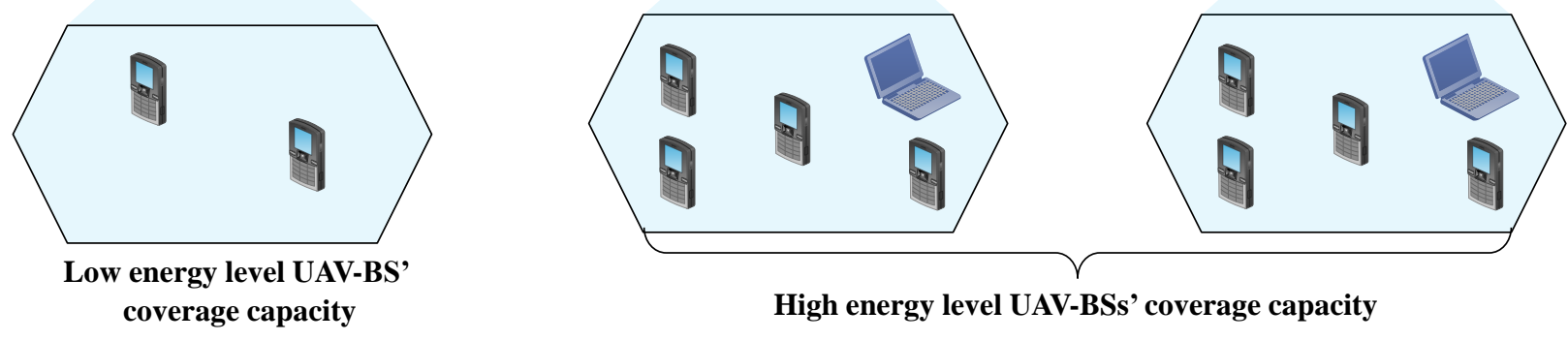

High energy level UAV-BSs' coverage capacity

Fig. 14: Energy efficiency UAV-assisted cellular networks. 
the environmental conditions and the urgent needs of GUs. While the authors in [206] designed a relay selection technique based on the channel conditions for energy-constrained UAVto-ground communication systems. Moreover, this strategy can be efficiently deployed without impacting the required QoS. It was shown that the relay mechanism between UAVs is not exploited.

Findings: An important number of lessons can be learned from the analysis performed above, and especially on how to optimize the energy consumption of UAVs. As a consequence, a brief summary is listed below:

- Many optimized techniques can be adopted for the energy efficient deployment of UAVs, such as the trajectory, the communication time allocation, the processing, and the relay mechanism [207].

- Information related to energy levels has to be shared between UAVs to have a global vision about energy consumption and which UAV has to be replaced.

- The optimal placement of recharge stations can be also investigated [208].

\section{Spectrum Sharing and Access}

As illustrated in Fig. 15, the communications between UAVs and ground BSs are conducted through a dedicated A2G channel that is exposed to different surrounding constraints, such as interfering with other ground BSs [209]. Indeed, the performance of the UAV-assisted cellular network channel can be impacted by the probability of LoS, the altitude of UAVs, and the elevation angle between UAVs and ground BSs [210]. Moreover, it is shown that the radio propagation channel can be distorted by the interference of neighbor cells and shadowing effects [211]. As a consequence, many works are performed with the aim to address spectrum efficiency using different techniques [212]. Also, other works are proposed to study both the different access techniques to the shared spectrum and the common problems encountered.

To study more deeply the performance of aerial radio connectivity using simulations, the authors in [213] selected rural areas with a focus on path loss modeling for both uplink and downlink connections. Several techniques have been considered by the UAV, including the antenna beam selection and interference cancellation based on both the selection of the serving BS's location and the interference suppression at the receiver, respectively. As a result, the overall system performance has been enhanced for both ground and aerial users. Also, it was illustrated that the interference mitigation scheme provided good potential. However, a crucial degradation of the network performance is distinguished when a higher penetration of connected aerial vehicles is required. Another work is proposed in [214] with the aim to analyze the feasibility to provide LTE connectivity for UAVs. Also, this work studied both the wireless connectivity requirements of UAVs and their propagation characteristics using measurements and ray-tracing simulations. Nevertheless, it was shown that the increasing number of UAVs leads to high aerial interference, and thus decreasing the network spectral efficiency. Moreover, HAUs cannot be covered due to the limited LTE coverage. To surpass this problem and to maximize the cellular coverage, the authors in [215] studied the coverage probability of UAVBSs while paying attention to channel modeling combining LoS and non-LoS path loss components. Moreover, this work models downlink inter-cell interference to the A2G aspects. However, nothing is done towards the trajectories of UAV-BSs to optimally increase the coverage of such networks. Another strategy is used in [216], where the authors investigated two multi-UAV relaying approaches of maximizing the data rate in multiple dual-hop links and multi-hop link, in which optimal UAV hovering positions were derived. The authors have omitted the impact of the surrounding environment and the different physical constraints. The work in [217] proposes a multi-layer UAVs hierarchical based on several types of UAVs. The main purpose of this architecture is to integrate UAVs in $5 \mathrm{G}$ and beyond networks by studying the feasibility laser power transfer and radio frequency/microwave-based wireless power transfer. As a drawback, the interference induced by this architecture and the optimization of the 3D deployment of UAVs are omitted.

Findings: The main lessons learned from this subsection are as follows:

- Several techniques can be used during channel access,

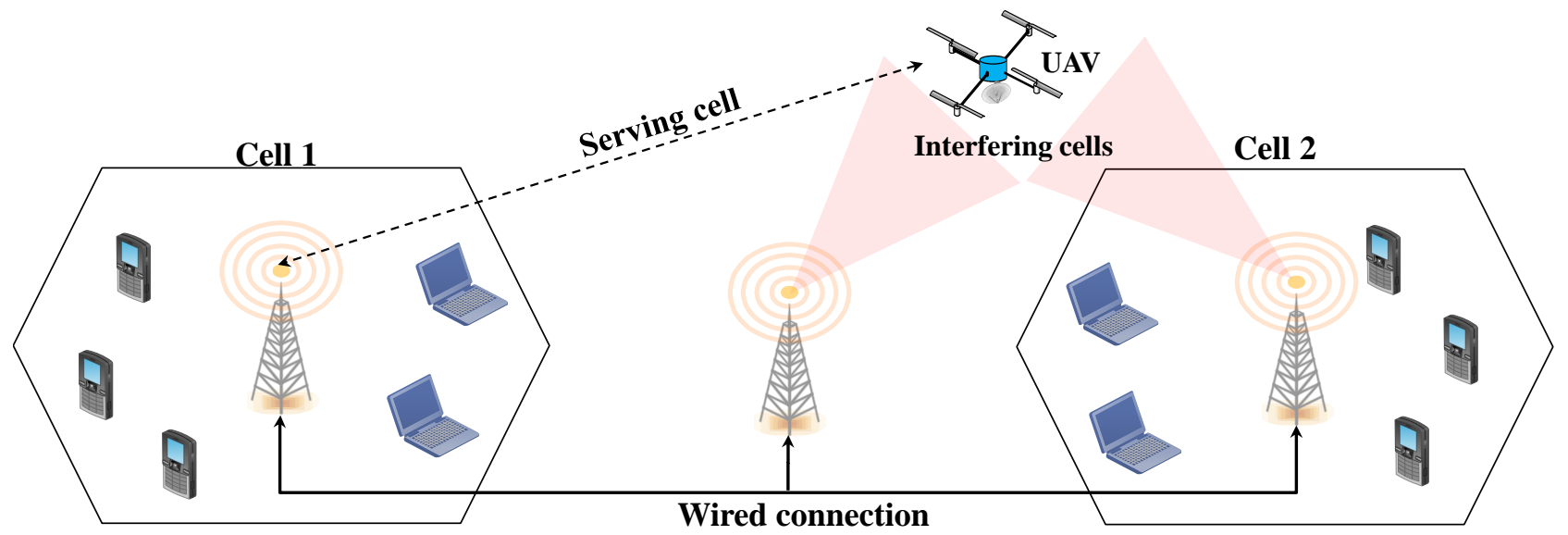

Fig. 15: Spectrum sharing and access in UAV-assisted cellular networks. 
TABLE VIII: Summary of contributions to UAV cellular communication.

\begin{tabular}{|c|c|c|c|c|c|c|c|}
\hline & & $\begin{array}{l}\text { Density and Mobility of } \\
\text { UAVs }\end{array}$ & $\begin{array}{c}\text { Types of ground } \\
\text { nodes }\end{array}$ & $\begin{array}{l}\begin{array}{l}\text { Deployment } \\
\text { environment }\end{array} \\
\end{array}$ & Objective & Advantage & Drawback \\
\hline \multirow[b]{5}{*}{ Placement optimization } & Ref. [191] & Multiple UAV-BSs/Static & BSs & Urban area & $\begin{array}{l}\text { Providing a complementary supply for } \\
\text { terrestrial networks using UAV-BSs }\end{array}$ & $\begin{array}{l}\text { The adopted placement strategy improved the } \\
\text { network performance and its spatial regularity. }\end{array}$ & $\begin{array}{l}\text { This strategy did not consider the non-uniform } \\
\text { distribution of the users. }\end{array}$ \\
\hline & Ref. [192] & Multiple UAV-BSs/Mobile & BSs & Not specified & $\begin{array}{l}\text { Effectively placing UAV-BSS according to the } \\
\text { demand areas }\end{array}$ & $\begin{array}{l}\text { Improved the network throughput and } \\
\text { decreased the network delays. }\end{array}$ & Mobility of GUs is not considered. \\
\hline & Ref. [193] & $\begin{array}{c}\text { Multiple } \\
\text { UAV-BSs/UAV/Mobile } \\
\end{array}$ & None & Large area & $\begin{array}{l}\text { Integrating UAV-BSs and cellular connected } \\
\text { UAVs using 3D cellular networks. }\end{array}$ & $\begin{array}{l}\text { Reduced the latency of cellular connected } \\
\text { UAVs and improved spectral efficiency. }\end{array}$ & $\begin{array}{l}\text { The energy and the mobility limitations of } \\
\text { different kinds of UAVs are not considered. }\end{array}$ \\
\hline & Ref. [194] & Single UAV-BS/Mobile & $\mathrm{BSs} / \mathrm{GUs}$ & Urban area & $\begin{array}{l}\text { Obtaining better performance of ground cellular } \\
\text { networks by optimally placing the UAV-BS }\end{array}$ & $\begin{array}{l}\text { Provided high QoS by considering the } \\
\text { movement of GUs. }\end{array}$ & $\begin{array}{l}\text { The high mobility of GUs is not supported by } \\
\text { the UAV-BS deployed in this strategy. }\end{array}$ \\
\hline & Ref. [195] & Multiple UAV-BSs/Mobile & Multiple GUs & Multiple regions & $\begin{array}{l}\text { Defining the minimum number of UAV-BSs } \\
\text { and their optimal 3D placements }\end{array}$ & Satisfied the QoS requirements of the network. & $\begin{array}{l}\text { No ground BSs is assumed to exist, which is a } \\
\text { disadvantageous factor to satisfy the majority } \\
\text { of GUs. }\end{array}$ \\
\hline \multirow{5}{*}{ Mobility optimization } & Ref. [197] & $\begin{array}{c}\text { Multiple } \\
\text { UAV-BSS/UAV/Mobile }\end{array}$ & Multiple BSs/GUs & Suburban area & $\begin{array}{l}\text { Optimizing the trajectories of UAV-BSs to their } \\
\text { mission completion time. }\end{array}$ & $\begin{array}{l}\text { Jointly optimized complexity and network } \\
\text { performance. }\end{array}$ & $\begin{array}{l}\text { The maximum speed and the initial and final } \\
\text { positions of UAV-BSs are not discussed. }\end{array}$ \\
\hline & Ref. [198] & Single UAV-BS/Mobile & $\begin{array}{l}\text { Single BS/Multiple } \\
\text { GUs } \\
\end{array}$ & Not specified & $\begin{array}{l}\text { Increasing the throughput of GUs by jointly } \\
\text { optimizing the UAV's trajectory }\end{array}$ & $\begin{array}{l}\text { Optimized throughput of GUs only with a } \\
\text { single UAV-BS compared to conventional } \\
\text { cellular networks. }\end{array}$ & $\begin{array}{l}\text { This strategy did not perform well when } \\
\text { different groups of GUS are located in distant } \\
\text { isolated areas, respectively. }\end{array}$ \\
\hline & Ref. [199] & Multiple UAV-BSs/Mobile & Multiple GUs & Urban area & $\begin{array}{l}\text { Studying the mobility model of the UAV-BSs } \\
\text { in order to serve GUS }\end{array}$ & $\begin{array}{l}\text { Performed a larger system throughput by } \\
\text { freeing up the movement of UAV-BSs. }\end{array}$ & $\begin{array}{l}\text { A complex user association scheme should be } \\
\text { carefuly investigated, which requires a global } \\
\text { network knowledge. }\end{array}$ \\
\hline & Ref. [200] & Multiple UAV-BSs/Mobile & Multiple GUs & Not specified & Studying the deployment problem of UAV-BSs & Increased the total in-service time of all GUs. & $\begin{array}{l}\text { The trajectories of UAV-BSs are neglected, } \\
\text { which can be crucial to serving optimally GUs. }\end{array}$ \\
\hline & Ref. [201] & Single UAV-BS/Mobile & Single GU & Urban area & Serving areas of urgent demand using UAV-BS & $\begin{array}{l}\text { Tncreased the spectral efficiency while keeping } \\
\text { the energy consumption at the same level. }\end{array}$ & $\begin{array}{l}\text { The different existing obstructions on the } \\
\text { ground are omitted. }\end{array}$ \\
\hline \multirow[b]{4}{*}{ Energy efficiency } & Ref. [203] & Single UAV/Mobile & Multiple GUs & $\begin{array}{l}\text { Less-infrastructure } \\
\text { area }\end{array}$ & $\begin{array}{l}\text { Assisting the communication between a ground } \\
\text { pair of source and destination }\end{array}$ & $\begin{array}{l}\text { Increased the spectrum and energy efficiency } \\
\text { and optimized the UAV trajectory. }\end{array}$ & $\begin{array}{l}\text { Increased the delay of delivery in the case } \\
\text { when the distance between the communicating } \\
\text { nodes are very large. }\end{array}$ \\
\hline & Ref. [204] & Multiple UAVs/Mobile & Single BS/Sensors & Rural area & $\begin{array}{l}\text { Extending the network lifetime while ensuring } \\
\text { a high delivery ratio. }\end{array}$ & $\begin{array}{l}\text { Reduced the computational complexity with } \\
\text { negligible degradation of the network. }\end{array}$ & $\begin{array}{l}\text { Did not consider a severe fragmentation of the } \\
\text { UAV network. }\end{array}$ \\
\hline & Ref. [205] & Multiple UAVs/Mobile & Sensors & Hostile environment & $\begin{array}{l}\text { Maximizing the energy efficiency in ground } \\
\text { networks. }\end{array}$ & $\begin{array}{l}\text { Provided a near optimal performance in terms } \\
\text { of energy efficiency and throughput. }\end{array}$ & $\begin{array}{l}\text { Static trajectories of UAVs, which cannot } \\
\text { support urgent needs in the ground networks. }\end{array}$ \\
\hline & Ref. [206] & Multiple UAV-BSs/Mobile & Multiple GUs/BSs & Urban area & $\begin{array}{l}\text { Extending the lifetime of the battery operated } \\
\text { aerial-terrestrial communication links }\end{array}$ & $\begin{array}{l}\text { Showed efficient and reliable performance } \\
\text { according to the energy consumption. }\end{array}$ & $\begin{array}{l}\text { Did not exploit the relay mechanism between } \\
\text { UAV-BSs. }\end{array}$ \\
\hline \multirow{5}{*}{ Spectrum sharing and access } & Ref. [213] & Multiple UAV-BSs/Mobile & Multiple GUs/BSs & Rural area & $\begin{array}{l}\text { Investigating the performance of aerial radio } \\
\text { connectivity using simulations }\end{array}$ & $\begin{array}{l}\text { The interference mitigation scheme provided } \\
\text { good potential. }\end{array}$ & $\begin{array}{l}\text { The network performance degraded when it is } \\
\text { required to maintain for higher penetration of } \\
\text { connected aerial vehicles. }\end{array}$ \\
\hline & Ref. [214] & Multiple UAVs/Mobile & Multiple BSs & Rural area & $\begin{array}{l}\text { Feasibility of providing LTE connectivity for } \\
\text { UAVs }\end{array}$ & $\begin{array}{l}\text { Enhanced the LTE connectivity towards UAVS } \\
\text { while protecting the performance of GUs. }\end{array}$ & High altitude UAVs cannot be covered. \\
\hline & Ref. [215] & Multiple UAV-BSs/Mobile & Multiple BSs & Urban area & $\begin{array}{l}\text { Maximizing the cellular coverage by } \\
\text { investigating the optimal altitude and density of } \\
\text { UAV-BSs }\end{array}$ & $\begin{array}{l}\text { Successfully increased the coverage of } \\
\text { UAV-assisted cellular networks while } \\
\text { considering the different physical constraints. }\end{array}$ & $\begin{array}{l}\text { The trajectories of UAV-BSS are not } \\
\text { investigated to optimally increase the coverage } \\
\text { of such networks. }\end{array}$ \\
\hline & Ref. [217] & $\begin{array}{c}\text { Multiple } \\
\text { UAV-BSs/Static/Mobile }\end{array}$ & Multiple BSs & Urban area & $\begin{array}{l}\text { Integrating of UAVs into the next-generation } \\
\text { wireless communication networks }\end{array}$ & $\begin{array}{l}\text { Demonstrated the efficiency of both laser } \\
\text { transmission power and the propagation } \\
\text { attenuation }\end{array}$ & $\begin{array}{l}\text { The security of UAV communication and } \\
\text { interference cancellation are not investigated. }\end{array}$ \\
\hline & Ref. [216] & Multiple UAVs/Mobile & Multiple BSs & Suburban area & $\begin{array}{l}\text { Using UAVs as mobile relays to enhance the } \\
\text { end-to-end ratio based on their optimal } \\
\text { placement }\end{array}$ & $\begin{array}{l}\text { Using the multiple hop strategy is better for } \\
\text { large distances between source and destination. }\end{array}$ & $\begin{array}{l}\text { The optimal placement of UAVs is studied } \\
\text { without considering the impact of the } \\
\text { surrounding environment. }\end{array}$ \\
\hline
\end{tabular}

including antenna beamforming selection, interference mitigation, propagation characteristics, communication range, and channel modeling.

- When adopting one of these techniques, various issues may be raised, such as the degradation of network throughput, the high aerial interference, the restricted coverage, and the negligence of surrounding environments and physical constraints.

- The spectrum sharing and channel access show all their importance and they deserve to be well investigated in order to avoid other problems that may be raised on different sides [218].

\section{Open Research Challenges}

To provide a full connectivity coverage of a given region that is partially covered by ground BSs, it is a mandatory condition to address several problems, such as the required density of UAVs and the backhaul connectivity of UAVs and their GUs. Moreover, in the case when UAV-BSs are deployed, other important issues have to be considered, such as the high mobility of both GUs and UAVs, the energy constraint of UAVs and GUs, the vulnerable frequency when UAV-BSs, GUs, and BSs communicate at the same time, the scalability of the network when new UAVs or GUs join it. As a result, it is imperative to make an accurate analysis of such UAV assistance to traditional cellular networks.

\section{UAV-IOT NETWORKS}

Many cases of IoT networks' deployment have been witnessed in different contributions across the literature in which UAVs are involved to overcome both their restricted capacities and the different constraints that may face. Indeed, different challenges are distinguished in such kind of UAV-IoT coordination, which are listed as follows:
- The inability of IoT devices to communicate with other distant devices due to their restricted coverage caused by their battery-limited capacity [219].

- The limited energy capacity of UAVs to serve IoT devices all the time.

- The high latency induced during the communication between UAVs while serving IoT devices.

- The definition of the appropriate density of UAVs to deploy to optimally serve IoT devices.

- The minimization of energy consumption of IoT devices. To overcome these challenges, different related contributions are proposed in the literature, which are both discussed in the following subsections and briefly summarized in Table IX.

\section{A. Emergency}

In emergency situations or natural disasters, a communication solution has to be deployed to connect the control center and victims in order to provide the evacuation guidance, the rescue assistance, and the situational awareness [220]. Indeed, an emergency communication network comprising UAVs and IoT devices has to be established with the aim to provide the required wireless coverage in order to both connect all victims to the network and facilitate the intervention of rescue teams (c.f., Fig. 16). Several contributions have been proposed in this application.

In the case of an emergency situation, any collected information by UAVs is crucial and needs to be sent quickly and reliably to the relevant services. For instance, in [221] proposed a routing protocol with a bounded delivery delay with the aim to guarantee that rescue information can be delivered in time when a disastrous event happens. This protocol uses three main features: (i) time division packet delivering, (ii) hierarchical UAV clustering, and (iii) real-time estimation of the delivery delay. However, when it comes to communication, 


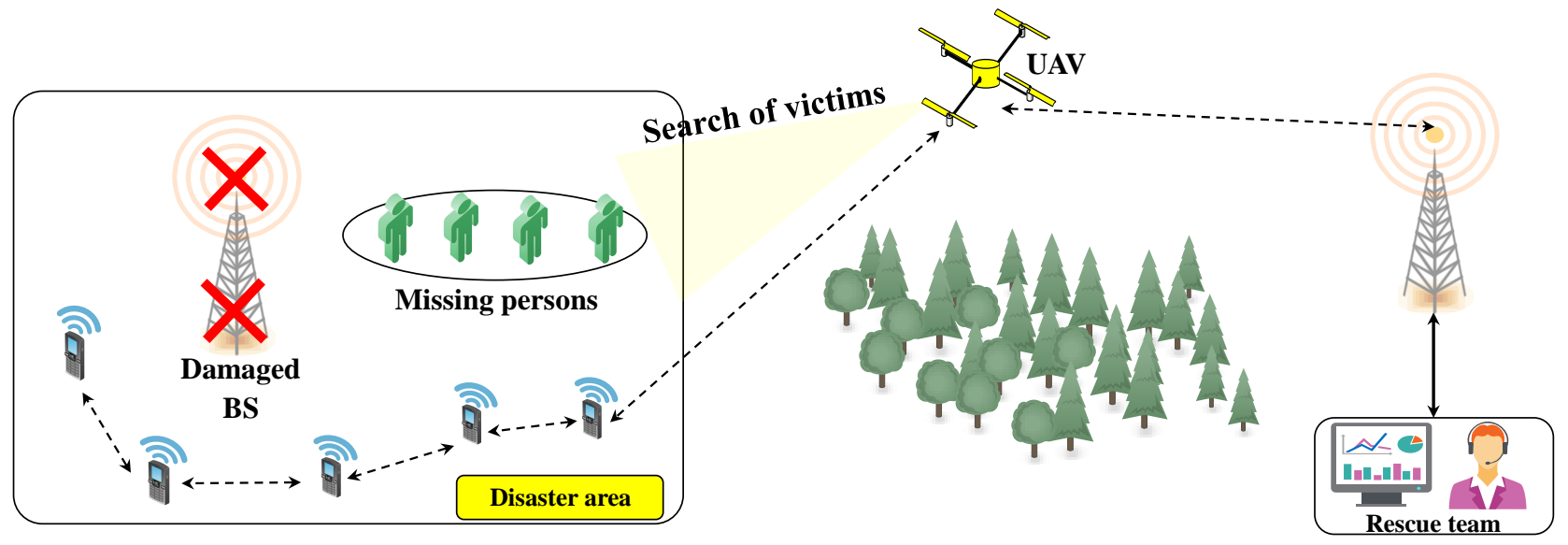

Fig. 16: Emergency in UAV-IoT networks.

the batteries tend to be rapidly exhausted. Thus, the necessity to enhance this protocol with an energy-efficient mechanism. To extend the wireless coverage of UAVs in disaster scenarios, the work in [222] combined a multi-antenna transceiver model and a multi-hop D2D communication to ensure a reliable transmission for IoT networks. This system demonstrates a good network performance in terms of throughput and delivery delay. As a disadvantage, no measures are taken against the energy restriction capacity of UAVs. With the aim of evacuating victims from a disaster event to a safe area, Aljehani et al. [223] proposed a multi-UAV system integrating machineto-machine (M2M) communication for disaster response. Two kinds of missions are carried out by UAVs: (i) Tracking mission and (ii) Scanning mission, which are based on image and high computational processing, respectively. The aim of these two missions is to control UAVs and distinguish valuable data at the same time. Moreover, a real-time evacuation map is made from intercepting images of areas and pedestrians. Nevertheless, the efficiency of this system highly depends on the walking speeds of victims. In the same way, the authors of [224] proposed an evacuation guidance system using Agentbased IoT. As a drawback, this system does not have the ability to both perform the evacuation guidance based on unexpected events and neglect the cooperation of UAVs.

Findings: The learned lessons are outlined as follows:

- UAV-IoT coordination is crucial and vital is some emergency situations. Indeed, a robust network model needs to be conceived while considering the delay-sensitive nature of this kind of applications. Moreover, important measures have to be taken against packet losses.

- The coverage must be extended to optimally scan the whole disaster region. This can be done by defining the appropriate density of UAVs to be deployed [225].

- The energy efficiency of UAVs has to be considered in such kinds of applications.

\section{B. Smart Cities}

Different applications involving UAVs and IoT devices are proposed to perform different missions in a city environment with the aim to enhance the quality of citizens' life. For instance, these applications can manage different fields, such as transportation systems [226], agriculture [227], emergencies [228], health-care [229], smart home [230], and others (c.f., Fig. 17). This management requires multiple interconnected mobile or stationary devices in order to provide efficient infrastructure and services at a reduced cost. A lot of research work has been done in this way.

In [231], an air quality monitor system is proposed using UAVs and ground nodes, which are all equipped with sensors. This system comprises four different layers: (i) the presentation layer is used for providing a Graphical User Interface (GUI), (ii) the processing layer is used to process the gathered data, (iii) the transmission layer is used for fullduplex communications, and (iv) the sensing layer is used for collecting data. Moreover, to support these layers, three techniques are adopted to enhance the latency of data uploading, to optimize the deployment of nodes for maximizing collected data, and to balance the energy consumption among nodes. However, the coverage provided by UAVs is restricted only for a limited surface. While in [232], the authors proposed an intelligent IoT platform using UAVs to provide various smart city services, such as smart transportation systems, smart home, smart UAV, and other future services according to the consumer needs. This platform comprises four physical components: (i) the UAV, (ii) the client, (iii) the operator, and (iv) the cloud server. Moreover, this platform is enabled based on $\mathrm{M} 2 \mathrm{M}$ communications and the multi-agent system concept. Nevertheless, the energy consumption consideration is missing in this system. Another UAV-IoT network is considered by the authors of [233] in which a UAV-based IoT platform used for data offloading in mobile edge computing while enhancing the energy consumption of UAVs is proposed. Nevertheless, this platform did not consider the case of energy consumption during simultaneous applications. Moreover, the use of one Mobile Edge Computing (MEC) node does not support the required computational performance. To increase the data collection efficiency, in [234], the authors optimized the transmission power and duration of all devices of UAVaided data collection for maritime IoT. In addition, UAVs are effectively deployed to achieve flexible and dynamic coverage 


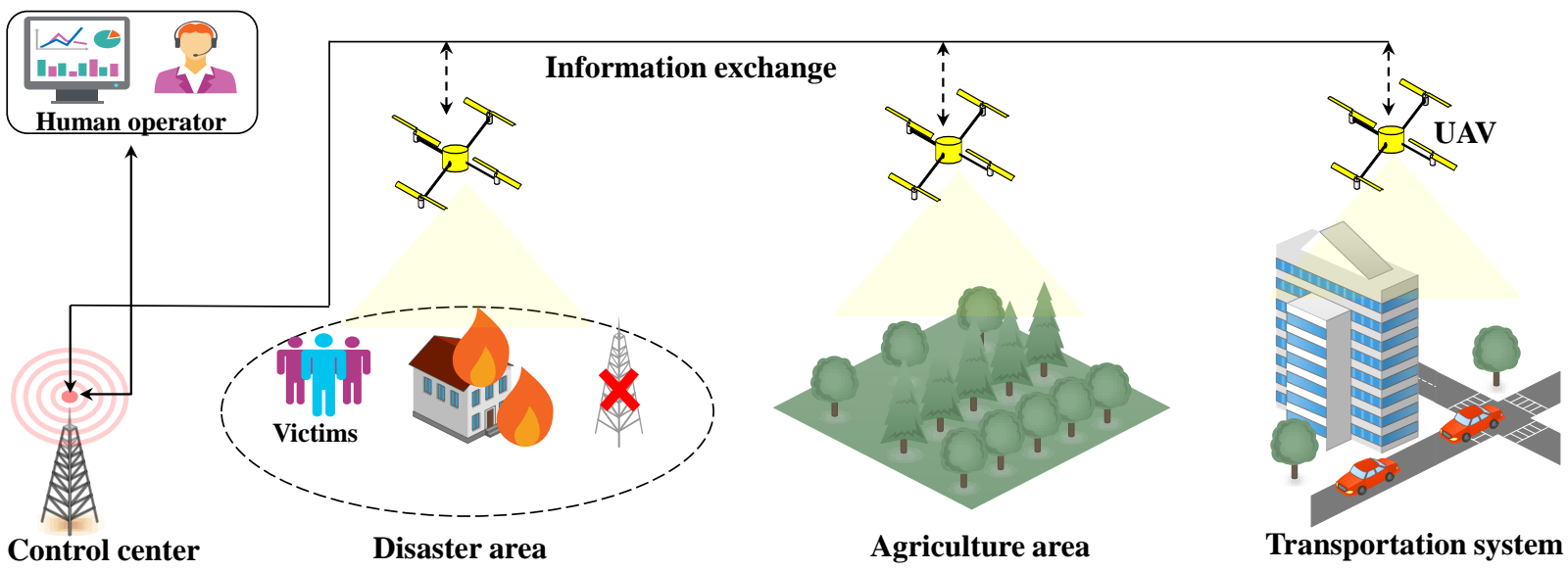

Fig. 17: Smart cities in UAV-IoT networks.

of the ground network. As an inconvenience, the deployment of a single UAV for data collection may constitute a crucial issue.

Findings: UAV-IoT coordination in the context of a smart city is becoming a hot topic, and numerous lessons learned can be extracted from this subsection as follows:

- A robust and reliable architecture has to be carefully studied and simulated before putting it on the field. Indeed, the different communicating entities, the networking model, and the task assignment have to be all wellinvestigated.

- Since there is a huge amount of data can be generated, a robust computational model can be used by exploiting the existing infrastructures. For instance, a cloud computing platform can be easily defined by exploiting ground BSs.

- Multiple challenges can be also distinguished from this UAV-IoT coordination, such as energy consumption, the trajectory of UAVs, and the required density of UAVs to be deployed.

\section{Industry and Agriculture}

Due to their fast deployment, their controllable flexibility, their easy programmability, and their capacity to be deployed in complex environments, UAVs are considered as the most appropriate devices to embed visual inspection and communication devices in different sectors, such as industrial fields [235], [236] and agricultural areas [237]-[239]. Moreover, UAVs can intercept data and images from both IoT devices and the field, respectively. All this information is sent to a control entity to manage the production and to ensure the right functionality of the different equipment (c.f., Fig. 18). Multiple contributions involving UAVs and IoT devices have been proposed, which are targeted especially the two different production sectors.

Aiming at collecting data in an agricultural area, the work in [240] proposed FarmBeats. This system is an IoT platform dedicated for agricultural purposes. This architecture enables data collection from a variety of sensors, cameras, and UAVs, in order to provide automated water intervention. This can ensure that services and information are available offline and in the existing cloud. However, the complexity of this system is considered as its main drawback. Similarly, the authors of [241] presented a UAV-aided solution based on IoT concepts to provide the necessary improvement of crop quality in the agriculture field. However, the use of a single UAV has been always a crucial issue due to the risk that can cause to the whole network in the case of its failure. In the case of indoor monitoring in industrial environments, in [242], a safe navigation system for micro UAVs based on WSNs has been proposed. This system is deployed in the industrial IoT environments with the aim to detect dynamic and static obstacles. Three different components have been considered in this system: (i) UAVs equipped with tracking controller, (ii) a central controller, and (iii) a group of sensors to detect any kind of obstacles. Based on all this information, each UAV can generate a safe path that is tracked to its destination. However, the adopted centralized architecture is not efficient in case of failure of the central controller. Always in the industrial environments, Zhou et al. [243] investigated how to deploy energy efficient Industrial Internet of UAVs (IIoUAVs) for power line inspection in smart grid. The decreasing of energy consumption is formulated as a joint optimization, which involves both large-scale optimization of time, such as frequency regulation, speed control, trajectory scheduling, and small-scale time optimization, such as relay selection and power allocation. As a drawback, the mobility optimization of UAVs for collision avoidance is not considered. Scilimati et al. [244] proposed an IoT-enabled robotic system using UAVs to interact with the surrounding environment (i.e., monitoring) and collect data from sensors. For this purpose, this system composed of an IoT device connected to the UAV that is monitoring the specified area, where the IoT network is deployed to gather data from the environment. The specific use case of this system does not allow it to be adapted to outdoor environments.

Findings: The exploitation of UAVs in the agricultural and industrial sectors faces many challenges. In this subsection, many lessons are learned and can be summarized as follows:

- Due to these specific use cases, a complex architecture is distinguished at each proposed application. This is why 


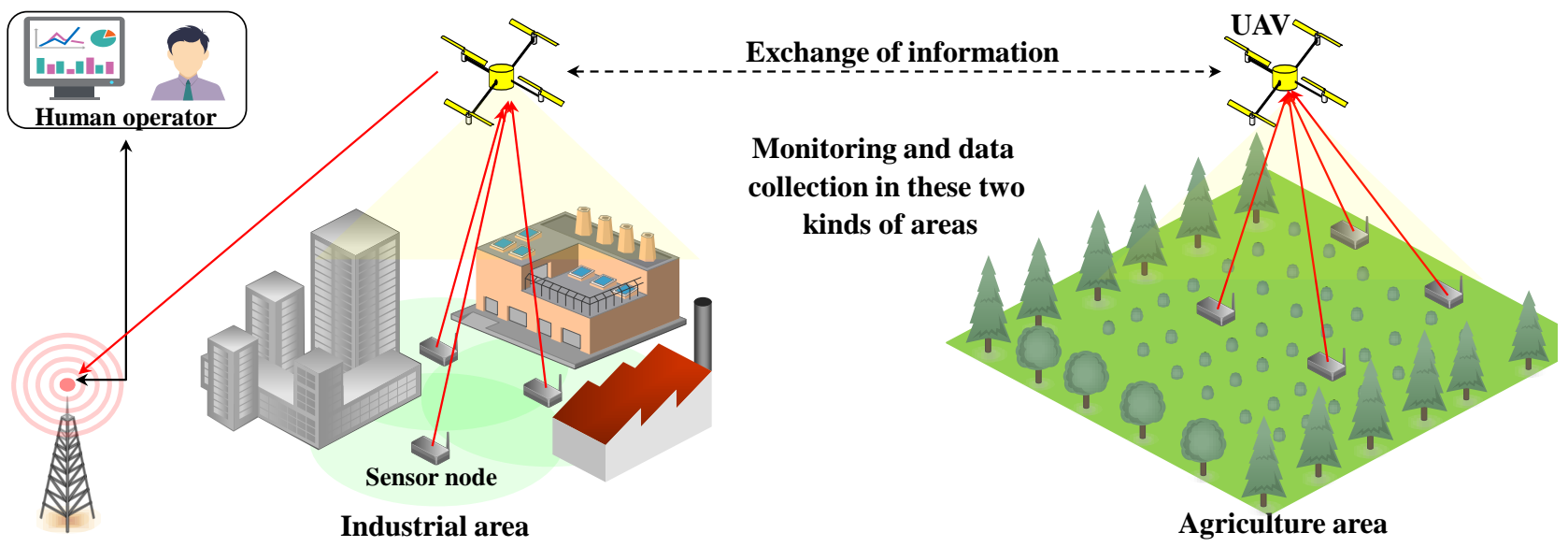

Fig. 18: Industry \& Agriculture in UAV-IoT networks.

the flexibility of the architecture is of major concerns.

- The data collection is used in the majority of contributions proposed for this scope. Therefore, all challenges resulting in this kind of applications have to be considered.

- Due to the restricted areas that need to be covered, some parameters, such as speed control, trajectory scheduling, and obstacle avoidance, have to be well regulated.

\section{WSN and Monitoring}

There is a case when IoT devices play an exclusive role of sensors with the objective to perform remote sensing of a specified environment [245]. UAVs can be deployed in such environments to help IoT devices to go beyond their limits and perform their assigned tasks efficiently (c.f., Fig. 19). Moreover, many monitoring use cases have been distinguished in UAV-IoT networks, such as gathering collected images from IoT devices, monitoring existing UAVs to avoid collisions, and monitoring existing IoT infrastructure [246]. Indeed, the major tasks of UAVs are to collect IoT data and to relay them to the adequate entity while considering the different constraints that may arise. This offers a global knowledge and a smooth operating of such networks. Several UAV-WSN coordination and monitoring contributions are proposed across the literature.

More specifically, the work in [247] proposed both a coalition model and an algorithm derived from basic geometry dedicated for remote sensing missions using UAVs. By planning paths of equal length, it supposed that all UAVs are moving at the same and constant speed in order to ensure simultaneous arrival. This can perform remote sensing and monitoring in a timely manner, thus decreasing the mission time. However, the environmental conditions are not considered, which can impact the mobility of UAVs. Another kind of remote sensing is accomplished by the work in [248], which proposed an aerial sensing framework over an IoT infrastructure. To provide a navigation ability to remote users in a given area, this framework uses the UAV multimedia perception system. This can be done by using virtual reality (VR) and augmented reality (AR) devices over the intercepted data. As a drawback, a high delivery delay and bandwidth are required due to a large amount of data that can be sensed. Over a given area, UAVs can also support IoT services. For instance, Gaur et al. [249] proposed a scheme connecting IoT with UAVs to disseminate sensor data to the cloud infrastructures. This system provides effective data management by efficiently collecting, filtering, and delivering data on demand for cloud services. Moreover, a vertical handover technique is also adopted between the different modes of communication in order to enhance reliability and minimize cost. However, the intermittent connectivity of the UAV network and the non-balanced energy consumption of UAVs constitute the main drawback of this system. To overcome these drawbacks, Yoo et al. [250] investigated the flying path optimization for UAV-assisted IoT sensor networks based on a location-aware multi-layer map. Various functions have been considered, such as flight time, energy consumption, and sensor density. Moreover, the links between IoT devices and UAVs have been facilitated using some features of genetic algorithms. The major drawback of this system is the limited and little surface that can be covered by UAVs.

As for monitoring purpose, UAVs can be considered as the appropriate candidates to perform this kind of task. Aiming at this, Bushnaq et al. [251] proposed an aerial data collection scheme over a delimited region using a UAV. Instead of routing data through several other relays, the UAV has the ability to fly over the area of interest and collect the required data. In particular, the area is divided into multiple sub-regions on which the UAV flies over to gather samples from selected nodes. To this end, an optimization problem is formulated to define the optimal number sub-regions, the hovering locations, the hovering time at each location and the path traveled between hovering locations so that an average number of samples are gathered from the area in a minimum amount of time. In [252], an approach for round-the-clock surveillance supported by UAVs. This approach provided an IoT-based automated landing system with constant monitoring of installations. The latter system is composed of four kinds of software: (i) the console server, (ii) communication protocol for UAVs, (iii) landing zone firmware to control both wireless charging and active gripper, and (iv) application of mobile 
users intercepting the UAVs video from the server in real-time. However, environmental conditions can distort the functioning of this system. When the purpose of monitoring is dedicated to track amateur UAVs, Ding et al. [253] proposed the incorporation of UAV communication systems and cognitive radio, which is referred to as cognitive UAV architectures. This is beneficial to allow the coexistence of UAVs with ground mobile devices and amateur UAVs functioning in the same frequency band. However, the deployed architecture is very complex and not cost-effective. In order to reduce the movements while accomplishing a near-optimal monitoring, Kim et al. [254] proposed a monitoring model dedicated to multi-domain IoT environment. This model is assisted by reinforcing barriers with collision-avoidance using UAVs. In reality, the energy consumption of UAVs is not considered, which affect the right functionality of this approach.
Findings: Using WSN for monitoring purpose has shown all its importance. Moreover, a number of lessons are learned during our study of this kind of applications, which can be summarized as follows:

- Remote sensing using communications and surveillance using a camera are the most used techniques to perform UAV-WSN monitoring.

- Since this kind of applications can be deployed in outdoor environments, the surrounding conditions, such as bad weathers, obstacles, complex areas (e.g., mountains) have to be considered.

- Many technical challenges can be distinguished, such as the allocated bandwidth, the limited processing capabilities, the intermittent connectivity of the UAV network, and the energy consumption of UAVs and ground nodes. All these challenges need a deep study to design a robust monitoring scheme.

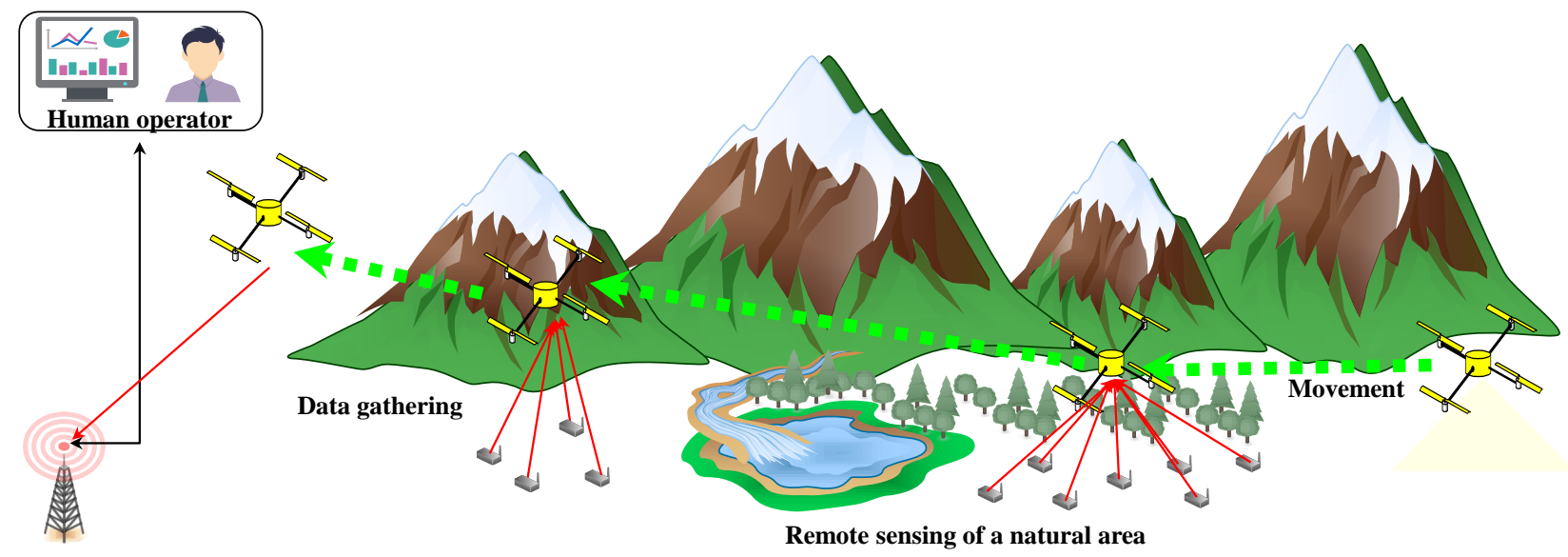

Fig. 19: WSN in UAV-IoT networks.

TABLE IX: Summary of contributions to UAV-IoT networks.

\begin{tabular}{|c|c|c|c|c|c|c|c|}
\hline & \multirow{3}{*}{$\begin{array}{c}\text { Density and Mobility of } \\
\text { UAVs }\end{array}$} & \multirow{3}{*}{$\begin{array}{c}\begin{array}{c}\text { Types of ground } \\
\text { nodes }\end{array} \\
\text { Multiple sensors }\end{array}$} & \multirow{3}{*}{$\begin{array}{c}\begin{array}{c}\text { Deployment } \\
\text { environment }\end{array} \\
\text { Disaster area }\end{array}$} & \multirow[b]{2}{*}{ Objective } & \multirow[b]{2}{*}{ Advantage } & \multirow[b]{2}{*}{ Drawback } \\
\hline & & & & & & & \\
\hline \multirow[b]{4}{*}{ Emergency } & Ref. [221] & & & & $\begin{array}{l}\text { Performing reliable and on-time transmission } \\
\text { of rescue transmission }\end{array}$ & $\begin{array}{l}\text { Improved the overall system in terms of } \\
\text { delivery ratio and delay. }\end{array}$ & $\begin{array}{l}\text { The energy consumption of UAVs is not } \\
\text { considered. }\end{array}$ \\
\hline & Ref. [222] & Multiple UAV-BSs/Mobile & IoT devices & Disaster area & $\begin{array}{l}\text { Providing emergency coverage for IoT devices } \\
\text { on the ground }\end{array}$ & $\begin{array}{l}\text { Demonstrated a good performance of the } \\
\text { system in place. }\end{array}$ & $\begin{array}{l}\text { Did not save energy for the limited batteries of } \\
\text { UAV-BSs. }\end{array}$ \\
\hline & Ref. [223] & Multiple UAVs/Mobile & IoT devices & Disaster area & $\begin{array}{l}\text { Establishing alternative routes for evacuating } \\
\text { victims }\end{array}$ & $\begin{array}{l}\text { Helped victims to be evacuated efficiently to a } \\
\text { safe area. }\end{array}$ & Depended on the walking speed of victims. \\
\hline & Ref. [224] & Multiple UAVs/Mobile & $\begin{array}{l}\text { Single UGV/Multiple } \\
\text { IoT devices }\end{array}$ & Disaster area & $\begin{array}{l}\text { Defining an evacuation plan based on the } \\
\text { situation of the environment }\end{array}$ & $\begin{array}{l}\text { Provided satisfactory performance of this } \\
\text { system in terms of evacuation routes and } \\
\text { network performance. }\end{array}$ & $\begin{array}{l}\text { Did not perform evacuation guidance under } \\
\text { unexpected events and neglected the } \\
\text { cooperation of UAVs. }\end{array}$ \\
\hline \multirow{4}{*}{ Smart cities } & Ref. [231] & Single UAV/Mobile & Multiple BSs & Urban area & $\begin{array}{l}\text { Monitoring air quality based on aerial and } \\
\text { ground sensing }\end{array}$ & $\begin{array}{l}\text { Aerial and Ground sensor devices are used to } \\
\text { balance between the energy consumption and } \\
\text { the data accuracy. }\end{array}$ & $\begin{array}{l}\text { Cannot provide a complete service in a wide } \\
\text { urban area. }\end{array}$ \\
\hline & Ref. [232] & Multiple UAVs/Mobile & $\begin{array}{c}\begin{array}{c}\text { Multiple BSs/IITT } \\
\text { devices }\end{array} \\
\end{array}$ & $\begin{array}{c}\text { Urban/Agricultural } \\
\text { area }\end{array}$ & $\begin{array}{l}\text { Solving multiple issues within the smart city } \\
\text { implementation }\end{array}$ & $\begin{array}{l}\text { Provided a significant benefit of reduced cost } \\
\text { in different services and sectors. }\end{array}$ & $\begin{array}{l}\text { The restricted-energy capacity of UAVs is still } \\
\text { an open issue. }\end{array}$ \\
\hline & Ref. [233] & Multiple UAVs/Mobile & $\begin{array}{l}\text { Multiple IoT } \\
\text { devices/BSs }\end{array}$ & Urban area & Transmitting IoT services from high altitudes & $\begin{array}{l}\text { Demonstrated a high computation offloading } \\
\text { while saving energy consumption and } \\
\text { improving the response time. }\end{array}$ & $\begin{array}{l}\text { The use of one MEC node for supporting } \\
\text { computational performance. }\end{array}$ \\
\hline & Ref. [234] & Single UAV/Mobile & Multiple IoT devices & Urban area & $\begin{array}{l}\text { Investigating the potential gain of UAV-assisted } \\
\text { data gathering over IoT devices }\end{array}$ & $\begin{array}{l}\text { Adapted well to the rigorous energy constraints } \\
\text { as well as the large-scale of the IoT network. }\end{array}$ & $\begin{array}{l}\text { The deployment of a single UAV can be an } \\
\text { issue to cover a wide geographical area. }\end{array}$ \\
\hline \multirow{5}{*}{ Industry \& Agriculture } & Ref. [240] & Multiple UAVs/Mobile & $\begin{array}{l}\text { Multiple IoT } \\
\text { devices/BS }\end{array}$ & Agricultural area & $\begin{array}{l}\text { Agriculture IoT platform enabling seamless } \\
\text { data collection from various sensors, cameras } \\
\text { and UAVs. }\end{array}$ & $\begin{array}{l}\text { Ensuring that services are available offline and } \\
\text { in the Cloud. }\end{array}$ & The complexity of the platform design. \\
\hline & Ref. [242] & Multiple UAVs/Mobile & Multiple sensors & Industrial area & $\begin{array}{l}\text { Detecting static and dynamic obstacles in an } \\
\text { indoor industrial environment }\end{array}$ & $\begin{array}{l}\text { Provided a good monitoring performance in a } \\
\text { large indoor industrial area. }\end{array}$ & $\begin{array}{l}\text { A centralized architecture is adopted, which } \\
\text { can fail at any time. }\end{array}$ \\
\hline & Ref. [243] & Multiple UAVs/Mobile & Multiple BSs & Industrial area & Power line monitoring in smart grid & $\begin{array}{l}\text { Demonstrated its energy consumption } \\
\text { efficiency. }\end{array}$ & $\begin{array}{l}\text { The trajectories of UAVs are not optimized and } \\
\text { collision avoidance is not considered. }\end{array}$ \\
\hline & Ref. [241] & Single UAV/Mobile & Multiple sensors & Agricultural area & Monitoring crop management & Collected data required in precision agriculture. & $\begin{array}{l}\text { The use of a single UAV to monitor a wide } \\
\text { agricultural field. }\end{array}$ \\
\hline & Ref. [244] & Single UAV/Mobile & Multiple IoT devices & Industrial area & UAV-assisted environmental monitoring & $\begin{array}{l}\text { Provided a good performance of data } \\
\text { collection. }\end{array}$ & Cannot be adapted in outdoor environments. \\
\hline \multirow{8}{*}{ WSN \& Monitoring } & Ref. [247] & Multiple UAVs/Mobile & None & Urban area & Monitoring and achieving remote sensing & Decreased the mission time. & $\begin{array}{l}\text { Collision avoidance and environmental } \\
\text { conditions are not considered. }\end{array}$ \\
\hline & Ref. [248] & Multiple UAVs/Mobile & Single BS & Rural area & $\begin{array}{l}\text { Delivering multiple VR/AR immersive } \\
\text { communication sessions for remote users based } \\
\text { on UAV IoT aerial sensing }\end{array}$ & $\begin{array}{l}\text { Increased the end-to-end performance across } \\
\text { the VR/AR sessions delivered to the users. }\end{array}$ & $\begin{array}{l}\text { Required both high bandwidth and an } \\
\text { important delay due to a large amount of } \\
\text { sensed data. }\end{array}$ \\
\hline & Ref. [249] & Multiple UAVs/Mobile & $\begin{array}{c}\text { Multiple IoT } \\
\text { devices/sensors }\end{array}$ & Not specified & Delivering IoT services using UAVs & $\begin{array}{l}\text { The seamless handover is addressed between } \\
\text { Wi-Fi and satellite network. }\end{array}$ & $\begin{array}{l}\text { The high energy consumption of UAVs and the } \\
\text { intermittent connectivity of the UAV network. }\end{array}$ \\
\hline & Ref. [250] & Single UAV/Mobile & $\begin{array}{c}\text { Multiple IoT } \\
\text { devices/sensors }\end{array}$ & Not specified & $\begin{array}{l}\text { Defining a near optimal flying path for the } \\
\text { UAV while considering different factors }\end{array}$ & $\begin{array}{l}\text { Derived the desired optimum path while } \\
\text { satisfying the existing constraints }\end{array}$ & $\begin{array}{l}\text { The energy restriction of the UAV did not } \\
\text { allow it to cover a wide area. }\end{array}$ \\
\hline & Ref. [251] & Single UAV/Mobile & Multiple IoT devices & Not specified & $\begin{array}{l}\text { Aerial data gathering from a finite spatial field } \\
\text { via a UAV }\end{array}$ & $\begin{array}{l}\text { Minimized the traveling and the hovering time } \\
\text { during the data collection. }\end{array}$ & $\begin{array}{l}\text { Considered only a small region for data } \\
\text { aggregation. }\end{array}$ \\
\hline & Ref. [252] & Single UAV/Mobile & Single BS & Military area & Monitoring using a UAV & $\begin{array}{l}\text { Validated a robust landing system with } \\
\text { charging purpose. }\end{array}$ & Environmental disturbances are not considered. \\
\hline & Ref. [253] & Multiple UAVs/Mobile & $\begin{array}{c}\text { Multiple } \\
\text { sensors/Single BS }\end{array}$ & Not specified & Monitoring amateur drone & Efficiently detected amateur drones. & $\begin{array}{l}\text { A complex architecture and not cost effective } \\
\text { infrastructure. }\end{array}$ \\
\hline & Ref. [254] & Multiple UAVs/Mobile & Multiple IoT devices & Harsh area & $\begin{array}{l}\text { Reducing the movement of UAVs under a } \\
\text { collision-avoidance condition }\end{array}$ & $\begin{array}{l}\text { Provided a good network performance in terms } \\
\text { of data sensing. }\end{array}$ & $\begin{array}{l}\text { There is no measurement taken against the } \\
\text { energy restriction of UAVs. }\end{array}$ \\
\hline
\end{tabular}




\section{Open Research Challenges}

Different IoT devices can be integrated with UAVs to enable several IoT applications with the aim to exploit networking, computing, and caching technologies. Nevertheless, additional features can create unexpected issues that cannot be addressed through the classical approaches designed for low-rate IoT systems, such as the inefficient data collection, the high energy consumption of UAVs and IoT devices, the environmental disturbances. As a consequence, robust schemes will need to be designed for providing efficient services to users while keeping a certain level of networking performance.

\section{UAV Disaster MANAGEMENT}

The disaster management is decomposed of three steps: (i) Pre-disaster supervision, (ii) Search and Rescue (SAR), and (iii) Recovery (Post-disaster assistance) [255]. In the first step, UAVs play a key role to allow emergency teams reaching inaccessible areas and discovering them based on captured information. Moreover, UAVs support early detection of disasters using real-time monitoring so that the effect of the disaster can be efficiently mitigated [256]. In the second step, when a disaster is unavoidable (e.g., Earthquake), UAVs can access and respond effectively to emergency requests by monitoring the target area, tracking possible missing victims, and providing time-critical rescue aids [257]. The last step allows UAVs to put in place an emergency communication infrastructure to support recovery and to help rescue teams to be deployed accurately in the area of interest [258]. All these steps are illustrated in Fig. 20. However, there exist some challenges remain and some have grown insignificance. In the following, we list the major ones:

- Defining the adequate number of UAVs to be deployed to efficiently perform the rescue missions in a timely manner.

- Ensuring robust connectivity between UAVs and the central controller in order to reliably exchange crucial information.

- Efficiently replacing damaged ground infrastructures by using UAVs.
- Jointly optimizing the placement and mobility of UAVs according to the requirements of the rescue missions.

As a consequence, several contributions involving UAVs have been developed in the last five years, which are summarized in Table X.

\section{A. Pre-Disaster Supervision}

The aerial assessment provides the most effective and timely knowledge of the situation in a given area [259]. This is because UAVs have the ability to travel quickly to affected areas and to easily take pictures and videos on the current situation. Indeed, UAV systems were required to be deployed a long time ago to anticipate any eventual disasters. In addition, other networks can be hybrid-connected with UAVs, such as IoT networks or WSNs to ensure that any information would be intercepted and processed in time.

In order to perform a real-time assessment of disaster events, Erdelj et al. [260] proposed a hybrid system incorporating the sensing capacities and the visual capturing abilities of WSNs and multi-UAVs systems, respectively. Moreover, WSNs act as detection systems that trigger the launch of UAVs following a disastrous event. With this approach, the density of UAVs that required to be deployed is not defined accurately. As a further advance, the authors of [261] proposed a hybrid system composed of WSN and UAVs dedicated to disaster management. This system uses inundation monitoring and alarm technology by incorporating smartphone data with sensors for improved situational awareness. Moreover, a networking model is used for relaying information between the different nodes, but their energy constraints are not considered. To monitor different disaster areas, in [262], a system deploying a multi-UAV network in civilian monitoring applications is proposed. In this system, different kinds of UAVs are used, such as micro UAVs, Quadcopters, and fixed-wing UAVs, which are all equipped with special sensors to monitor different natural disasters, such as earthquakes, floods, and volcanoes. Moreover, collected data is processed by modules exclusively designed for this purpose. As a drawback, the complexity of the adopted architecture during the processing of collected data is very high. In contrast, Asadpour et al. [263] demonstrated an experiment
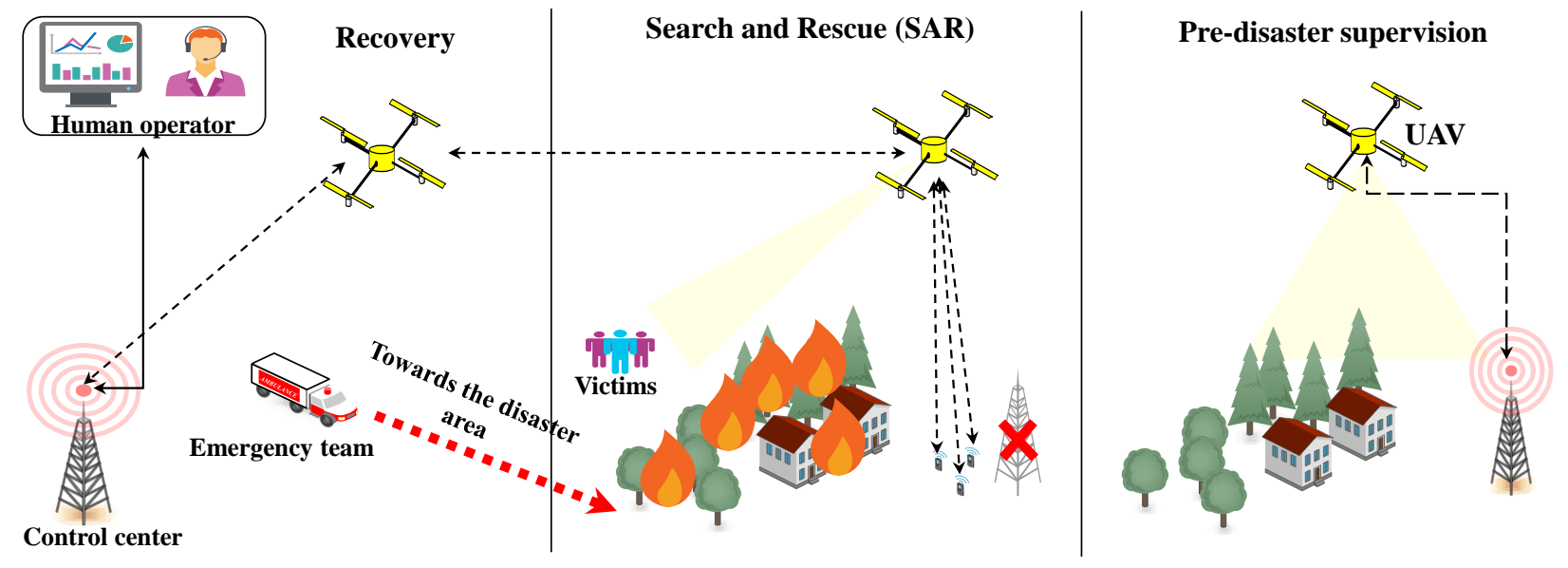

Fig. 20: UAV-assisted disaster management. 
of the feasibility of UAV communication in disaster scenarios. To find victims, two different use cases are distinguished for UAVs. First, UAVs establish a wireless link by intercepting beacons from smartphones of victims, where UAVs do not require to have a LoS with the devices of victims. Second, UAVs provide aerial images in real-time, which are relayed using the existing multi-hop UAV network. Consequently, this system is considered to be based on a flexible network that can be adapted to any kinds of rescue applications.

Findings: Three main lessons can be learned from the predisaster management using UAVs as follows:

- To ensure a good preparedness towards any disaster events, an important number of measures should be taken into account, such as the required density of UAVs to monitor the area, a robust networking model to relay information to the closest human operator, and a high processing and storage performance needs to be guaranteed.

- The flexibility and ease of installation of the designed architectures which can be adapted to any kinds of situations.

- The environmental conditions have to be considered to ensure and transmit accurate information defining the status of the area that is supervised.

\section{B. Search and Rescue}

In SAR missions, the main challenge for researchers is to decide which UAVs are allocated to search tasks and which UAVs are allocated to rescue tasks within a certain time frame [264]. The UAVs in charge of search tasks can use two techniques: (i) Monitoring using mounted cameras and (ii) Search using embedded wireless communications devices on both UAVs and missing victims. Moreover, other techniques are jointly optimized to allow successfully achieving those tasks, such as the energy-efficiency [265], position and path optimization [266], and resource allocation [267].

Based on the assumption of the unlimited energy capacity of UAVs, in [268], a multi-objective path planning using multiple UAVs is proposed, which is dedicated for Search and Rescue (SAR) missions. A given mission is performed by following two steps: (i) a search step performed by using cooperative UAVs to both detect targets and relay this information to BS and (ii) a time-to-complete step carried out by a genetic algorithm minimizing both the discovery time of targets and the duration to set up a communication path. As a result, this system reduces significantly the mission completion and communication recovery times. Silvagni et al. [269] proposed a UAV application for SAR missions during avalanches in the mountains. This work includes a body detection technique by using avalanche sensors equipped onto UAVs to communicate with beacon transmitters carried by survivors. Moreover, UAVs are equipped with different kinds of cameras, such as normal day cameras and thermal night cameras with the aim to make the detection more efficient. As a drawback, UAVs are not able to track victims that are not visible. Based on a different technique, Kurdi et al. [270] proposed a bio-inspired algorithm based on locusts for task allocation in multi-UAVs systems during search and rescue missions. Two different phases are conducted by this algorithm: (i) the search phase and (ii) the rescue phase, which are assigned to two different groups of UAVs collaborating with each other and reporting back any updates to the BS in order to efficiently achieve the search and rescue objectives. As an advantage, the communication with BS is not required for all UAVs. However, a deep study needs to be performed to define accurately the number of UAVs to be deployed. By gathering aerial images in a realtime manner, Sun et al. [271] proposed a method of camerabased target detection and positioning system for search and rescue missions. This method is incorporated in UAVs in order to be able to perform the identification of objects and their geographical coordinates in a real-time. Moreover, UAVs send the collected images and their locations to the ground BS. Since a huge amount of data can be collected, UAVs need more computational and storage capacities. To top it off, Scherer et al. [272] proposed a modular architecture using a set of autonomous UAVs for search and rescue missions. Based on the mission requirements, this system incorporates ground image collection, GPS-based navigation, payload delivery, and different autonomy levels, all implemented in a lowcost platform. Moreover, this modular architecture provides certain fault-tolerance capabilities and some exciting future expansion opportunities. The LoS between UAVs and BSs are required to avoid any communication loss between them. Oubbati et al. [273] proposed a framework that leverages cooperative UAVs to facilitate the intervention of rescue teams on board emergency vehicles in the accident zones in urban environments. Indeed, UAVs have the ability to detect any accidents in road segments, inform the rescue teams about their exact locations, and plot the quickest path to intervene. However, there is a need to deploy an important of UAVs to cover all road segments, which is not a cost-effective solution.

Findings: Search and rescue missions show many challenging issues that are summarized as follows:

- The completion time of SAR missions is a critical parameter because in the majority of cases it is a question of human lives to rescue in a timely manner.

- Replacing damaged infrastructures by UAV-BSs is an important part of SAR missions in order to connect missing victims, detect their locations, and thus facilitate their rescue.

- The loss of communications between UAVs, the human operator, and the victims has to be avoided by studying robust mechanisms of communication.

\section{Recovery}

Generally, after a disaster, an important part of the communication infrastructure becomes damaged and dysfunctional [274]. In this case, the rapid deployment of a secondary communication infrastructure becomes a mandatory condition. Indeed, due to their flexibility and adjustable mobility, UAVs are considered as the best solution to provide emergency connectivity to rescue teams, to victims, and to control servers. This allows coordinating emergency responses and aid efforts to successfully perform SAR missions. 
TABLE X: Summary of contributions to UAV disaster management.

\begin{tabular}{|c|c|c|c|c|c|c|c|}
\hline & & $\begin{array}{c}\text { Density and Mobility of } \\
\text { UAVs }\end{array}$ & $\begin{array}{c}\begin{array}{c}\text { Types of ground } \\
\text { nodes }\end{array} \\
\end{array}$ & $\begin{array}{l}\text { Deployment } \\
\text { environment }\end{array}$ & Objective & Advantage & Drawback \\
\hline \multirow{4}{*}{ Pre-disaster optimization } & Ref. [260] & Multiple UAVs/Mobile & Multiple sensors & Disaster area & $\begin{array}{l}\text { Defining a complete disaster } \\
\text { management system }\end{array}$ & $\begin{array}{l}\text { Identified the issues and challenges } \\
\text { related to disaster management. }\end{array}$ & $\begin{array}{l}\text { The exact number of UAVs to be } \\
\text { deployed is not defined. }\end{array}$ \\
\hline & Ref. [261] & Multiple UAVs/Mobile & Multiple sensors/BSs & Disaster area & $\begin{array}{l}\text { networking assistance first } \\
\text { response to disaster management }\end{array}$ & $\begin{array}{l}\text { Designed a networking model for } \\
\text { relaying information between } \\
\text { different nodes. }\end{array}$ & $\begin{array}{l}\text { The energy consumption of UAVs } \\
\text { is not considered. }\end{array}$ \\
\hline & Ref. [262] & Multiple UAVs/Mobile & Multiple BSs & Disaster area & $\begin{array}{l}\text { Monitoring different natural } \\
\text { disaster areas }\end{array}$ & $\begin{array}{l}\text { Accomplished the surveillance } \\
\text { mission in an efficient way. }\end{array}$ & $\begin{array}{l}\text { The complexity of adopted } \\
\text { architecture during the processing } \\
\text { of intercepted data. }\end{array}$ \\
\hline & Ref. [263] & Multiple UAVs/Mobile & Single BS & Forest area & $\begin{array}{l}\text { Creating an ad hoc multihop } \\
\text { network of UAVs to reconnect } \\
\text { isolated smart-phones. }\end{array}$ & $\begin{array}{l}\text { Providing a flexible network } \\
\text { supporting rescue applications. }\end{array}$ & $\begin{array}{l}\text { The unlimited flight duration of } \\
\text { UAVs without considering their } \\
\text { energy restriction. }\end{array}$ \\
\hline \multirow{6}{*}{ Search \& Rescue } & Ref. [268] & Multiple UAVs/Mobile & Single BS & Disaster area & $\begin{array}{l}\text { Minimizing the mission completion } \\
\text { time, including the search task } \\
\text { time and the communication } \\
\text { recovery time }\end{array}$ & $\begin{array}{l}\text { Considerably reduced the SAR } \\
\text { mission time. }\end{array}$ & $\begin{array}{l}\text { Any restriction is assumed about } \\
\text { the energy consumption of UAVs. }\end{array}$ \\
\hline & Ref. [269] & Single UAV/Mobile & Single BS & Mountainous area & $\begin{array}{l}\text { searching and rescuing of missing } \\
\text { victims on snow and in woods }\end{array}$ & $\begin{array}{l}\text { Obtained a flexible movement over } \\
\text { the mountains without provoking } \\
\text { other avalanches. }\end{array}$ & $\begin{array}{l}\text { Did not function correctly when } \\
\text { the victims are fully covered by } \\
\text { snow. }\end{array}$ \\
\hline & Ref. [270] & Multiple UAVs/Mobile & Single BS & Disaster area & $\begin{array}{l}\text { Allocating tasks in multi-UAV } \\
\text { SAR mission using a bio-inspired } \\
\text { algorithm }\end{array}$ & $\begin{array}{l}\text { The communication with the BS is } \\
\text { not required for UAVs during the } \\
\text { mission. }\end{array}$ & $\begin{array}{l}\text { The optimal number of UAVs is } \\
\text { not studied. }\end{array}$ \\
\hline & Ref. [271] & Single UAV/Mobile & Single BS & Disaster area & $\begin{array}{l}\text { Identifying target in real-time and } \\
\text { gathering aerial images for further } \\
\text { mapping purposes }\end{array}$ & $\begin{array}{l}\text { Demonstrated the reliability of this } \\
\text { system in terms of target } \\
\text { identification. }\end{array}$ & $\begin{array}{l}\text { Since the captured images are } \\
\text { processed, the UAV required more } \\
\text { computational capacity. }\end{array}$ \\
\hline & Ref. [272] & Multiple UAVs/Mobile & Multiple BSs & Disaster area & $\begin{array}{l}\text { Providing a real-time video } \\
\text { monitoring from a UAV to } \\
\text { multiple BSs using a wireless } \\
\text { communication infrastructure }\end{array}$ & $\begin{array}{l}\text { Robust to individual failures of BSs } \\
\text { and also expandable to add more } \\
\text { UAVs. }\end{array}$ & $\begin{array}{l}\text { The communication is lost between } \\
\text { UAVs and BSs when there is } \\
\text { non-LoS. }\end{array}$ \\
\hline & Ref. [273] & Multiple UAVs/Mobile & $\begin{array}{c}\text { multiple } \\
\text { vehicles/single } \\
\text { emergency vehicle }\end{array}$ & Urban area & $\begin{array}{l}\text { Plotting the fastest paths for } \\
\text { emergency vehicles to intervene in } \\
\text { the accident areas. }\end{array}$ & $\begin{array}{l}\text { Significantly minimized the } \\
\text { intervention time of rescue teams. }\end{array}$ & $\begin{array}{l}\text { The coverage of the whole area } \\
\text { requires a high density of UAVs. }\end{array}$ \\
\hline \multirow{4}{*}{ Recovery } & Ref. [275] & Multiple UAVs/Mobile & Multiple BSs & Urban area & $\begin{array}{l}\text { Replacing damaged communication } \\
\text { infrastructures by UAVs }\end{array}$ & $\begin{array}{l}\text { Provided a good network } \\
\text { performance in terms of throughput } \\
\text { and fairness. }\end{array}$ & $\begin{array}{l}\text { The energy consumption is not } \\
\text { well covered in this scheme. }\end{array}$ \\
\hline & Ref. [276] & Multiple UAV-BSs/Mobile & Multiple BSs & Disaster area & $\begin{array}{l}\text { Optimally placing UAV-BSs to } \\
\text { replace damaged infrastructures }\end{array}$ & $\begin{array}{l}\text { Improved the throughput of } \\
\text { coverage. }\end{array}$ & $\begin{array}{l}\text { The trajectories of UAV-BSs are } \\
\text { neglected, which can increase } \\
\text { energy consumption. }\end{array}$ \\
\hline & Ref. [277] & Multiple UAV-BSs/Mobile & Multiple GUs & Disaster area & $\begin{array}{l}\text { Deploying UAV-BSs in emergency } \\
\text { communication scenarios }\end{array}$ & $\begin{array}{l}\text { Improved network throughput } \\
\text { during temporary event. }\end{array}$ & $\begin{array}{l}\text { The altitude, direction, speed, and } \\
\text { the environmental constraints are } \\
\text { not considered during the } \\
\text { deployment of UAV-BSs. }\end{array}$ \\
\hline & Ref. [278] & Multiple UAVs/Mobile & Single BS & Disaster area & $\begin{array}{l}\text { Providing emergency } \\
\text { communication infrastructures } \\
\text { using UAVs }\end{array}$ & $\begin{array}{l}\text { Provided a feasible solution for } \\
\text { emergency communications. }\end{array}$ & $\begin{array}{l}\text { A limited number of UAVs can } \\
\text { increase the energy consumption of } \\
\text { UAVs. }\end{array}$ \\
\hline
\end{tabular}

In recent years, many research endeavors have been conducted on how to use UAVs as a recovery mechanism. More explicitly, in [275], UAVs are exploited to replace, or complement the affected communication infrastructure during a disaster event. Indeed, the flexibility of deployment of UAVs is crucial to serving as aerial BSs improving the coverage of a given area. For this purpose, an optimization problem is illustrated to provide the most appropriate coverage that increases user throughput, while ensuring fairness in the different parts of the affected geographical area. However, this scheme does not cover the energy consumption of UAVs. Similarly, in [276], UAV-BSs aim to explore disaster-affected areas with the objective to be rapidly deployed, efficiently provide coverage, and recover communications. A genetic algorithm is proposed to optimize the appropriate locations of UAV-BSs in order to increase network throughput and to replace damaged infrastructures. Nevertheless, the trajectories of UAV-BSs are omitted, which can enhance the network coverage as well as the energy consumption of UAV-BSs. The same drawback is distinguished in [277], which illustrated that UAVs have the ability to serve as a flying GSM network for providing communication and network coverage to GUs within their ranges. Each UAV is equipped with both a GSM station and a software-defined radio equipment, which are used when there is a failure in the infrastructure of cellular networks. However, the mobility information of UAVs is neglected during the deployment of UAV-BSs. In order to provide a reliable emergency communication solution, Tuna et al. [278] deployed a set of UAVs to establish a recovery communication system for rescue missions after disastrous events. Three subsystems are incorporated in each UAV to perform multi-hop relay communications, formation control, and communications with ground BSs.

Findings: Many recovery strategies are studied in this subsection, and therefore many lessons are learned as follows:

- UAVs acting as aerial BSs play a key role to recover lost communications due to a disaster event and act as a substitution network. Indeed, several optimization problems can be distinguished, such as the energy consumption of UAVs, the optimal trajectories, and the multi-hop relay communications.

- The optimal coverage of a given area needs to be well investigated according to different physical and environmental constraints.

- The flexibility of the proposed architecture to any kinds of situations has to be considered during the conception phase.

\section{Open Research Challenges}

In a disaster situation, UAVs are deployed to monitor areas, to detect victims, and recover communications when existing infrastructures are damaged. However, due to their restricted flying time, UAVs are not able to supervise the disaster area all the time. Moreover, the optimal number of UAVs to perform the disaster management task and the strategy of communication between UAVs are required to be carefully defined according to the surrounding environment and the nature of the mission. As a result, reliable networking models to communicate, self-learning mechanisms to monitor the area, and the initial optimal placement of UAVs, are all in need of more deep studies to overcome the distinguished issues. 


\section{UAV COMPUTING}

Real-time applications and certain latency-sensitive services need intensive computing, thus requiring a high computation capacity, storage, and battery lifetime. With the restricted capacities of UAVs, MEC is a promising technology to handle the aforementioned challenges [279]. Indeed, it exploits the computation capabilities of mobile devices to MEC servers located at the edge of the wireless networks, such as ground BSs with the aim to save energy of mobile devices (e.g., UAVs, IoT devices, etc.) and to provide low-latency services. Another promising technology is Fog Computing, which aims to extend the capacity of the edge devices in order to enhance QoS, to support mobility, and to adapt to the heterogeneity of the network [280]. Finally, another paradigm can be deployed in which collaborative UAVs and their respective resources can be used as mobile cloud computing systems [281]. Indeed, it provides offloading opportunities for users to perform heavy tasks, such as object recognition or augmented reality applications [282]. These three technologies are illustrated in Fig. 21. However, to reliably put on the field these three concepts of computing, several challenges need to be addressed as follows:

- Enhancing the computation offloading at the edge.

- Ensuring a fair allocation of processing load among Fog nodes.

- Maximizing the computational capacity of the whole cloud computing system.

- Minimizing the latency, as well as the processing delays by incorporating efficient mechanisms.

In Table XI, a set of major contributions dedicated to UAV computing is briefly summarized.

\section{A. Edge Computing}

In order to increase the computation capability of UAVs in a cost and energy effective manners, MEC has been adopted to save energy for mobile nodes and to make computation in a low time possible [283]. Since UAVs can offload their intensive computation tasks to ground BSs (i.e., the edge of the network), this helps to increase the application time of UAVs and extend their operation horizon. MEC has been adopted in various contributions that are discussed in the following.
To maintain a sustainable offloading task, Zhou et al. [284] studied UAV-enabled wireless powered mobile edge computing system. This system assumed that energy transmitters and cloudlet are incorporated on UAVs with the aim to transmit energy to several GUs in order to perform task offloading and local computing. Moreover, an energy efficient system has been proposed under the constraints of the size of the input data and the energy recovery model. The two main benefits are distinguished: (i) the computation offloading is enhanced and (ii) the energy consumption is well-regulated between UAVs. Nevertheless, the use of a single UAV to make MEC can be considered as its main drawback. In another use case of UAVs, Kalatzis et al. [285] proposed a UAV-enabled early forest fire detection application based on edge and fog computing principles. This hierarchical architecture, taking advantage of the potent and rich resources of cloud and fog computing, respectively. Moreover, it exploits the sensing abilities of UAVs. All these principles have demonstrated that the adopted Fog computing scheme performed interesting results with the considered scenarios. However, an unfairness allocation is distinguished in the processing load among Fog nodes. In order to deal with the restricted computation resources, offloading computing from UAVs and mobile edge computing in UAVs have been explored in [286] with the aim to enhance the traffic management among UAVs. However, this study considers only a single UAV. Similarly, the research work presented in [287] proposed a mobile edge computing configuration where UAVs are served by their associated ground BSs for an efficient computation offloading. Moreover, this work tries to reduce the completion time of UAVs' mission by optimizing the UAV trajectory, the computation offloading, and the connection to the mobile network. Nevertheless, the energy constraints of UAVs are neglected during the functioning of this scheme. To overcome this restriction, Zhou et al. [288] proposed a UAVenabled mobile edge computing system considering a wireless power transfer. The deployed UAV is composed of both an energy transmitter and a mobile edge computing server to supply user equipment with energy and to provide them mobile edge computing services, respectively. By using alternating algorithms, the computation rate maximization problems are

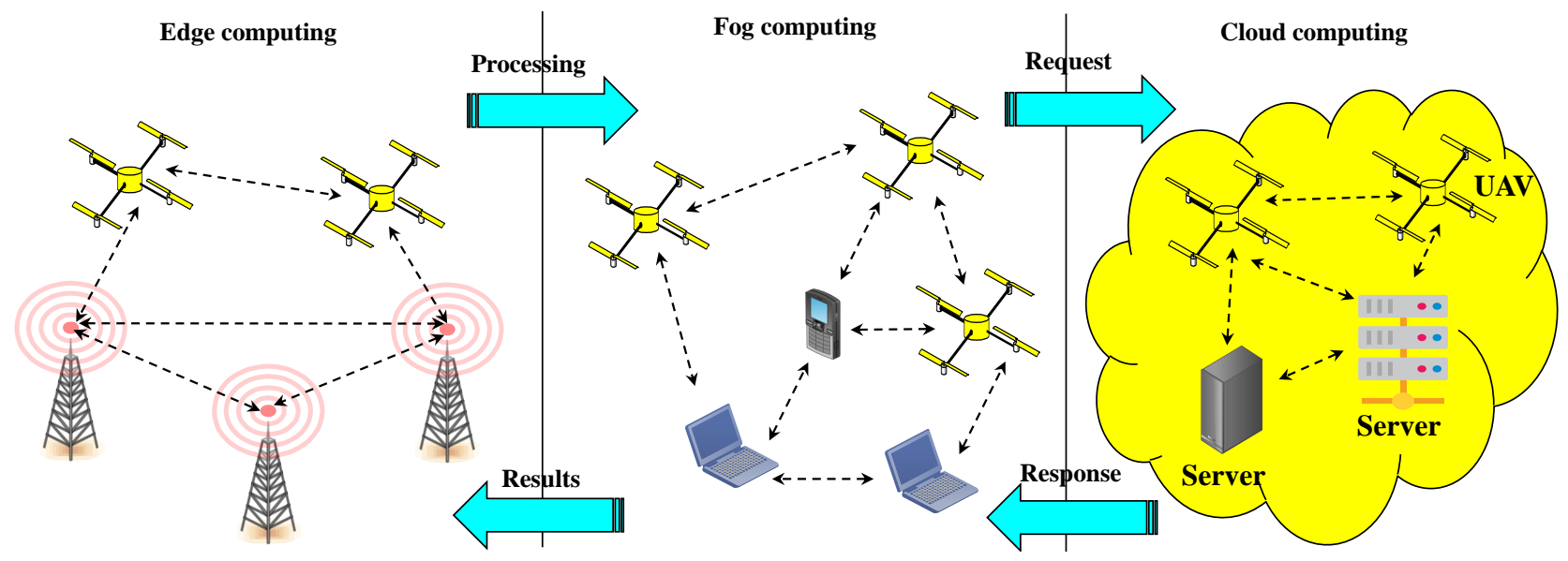

Fig. 21: UAV-assisted computing. 
addressed both by binary and partial computation offloading modes. As a drawback, the operational time of UAVs limits considerably the computational performance.

Findings: As discussed in this subsection, numerous learned lessons can be listed as follows:

- To provide computation offloading opportunities to GUs, UAVs need to have a high processing capacity and an efficient energy consumption strategy in order to act as MEC nodes.

- The operational time and the density of UAVs constitute a crucial challenge to be investigated so that to improve the computational capacity of the whole UAV-assisted system.

- A fairness allocation in the processing load has to be well investigated [289].

\section{B. Cloud and Fog Computing}

Cloud Computing is a promising technique that offers a cost-effective solution for a large amount of data to be processed with a reduced time [290]. Nevertheless, there is a round-trip communication time between the users at the edge and cloud servers along with induced network congestion, which is tolerated by some latency-sensitive applications in certain situations where data processing and decision making are quickly required. To overcome this limitation, Fog Computing leverages computation resources (e.g., mobile computing devices) carried by end users at the edge of the network [291]. This can facilitate processing data of users and provide the feedback in a real-time manner. In the following, a discussion of the major contributions adopting Cloud and Fog Computing paradigms in the context of UAV-assisted networks.

To protect UAVs acting as Fog nodes against GPS spoofing, He et al. [292] proposed two schemes to support the security of UAVs. The first scheme is dedicated to detecting GPS spoofing using both inertial measurement unit (IMU) and monocular camera of the UAV. The second scheme uses an image localization approach in order to support UAV autonomous return using error reduction. As a disadvantage, the centralized nature of this scheme by using both a single BS and one UAV. UAVs acting as Fog nodes can also support some IoT applications to enhance some network performance metrics. For instance, the authors of [293] proposed another work using UAVs as mobile fog nodes to better serve IoT applications in a smart city. This work provides Fog computing capabilities, mobility, flexibility, and dynamically provision features to assist IoT applications in any places and in many scenarios. However, the Fog computing complexity adopted by this work can be considered as its main weaknesses. To avoid the problem of complexity, Chen et al. [294] proposed an intelligent monitoring system in urban areas using the Fog computing paradigm. The latter paradigm is used to get real-time processing in decision making and traffic tracking applications. Moreover, a UAV plays the role of a sensor to monitor, e.g., the vehicles on the roads and the captured images are sent the ground BS. Due to the restricted computing capacity of the ground BS, the intercepted images are firstly sent to Fog computing nodes. As a drawback, the efficiency of this system is completely restricted by using a single UAV acting as a Fog node. Similarly, the work in [295] proposed Fog computing-based monitoring system for transport surveillance. Indeed, this system is able to track vehicles and their speeds in a real-time using single tracking algorithm. However, the monitoring purpose of this system is limited only to one vehicle.

Thus far, many research works have focused on incorporating cloud computing in UAVs to increase network performance in terms of storage and processing. In [296], a mobile cloud computing system based on UAVs is designed. This system investigates the energy-efficient path planning of UAVs and provides computation offloading opportunities to ground mobile stations having restricted processing capabilities. However, mobility and interference among GUs are not supported by this system. A similar work is proposed by Jeong et al. [297]. They adopted a UAV-based moving cloudlet in which UAVs are equipped with a computing processor offload providing computation offloading to ground mobile devices. Indeed, the aim of this work is to minimize the energy consumption of mobile devices by optimizing the bit allocation for uplink/downlink communication in the condition of a predetermined UAV path. As an inconvenience, this neglects the joint local computation and offloading optimization. When the aim of using UAVs is to design cost-effective applications in a timely way, a UAV-cloud platform is proposed in [298] where UAVs can act as servers whose resources (e.g., sensors or smart object) can be exploited by application programming interfaces (APIs). This platform allows users to monitor and request UAV missions. However, there is no consideration of the energy constraints imposed by UAVs. In the case when there is a need to support a large amount of data, the problem of limited resources of UAVs needs to be addressed. Indeed, the authors of [299] proposed a UAV-based cloud system for disaster sensing applications in intermittently connected environments. This system comprises client units hosted on UAVs and a server unit hosted on the remote cloud infrastructure providing service-oriented resource support. Indeed, a set of UAVs is deployed to capture a large amount of video data requiring real-time processing. To improve the performance of this system, data scheduling and processing, video acquisition, and network state measurement, are all considered. In the end, each data is sent to the server and treated by the cloud infrastructure. Nevertheless, the use of a single UAV does not satisfy the monitoring mission requirements.

Findings: Cloud and Fog computing are considered as the hot topics of the moment. Indeed, in this subsection, we have discussed various techniques and schemes and a lot of lessons have been learned, which are summarized as follows:

- UAVs can act as Fog nodes, which can cooperatively accomplish processing tasks, and thus enhance some network performance metrics. However, in many of these contributions, we have distinguished a centralized nature by using a single UAV acting as a Fog node [300].

- UAVs can be used as a cloud platform, making processing and storage tasks with the aim to surpass the restricted capacity of GUs and permanently assist them.

- Several issues are distinguished according to the nature 
TABLE XI: Summary of contributions to UAV computing.

\begin{tabular}{|c|c|c|c|c|c|c|c|}
\hline & & $\begin{array}{l}\text { Density and Mobility of } \\
\text { UAVs }\end{array}$ & $\begin{array}{l}\text { Types of ground } \\
\text { nodes }\end{array}$ & $\begin{array}{l}\text { Deployment } \\
\text { environment }\end{array}$ & Objective & Advantage & Drawback \\
\hline \multirow{5}{*}{ Edge computing } & Ref. [284] & Single UAV/Static & Multiple GUs & Not specified & $\begin{array}{l}\text { Overcoming the limited computation } \\
\text { capacity of GUs by adopting MEC } \\
\text { and wireless power transfer concepts }\end{array}$ & $\begin{array}{l}\text { Enhanced the computation offloading } \\
\text { and optimized the energy consumption } \\
\text { of UAVs. }\end{array}$ & Using a single UAV to perform MEC. \\
\hline & Ref. [285] & Multiple UAVs/Mobile & Single BS & Forest area & $\begin{array}{l}\text { Address the challenges imposed by the } \\
\text { early forest fire detection use case. }\end{array}$ & $\begin{array}{l}\text { The adopted Fog computing principle } \\
\text { demonstrated the most balanced } \\
\text { results among the executed evaluation } \\
\text { scenarios. }\end{array}$ & $\begin{array}{l}\text { The processing load is not fairly } \\
\text { allocated with Fog nodes. }\end{array}$ \\
\hline & Ref. [286] & Single UAV/Mobile & Single BS & Not specified & $\begin{array}{l}\text { Managing UAVs in the uncontrolled } \\
\text { airspace }\end{array}$ & $\begin{array}{l}\text { MEC technology is adopted to } \\
\text { enhance the traffic management of } \\
\text { UAVs. }\end{array}$ & Can be used only with a single UAV. \\
\hline & Ref. [287] & Single UAV/Mobile & Multiple BSs & Not specified & $\begin{array}{l}\text { Offloading computational tasks to } \\
\text { some BSs on the ground }\end{array}$ & $\begin{array}{l}\text { Showed significant performance gain } \\
\text { in terms of mission completion time. }\end{array}$ & $\begin{array}{l}\text { The energy consumption of the UAV } \\
\text { is not considered. }\end{array}$ \\
\hline & Ref. [288] & Single UAV/Static & Multiple IoT devices & Not specified & $\begin{array}{l}\text { Enhancing both the computational } \\
\text { capacity and the operational time of } \\
\text { IoT devices }\end{array}$ & $\begin{array}{l}\text { The allocating resources is } \\
\text { significantly improved. }\end{array}$ & $\begin{array}{l}\text { The computational performance is } \\
\text { restricted by the operational time of } \\
\text { the UAV. }\end{array}$ \\
\hline \multirow{8}{*}{ Cloud and Fog computing } & Ref. [292] & Single UAV/Mobile & Single BS & Airport area & $\begin{array}{l}\text { Detecting GPS spoofing against UAVs } \\
\text { acting as Fog nodes }\end{array}$ & $\begin{array}{l}\text { Demonstrated its effectiveness to } \\
\text { detect GPS spoofing. }\end{array}$ & $\begin{array}{l}\text { Relied on both a centralized } \\
\text { infrastructure and a single UAV. }\end{array}$ \\
\hline & Ref. [293] & Multiple UAVs/Mobile & Multiple sensors/BS & Industrial area & $\begin{array}{l}\text { Supporting some IoT applications by } \\
\text { using Fog computing and UAVs }\end{array}$ & $\begin{array}{l}\text { Provided low latency, efficient } \\
\text { communication, and localization } \\
\text { services. }\end{array}$ & $\begin{array}{l}\text { The architecture of the adopted Fog } \\
\text { principle is complex. }\end{array}$ \\
\hline & Ref. [294] & Single UAV/Mobile & Multiple Fog nodes & Urban area & $\begin{array}{l}\text { Processing real-time monitoring } \\
\text { information using Fog computing }\end{array}$ & $\begin{array}{l}\text { Can handle multiple multiple } \\
\text { monitoring targets without using a } \\
\text { complex algorithm for multi-target } \\
\text { monitoring. }\end{array}$ & $\begin{array}{l}\text { The use of a single UAV limited } \\
\text { considerably the efficiency of this } \\
\text { system. }\end{array}$ \\
\hline & Ref. [295] & Single UAV/Mobile & $\begin{array}{l}\text { Multiple Fog } \\
\text { nodes/BS }\end{array}$ & Urban area & $\begin{array}{l}\text { Urban monitoring solution based on } \\
\text { Fog computing }\end{array}$ & $\begin{array}{l}\text { Detected and tracked a suspicious } \\
\text { vehicle immediately. }\end{array}$ & Tracked only one moving target. \\
\hline & Ref. [296] & Single UAV/Mobile & Multiple GUs & $\begin{array}{c}\text { Infrastructure less } \\
\text { area }\end{array}$ & $\begin{array}{l}\text { Offering computation offloading } \\
\text { opportunities to GUs using a } \\
\text { UAV-based cloud computing }\end{array}$ & $\begin{array}{l}\text { Optimized the bit allocation and the } \\
\text { cloudlet's trajectory to save energy of } \\
\text { GUs. }\end{array}$ & $\begin{array}{l}\text { Did not support multiple moving } \\
\text { interfering GUs. }\end{array}$ \\
\hline & Ref. [297] & Single UAV/Mobile & Single GU & Not specified & $\begin{array}{l}\text { Providing offloading opportunities to } \\
\text { the GU based on a UAV-based cloud } \\
\text { computing }\end{array}$ & $\begin{array}{l}\text { Enhanced the energy consumption of } \\
\text { the GU and its computation } \\
\text { performance. }\end{array}$ & $\begin{array}{l}\text { Neglected both the offloading } \\
\text { optimization and the joint local } \\
\text { computation. }\end{array}$ \\
\hline & Ref. [298] & Multiple UAVs/Mobile & $\begin{array}{l}\text { Multiple ground } \\
\text { nodes }\end{array}$ & Urban area & $\begin{array}{l}\text { Reducing the time and cost to develop } \\
\text { applications using distributed UAVs }\end{array}$ & $\begin{array}{l}\text { Demonstrated high performance with } \\
\text { the broker layer. }\end{array}$ & $\begin{array}{l}\text { Did not consider the energy } \\
\text { constraints of UAVs. }\end{array}$ \\
\hline & Ref. [299] & Single UAV/Mobile & Single BS & Disaster area & $\begin{array}{l}\text { Addressing the problem of limited } \\
\text { resources of UAVs and network using } \\
\text { cloud-supported UAV }\end{array}$ & $\begin{array}{l}\text { Supported a large amount of } \\
\text { intercepted video and processed it in a } \\
\text { real-time. }\end{array}$ & $\begin{array}{l}\text { A single UAV is not always sufficient } \\
\text { to satisfy the monitoring application } \\
\text { requirements. }\end{array}$ \\
\hline
\end{tabular}

of applications in which UAVs are deployed for, such as the cloud and fog computing complexity, the density of UAVs, and the restricted capacity of UAV resources.

\section{Open Research Challenges}

To reduce the latency of many delay-sensitive applications, the computing time needs to be reduced. To do so, several paradigms of computing have emerged, such as mobile edge computing, fog computing, and cloud computing, which provide an important processing and computing capabilities to mobile/static users, thus reducing significantly their energy consumption and the delays of computation. However, many problems arise when the aforementioned paradigms of computing are supported by UAVs, such as their limited energy capacity and their high mobility. This can decrease the computing session continuity for ongoing computing tasks of mobile/static users. This is why researchers have to explore more deeply these challenges and to enhance the functionality of existing approaches.

\section{UAV Secure CommunicAtion}

UAV-assisted systems are exposed to a lot of cyber and physical attacks targeting their communication strategies, their wireless devices, and their movement behavior. In the cyber side, UAV-assisted systems are vulnerable to different vector attacks that are summarized as follows:

- The eavesdropping attacks, which are targeting their wireless communication links between UAVs and between UAVs and ground BSs with the aim to expose private data to attackers [301], [302].

- The jamming attacks directly disable or weakens the communication links within UAV networks. Indeed, the transmission and the reception of messages are disturbed by an opponent transmitting with sufficiently high power on the frequency of the targeted technology [303], [304].

- The false data injection attacks consist to modify the exchanged data, causing several damages, such as taking control of UAVs by deviating them from their paths,

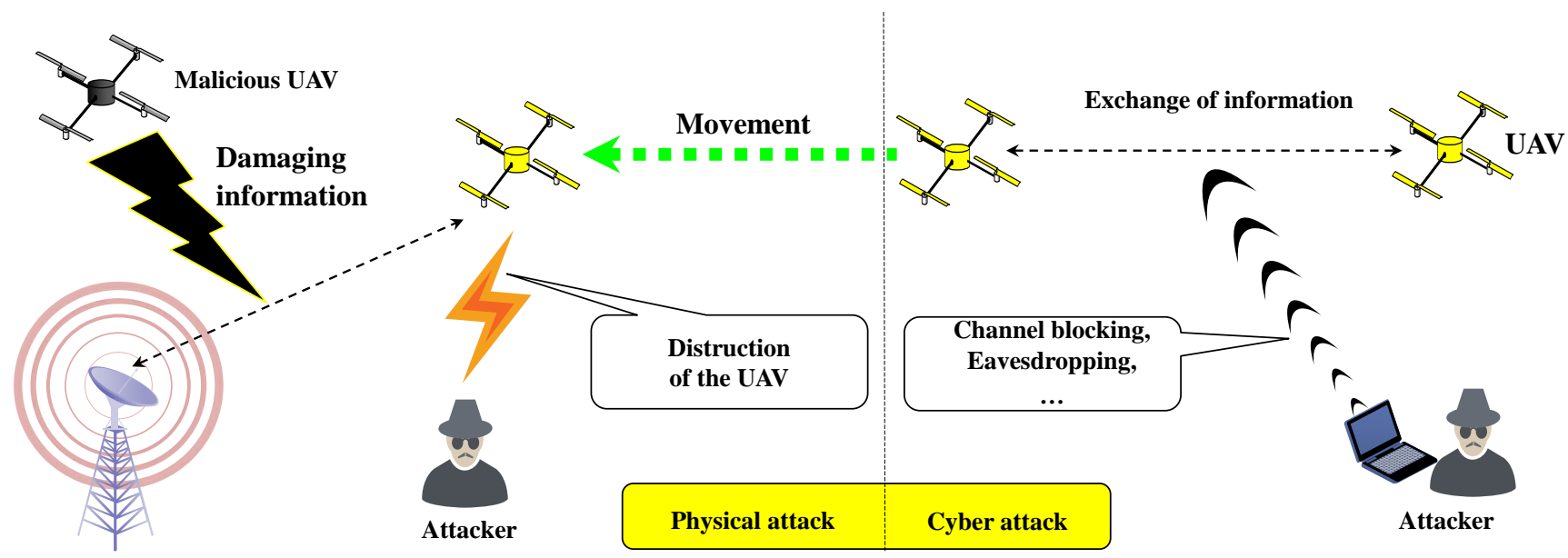

Fig. 22: UAV-assisted secure communication. 
interfering with ground BSs, disseminating false information (e.g., weather conditions, false events, etc.) to their neighbors [305]-[307].

- Recently, various other attacks on UAV networks have also emerged, such as UAV spoofing [308], adversarial machine learning attacks [4], routing attack [309], and grey and black hole attacks [310].

In the physical attacks, UAVs can be pushed to enter in collision with other UAVs by modifying their trajectories and altitudes. Moreover, physical attacks can be in the form of targeting physical channels by generating interference in order to create artificial noise to distort the transmitted messages [311].

To develop efficient and robust security mechanisms, multiple challenges both in the cyber and physical sides should be considered as follows:

- Detecting and countering cyber attacks require the consideration of different UAV constraints, such as the mobility, delivery delays, and energy efficiency.

- Knowing the mechanism of a maximum number of attacks allows to efficiently design the appropriate security scheme.

- Modeling appropriately channel communications permits to better take control of the situation in the case of attacks.

- Avoiding eavesdropping attacks whenever it is possible by proposing efficient physical security schemes.

Fig. 22 illustrates the different use cases of cyber and physical security mechanisms in UAV-assisted networks. Moreover, Table XII summarizes the major contributions handling physical and cyber attacks.

\section{A. Cyber Security}

Different security mechanisms are designed to deal with different cyber attacks. These mechanisms have to guarantee message privacy [312], to prevent external intruders from entering the network [313], and to authenticate nodes [314]. Moreover, the misbehavior of nodes should be monitored using detection techniques. In this subsection, a set of contributions involving UAVs and dealing with cyber attacks is discussed.

To elaborate, Sanjab et al. [315] proposed a zero-sum network interdiction game to address the cyber-physical security threats to UAV communication systems. This scheme considers the scenario of a vendor and an attacker, where the vendor lookup to find the path for UAVs between two different point locations, while the attacker aims to find the optimal attack locations along the path transited by UAVs causing a cyber or physical damage with the aim to increase the travel time of UAVs. As a result, this game can ensure the cyber security of the UAV delivery system. However, due to processing tasks and verification, it increased the delivery time. To be able to detect any malicious anomalies targeting the network, in [316], a hierarchical intrusion detection scheme (IDS) is proposed, which is implemented on both UAVs and ground BSs. The aim of this work is to classify monitoring UAVs and threats to detect malicious activities by combining behavior-based detection and rulebased attack. Therefore, based on these two mechanisms, each
UAV has the ability to act as an observer to hear all traffic in the neighborhood in order to detect any malicious activities. Moreover, a high level of security is demonstrated with a high detection rate. Nevertheless, the functionality of such an approach is restricted only for predefined mobility of UAVs. In another work where the minimum secrecy rate among GUs can be increased, Lee et al. [317] proposed a UAV-assisted secure communication using cooperative jamming, where each UAV communicates with multiple GUs using a time division multiple access (TDMA) protocol to ensure that a transmitted message is intended for one scheduled GU at each time slot. Moreover, by optimizing the UAVs' trajectory, the transmit power of UAVs, user scheduling, and the knowledge of the eavesdropper's location, the minimum secrecy data rate is maximized. In return, this scheme does not support realtime applications. Unlike existing traditional content cache and equal probability content cache strategies, the work in [318] proposed a probabilistic cache strategy to enhance the security performances of heterogeneous networks comprising GUs and UAVs acting as relays. This can effectively enhance the secure cache throughput, but further improvements are required.

Findings: The lessons learned from this subsection can be outlined as follows:

- To detect and counter malicious activities, several cybersecurity techniques have been used. However, they result in numerous other challenges, such as additional delivery delays, the mobility of UAVs, and the energy consumption [319].

- Due to the reduced number of contributions, designing UAV-assisted security strategies is still at the infant stage and requires a lot of research.

- Before a wide deployment of UAVs over our heads, the investigation of all kinds of attacks, as well as the different vulnerabilities of UAV-assisted networks, becomes mandatory.

\section{B. Physical Security}

To overcome physical attacks, such as loss of radio communication [320], misbehavior mobility [321], collisions [322], and others, it is necessary to take security measures in order to ensure the safe hovering of UAVs over a given area. Several contributions are proposed in this side, which are discussed as follows.

In [323], Wang et al. have enhanced significantly the secrecy rate by employing secure mobile relaying systems comprising UAVs in the presence of a ground eavesdropper. It was shown that this work can optimize the transmit power of the relays and mobile devices. In addition, the relaying scheme has proved its efficiency to enhance the secrecy. However, in the case when a single UAV is used, an important delay is introduced. Since there is important information exchanged within the UAV network, any malicious attackers can compromise it. In [324], an authentication algorithm is proposed using an encrypted communication channel in order to protect UAVs from cyber and physical attacks. This promising scheme has the ability to regain control of a compromised UAV using a second channel security system. Though, the physical security 
TABLE XII: Summary of contributions to UAV secure communication.

\begin{tabular}{|c|c|c|c|c|c|c|c|}
\hline & & $\begin{array}{l}\text { Density and Mobility of } \\
\text { UAVs }\end{array}$ & $\begin{array}{l}\text { Types of ground } \\
\text { nodes }\end{array}$ & $\begin{array}{l}\text { Deployment } \\
\text { environment }\end{array}$ & Objective & Advantage & Drawback \\
\hline \multirow{4}{*}{ Cyber security } & Ref. [315] & Single UAV/Mobile & Multiple attackers & Not specified & $\begin{array}{l}\text { Improving the security of UAV } \\
\text { delivery systems }\end{array}$ & $\begin{array}{l}\text { Ensured the security of the UAV } \\
\text { delivery system using various } \\
\text { techniques. }\end{array}$ & $\begin{array}{l}\text { Introduced additional delays in } \\
\text { delivery time. }\end{array}$ \\
\hline & Ref. [316] & Multiple UAVs/Mobile & Multiple BSs & Disaster area & $\begin{array}{l}\text { Detecting malicious anomalies } \\
\text { targeting the network. }\end{array}$ & $\begin{array}{l}\text { Demonstrated a high level of security } \\
\text { with a high detection rate. }\end{array}$ & $\begin{array}{l}\text { Random mobility of UAVs are not } \\
\text { supported. }\end{array}$ \\
\hline & Ref. [317] & Two UAVs/Mobile & Multiple GUs & Not specified & $\begin{array}{l}\text { Increasing the minimum secrecy rate } \\
\text { among GUs }\end{array}$ & $\begin{array}{l}\text { Reduced the secrecy rate among } \\
\text { ground devices. }\end{array}$ & $\begin{array}{l}\text { The delay-constrained applications are } \\
\text { not supported. }\end{array}$ \\
\hline & Ref. [318] & Multiple UAVs/Mobile & Single BS & Not specified & $\begin{array}{l}\text { Ensuring the security of content } \\
\text { transmission }\end{array}$ & Increased the secure cache throughput. & $\begin{array}{l}\text { The transmission reliability and } \\
\text { network security need to be further } \\
\text { improved. }\end{array}$ \\
\hline \multirow{8}{*}{ Physical security } & Ref. [323] & Single UAV/Mobile & Multiple GUs & Urban area & $\begin{array}{l}\text { maximizing the secrecy rate of a } \\
\text { four-node system }\end{array}$ & $\begin{array}{l}\text { Enhanced the secrecy by using an } \\
\text { efficient relaying scheme. }\end{array}$ & $\begin{array}{l}\text { The use of a single UAV can } \\
\text { introduce an additional delay. }\end{array}$ \\
\hline & Ref. [324] & Single UAV/Mobile & Single BS & Not specified & $\begin{array}{l}\text { Maintaining the control of UAV in } \\
\text { case of physical attacks }\end{array}$ & $\begin{array}{l}\text { Designed a second channel security } \\
\text { system to regain the control of the } \\
\text { UAV. }\end{array}$ & $\begin{array}{l}\text { Did not ensure physical security when } \\
\text { a UAV was already compromised. }\end{array}$ \\
\hline & Ref. [325] & Single UAV/Mobile & Single BS & Border area & $\begin{array}{l}\text { Ensuring the safety of the information } \\
\text { transferred by the radio connection } \\
\text { link of the UAV }\end{array}$ & $\begin{array}{l}\text { Detected the vulnerabilities of the } \\
\text { security system in place. }\end{array}$ & $\begin{array}{l}\text { The security equipment of transferring } \\
\text { data is not sufficient. }\end{array}$ \\
\hline & Ref. [327] & Multiple UAVs/Mobile & Single BS & Not specified & $\begin{array}{l}\text { Neglecting spoofing signals by } \\
\text { monitoring the distribution of signal } \\
\text { strength }\end{array}$ & $\begin{array}{l}\text { Surpassed GPS spoofed attacks and } \\
\text { controlled efficiently the UAVs }\end{array}$ & $\begin{array}{l}\text { Did not consider other kinds of } \\
\text { physical attacks. }\end{array}$ \\
\hline & Ref. [326] & Multiple UAVs/Mobile & Single BS & Not specified & $\begin{array}{l}\text { Enhancing the reliability and security } \\
\text { of wireless communications using a } \\
\text { cognitive radio technology }\end{array}$ & $\begin{array}{l}\text { Effectively detected lost links and } \\
\text { jamming. }\end{array}$ & $\begin{array}{l}\text { The energy constraint is omitted in the } \\
\text { conception of this system. }\end{array}$ \\
\hline & Ref. [328] & Multiple UAVs/Mobile & Single BS & Urban/Disaster areas & $\begin{array}{l}\text { Overcoming the problem of } \\
\text { eavesdropping by exploiting physical } \\
\text { layer security mechanisms. }\end{array}$ & $\begin{array}{l}\text { Successfully alter the signal received } \\
\text { from the eavesdroppers. }\end{array}$ & $\begin{array}{l}\text { The robustness of connectivity } \\
\text { between UAVs are neglected. }\end{array}$ \\
\hline & Ref. [329] & Single UAV/Mobile & Two GUs & Urban area & $\begin{array}{l}\text { Using a friendly jammer to assist } \\
\text { secure communications between a } \\
\text { couple of communicating GUs in the } \\
\text { presence of eavesdroppers. }\end{array}$ & $\begin{array}{l}\text { The secrecy performance of the } \\
\text { network is significantly enhanced. }\end{array}$ & $\begin{array}{l}\text { The energy efficiency is not } \\
\text { considered in this scheme. }\end{array}$ \\
\hline & Ref. [330] & Multiple UAVs/Mobile & Single BS/Single GU & Not specified & $\begin{array}{l}\text { Ensuring the security of mmWave } \\
\text { communications against multiple } \\
\text { eavesdroppers by using UAV relays } \\
\text { and jammers. }\end{array}$ & $\begin{array}{l}\text { The secrecy outage probability is } \\
\text { significantly enhanced. }\end{array}$ & $\begin{array}{l}\text { The power allocation between the } \\
\text { different kinds of UAVs is omitted. }\end{array}$ \\
\hline
\end{tabular}

is not guaranteed when a given UAV is already stolen or out of service. To ensure the security of exchanging information through the radio connection link of UAVs, Rudinskas et al. [325] conducted a study of threats and security mechanisms of UAV networks. This helps to identify the vulnerabilities of such networks in order that appropriate secure communication solutions can be deployed to counter them. As an inconvenient, it is not sufficient to cover all kinds of vulnerabilities on different sides. Similarly, to enhance the security and reliability of communication links and the exchange of packets in UAV networks, Reyes et al. [326] suggested using a cognitive radio system as a security solution. This can effectively detect lost links and jamming. Nevertheless, any measures are considered against the energy limitations of this system. GPS spoofing attacks are another kind of attacks that can be overcome. For instance, the authors of [327] investigated how GPS spoofing attack can target the GPS coordinates of UAVs. Indeed, this kind of attacks aims to distort the position calculation of a UAV by jamming its communications. To overcome this attack, this work monitors the distribution of signal strength and by ignoring a spoofed signal. However, the specific use case of this approach can be a disadvantageous factor.

Furthermore, the physical layer has also exploited the different characteristics of wireless channels in order to allow target destinations to successfully receive source information and avoid eavesdropping attacks. For instance, the work in [328] introduced physical layer security approaches dedicated to UAV networks with the aim to avoid the information leakage problem due to eavesdropping. Indeed, two application cases of UAVs (i.e., UAV-BS and UAV-UE) are considered in which an artificial noise along with the source signal are injected to significantly alter the received Signal-to-Interference-plusNoise-Ratio (SINR) at the eavesdroppers. However, due to both the high mobility of UAVs and the need to maintain close communication links with GUs, the link connection with the neighboring UAVs is frequently disconnected. Also, to enhance the physical layer security of a legitimate link, the work in [329] focuses on optimizing the UAV deployment and jamming power allocation. Moreover, a friendly UAV jammer is deployed to transmit artificial noise to improve communication security of the UAV network where the location of the ground eavesdropper is unknown. Nevertheless, due to the introduced jamming, the UAV can quickly exhaust its battery, and thus causing a UAV failure that disturbs the whole system. The authors in [330] have investigated the physical layer security of the millimeter-wave (mmWave) communication system in which a source BS transmits signals to a target destination (GU) through predefined UAVrelays and UAV-jammers all embedded with a directional antenna. Moreover, the authors suppose that there are multiple randomly distributed eavesdroppers on the ground in which their channel qualities are negatively impacted by the existing UAV-jammers, and thus significantly enhancing the secrecy the communication. However, the power allocation between jammer and relay are not investigated.

Findings: Three main lessons are learned from the aforementioned discussion:

- Various external threats are distinguished, which have a formidable strength to annihilate the existing UAV network.

- Protecting the network of any external intruders is of major concerns and require a deep investigation.

- A careful investigation of all kinds of physical attacks needs to be carried out before designing any physical security solutions [331].

\section{Open Research Challenges}

The UAV networks are exposed to different malicious attacks due to their dynamic topologies and their open links. The security issues become more serious when UAV networks are deployed to assist terrestrial networks, where they can face two different other kinds of attacks, such as eavesdropping and ground-air physical attacks. Different security mechanisms 
TABLE XIII: Open research challenges of UAV assistance paradigm.

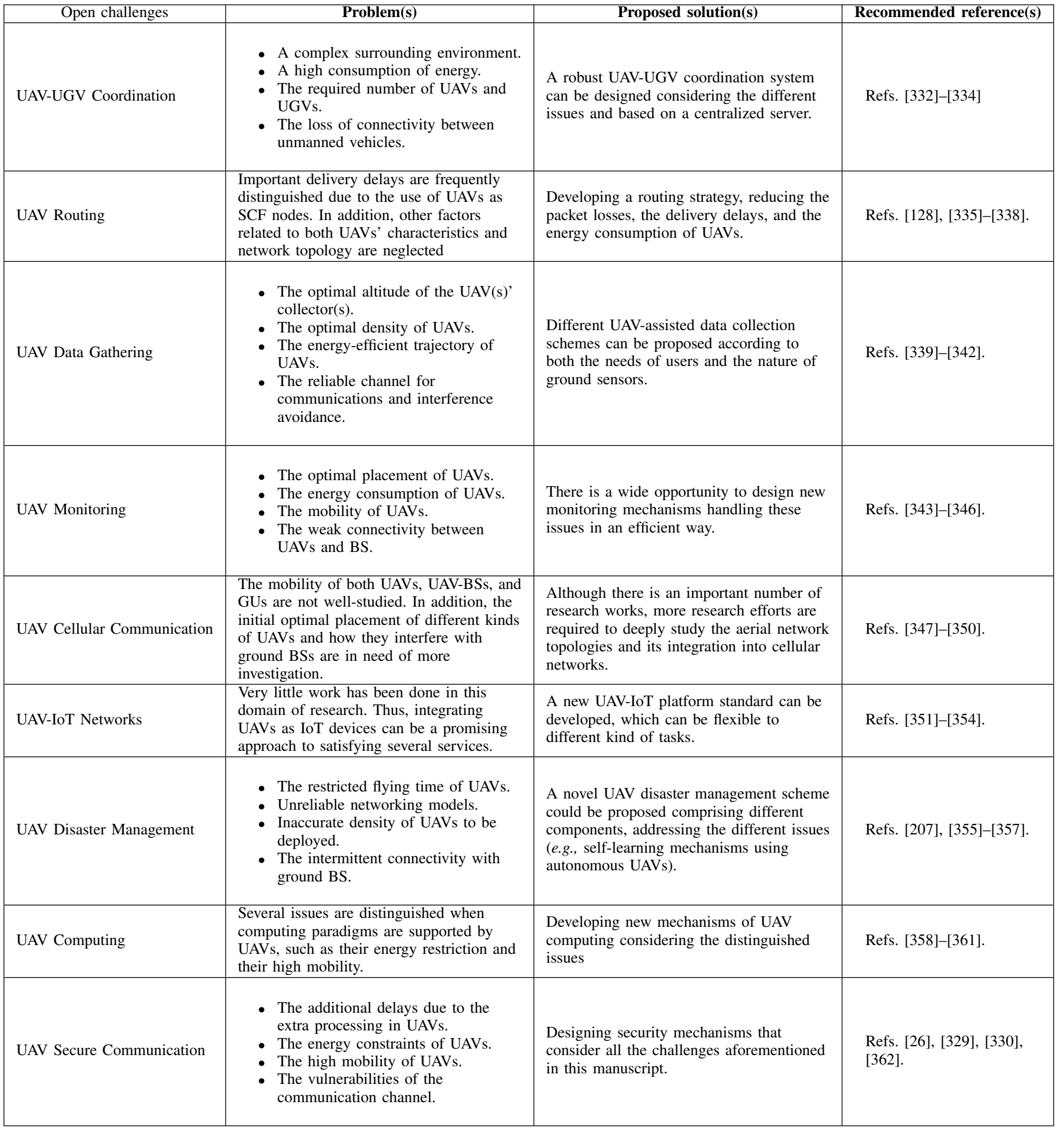

are proposed to deal with both cyber and physical attacks to counteract malicious attacks. However, these proposed security mechanisms provoke other issues, such as the additional delays in delivery time and the energy constraints, which are due to the extra processing carried out in UAVs. Consequently, designing timely security strategies and novel encryption techniques, operating at highly dynamic topologies is of major concerns.

\section{Conclusions and Research Challenges}

The integration of UAVs into the airspace is becoming a reality, leading to the emergence of an important number of UAV-assisted applications. Each application has its own characteristics, features, and competitive advantages. To clarify the difference between these applications, in this comprehensive survey, we have made the following key contributions:

- We have compared the proposed surveys with this survey 
based on different crucial points in order to identify the different novelties of our own survey.

- We have proposed a novel taxonomy which mentioned the majority of use cases of the UAV assistance paradigm.

- We have classified the different UAV assistance paradigms into nine categories that are the most studied in the literature.

- We have investigated the use cases of UAVs as assistants for different purposes.

- For each use case, we have identified the issues, applications, and key open challenges. Moreover, we particularly explored its requirements, its main objective, and its advantage and drawback.

- In each category, we carried out a brief comparative study between the proposals in terms of several parameters.

After describing all these UAV-assisted schemes and techniques along with their learned lessons and open research challenges, it is natural to summarize the main problems that are only addressed ostensibly in the literature. Indeed, there are many challenging issues and shortcomings in each UAVassisted proposal, which are in need of further investigation and most of the time they are left as future works or barely looked at by the authors. For instance, each kind of applications should carefully consider the different constraints related to the use of UAVs and how to overcome them. Also, since UAVs have to be deployed over a given area, the surrounding environment, as well as the wireless interaction with other devices, have to be considered. This allows us to outline future opportunities for researchers who want to embark on a deep study in the UAV assistance paradigm. In this section and for each category, we identify a number of the main open issues, proposed solutions, and recommend some interesting references. In the following, a summary of this discussion is presented in Table XIII.

As a final conclusion, the UAV assistance paradigm is still at the infant stage of research and need more investigation to reach a complete stage of research. As future work, we are currently planning to investigate deeply the concept of visible light communication and its deployment in UAV networks.

\section{ACKNOWLEDGMENT}

This project was funded by the research and development office (RDO) at the ministry of Education, Kingdom of Saudi Arabia, Grant no (HIQI-31-2019). The authors also, acknowledge with thanks research and development office (RDOKAU) at King Abdulaziz University for technical support.

\section{REFERENCES}

[1] "Hank Price Federal Aviation Administration (FAA) Forecast Fiscal Years 2017-2038." https://www.faa.gov/news /fact' sheets/news'story.cfm?newsId=22594, accessed: 2019-04-26.

[2] Z. Yuan, J. Jin, L. Sun, K.-W. Chin, and G.-M. Muntean, "UltraReliable IoT Communications with UAVs: A Swarm Use Case," IEEE Communications Magazine, vol. 56, no. 12, pp. 90-96, 2018.

[3] L. Ferranti, F. Cuomo, S. Colonnese, and T. Melodia, "Drone cellular networks: Enhancing the quality of experience of video streaming applications," Ad Hoc Networks, vol. 78, pp. 1-12, 2018.

[4] U. Challita, A. Ferdowsi, M. Chen, and W. Saad, "Machine Learning for Wireless Connectivity and Security of Cellular-Connected UAVs," IEEE Wireless Communications, vol. 26, no. 1, pp. 28-35, 2019.
[5] B. Hament and P. Oh, "Unmanned aerial and ground vehicle (UAVUGV) system prototype for civil infrastructure missions," in Proceedings of the IEEE International Conference on Consumer Electronics (ICCE). IEEE, 2018, pp. 1-4.

[6] D. Ebrahimi, S. Sharafeddine, P.-H. Ho, and C. Assi, "UAV-Aided Projection-Based Compressive Data Gathering in Wireless Sensor Networks," IEEE Internet of Things Journal, 2018.

[7] S. Sharafeddine and R. Islambouli, "On-demand deployment of multiple aerial base stations for traffic offloading and network recovery," Computer Networks, vol. 156, pp. 52-61, 2019.

[8] G. Zhang, Q. Wu, M. Cui, and R. Zhang, "Securing UAV communications via joint trajectory and power control," IEEE Transactions on Wireless Communications, vol. 18, no. 2, pp. 1376-1389, 2019.

[9] C. H. Liu, Z. Chen, J. Tang, J. Xu, and C. Piao, "Energy-efficient UAV control for effective and fair communication coverage: A deep reinforcement learning approach," IEEE Journal on Selected Areas in Communications, vol. 36, no. 9, pp. 2059-2070, 2018.

[10] H. Shakhatreh, A. H. Sawalmeh, A. Al-Fuqaha, Z. Dou, E. Almaita, I. Khalil, N. S. Othman, A. Khreishah, and M. Guizani, "Unmanned Aerial Vehicles (UAVs): A Survey on Civil Applications and Key Research Challenges," IEEE Access, vol. 7, pp. 48 572-48 634, 2019.

[11] H. Yuan, C. Maple, and K. Ghirardello, "Dynamic Route Selection for Vehicular Store-Carry-Forward Networks and Misbehaviour Vehicles Analysis," in Proceedings of the IEEE 88th Vehicular Technology Conference (VTC-Fall). IEEE, 2019, pp. 1-5.

[12] G. K. Xilouris, M. C. Batistatos, G. E. Athanasiadou, G. Tsoulos, H. B. Pervaiz, and C. C. Zarakovitis, "UAV-Assisted 5G Network Architecture with Slicing and Virtualization," in Proceedings of the IEEE Globecom Workshops (GC Wkshps). IEEE, 2018, pp. 1-7.

[13] K. Li, R. C. Voicu, S. S. Kanhere, W. Ni, and E. Tovar, "Energy Efficient Legitimate Wireless Surveillance of UAV Communications," IEEE Transactions on Vehicular Technology, vol. 68, no. 3, pp. 22832293, 2019.

[14] A. P. Cracknell, "UAVs: regulations and law enforcement," International Journal of Remote Sensing, vol. 38, no. 8-10, pp. 3054-3067, 2017.

[15] E. Vattapparamban, İ. Güvenç, A. İ. Yurekli, K. Akkaya, and S. Uluağaç, "Drones for smart cities: Issues in cybersecurity, privacy, and public safety," in Proceedings of the International Wireless Communications and Mobile Computing Conference (IWCMC). IEEE, 2016, pp. 216-221.

[16] G. Zhang, Q. Wu, M. Cui, and R. Zhang, "Securing UAV communications via trajectory optimization," in Proceedings of the IEEE Global Communications Conference (GLOBECOM'17). IEEE, 2017, pp. 1-6.

[17] A. Fotouhi, H. Qiang, M. Ding, M. Hassan, L. G. Giordano, A. GarciaRodriguez, and J. Yuan, "Survey on UAV cellular communications: Practical aspects, standardization advancements, regulation, and security challenges," IEEE Communications Surveys \& Tutorials, 2019.

[18] M. Mozaffari, W. Saad, M. Bennis, Y.-H. Nam, and M. Debbah, "A tutorial on UAVs for wireless networks: Applications, challenges, and open problems," IEEE Communications Surveys \& Tutorials, 2019.

[19] Y. Zhi, Z. Fu, X. Sun, and J. Yu, "Security and Privacy Issues of UAV: A Survey," Mobile Networks and Applications, pp. 1-7, 2019.

[20] W. Shi, H. Zhou, J. Li, W. Xu, N. Zhang, and X. Shen, "Drone assisted vehicular networks: Architecture, challenges and opportunities," IEEE Network, vol. 32, no. 3, pp. 130-137, 2018.

[21] A. A. Khuwaja, Y. Chen, N. Zhao, M.-S. Alouini, and P. Dobbins, "A survey of channel modeling for UAV communications," IEEE Communications Surveys \& Tutorials, vol. 20, no. 4, pp. 2804-2821, 2018.

[22] M. Campion, P. Ranganathan, and S. Faruque, "A Review and Future Directions of UAV Swarm Communication Architectures," in Proceedings of the IEEE International Conference on Electro/Information Technology (EIT). IEEE, 2018, pp. 0903-0908.

[23] S. Zakaria, M. R. Mahadi, A. F. Abdullah, and K. Abdan, "Aerial Platform Reliability for Flood Monitoring Under Various Weather Conditions: A Review," in Proceedings of the GeoInformation for Disaster Management Conference. Springer, 2018, pp. 295-314.

[24] M. Campion, P. Ranganathan, and S. Faruque, "UAV Swarm Communication and Control Architectures: A Review," Journal of Unmanned Vehicle Systems, no. ja, 2018.

[25] H. Huang and A. Savkin, "Towards the Internet of Flying Robots: A Survey," Sensors, vol. 18, no. 11, p. 4038, 2018.

[26] B. Li, Z. Fei, and Y. Zhang, "UAV communications for $5 \mathrm{G}$ and beyond: Recent advances and future trends," IEEE Internet of Things Journal, 2018 . 
[27] I. Jawhar, N. Mohamed, J. Al-Jaroodi, D. P. Agrawal, and S. Zhang, "Communication and networking of UAV-based systems: Classification and associated architectures," Journal of Network and Computer Applications, vol. 84, pp. 93-108, 2017.

[28] J. Wang, C. Jiang, Z. Han, Y. Ren, R. G. Maunder, and L. Hanzo, "Taking drones to the next level: Cooperative distributed unmannedaerial-vehicular networks for small and mini drones," IEEE vehIcular technology magazIne, vol. 12, no. 3, pp. 73-82, 2017.

[29] C. L. Krishna and R. R. Murphy, "A review on cybersecurity vulnerabilities for Unmanned Aerial Vehicles," in Proceedings of the IEEE International Symposium on Safety, Security and Rescue Robotics (SSRR). IEEE, 2017, pp. 194-199.

[30] C. Stöcker, R. Bennett, F. Nex, M. Gerke, and J. Zevenbergen, "Review of the current state of UAV regulations," Remote sensing, vol. 9, no. 5, p. $459,2017$.

[31] H. González-Jorge, J. Martínez-Sánchez, M. Bueno et al., "Unmanned aerial systems for civil applications: A review," Drones, vol. 1, no. 1, p. 2, 2017.

[32] M. Erdelj and E. Natalizio, "UAV-assisted disaster management: Applications and open issues," in Proceedings of the international conference on computing, networking and communications (ICNC). IEEE, 2016, pp. $1-5$.

[33] L. Gupta, R. Jain, and G. Vaszkun, "Survey of important issues in UAV communication networks," IEEE Communications Surveys \& Tutorials, vol. 18, no. 2, pp. 1123-1152, 2016.

[34] S. Hayat, E. Yanmaz, and R. Muzaffar, "Survey on unmanned aerial vehicle networks for civil applications: A communications viewpoint," IEEE Communications Surveys \& Tutorials, vol. 18, no. 4, pp. 2624 2661, 2016.

[35] K. Kanistras, G. Martins, M. J. Rutherford, and K. P. Valavanis, "Survey of unmanned aerial vehicles (UAVs) for traffic monitoring," Handbook of unmanned aerial vehicles, pp. 2643-2666, 2015.

[36] Y. Saleem, M. H. Rehmani, and S. Zeadally, "Integration of cognitive radio technology with unmanned aerial vehicles: issues, opportunities, and future research challenges," Journal of Network and Computer Applications, vol. 50, pp. 15-31, 2015.

[37] Q. Zhang, M. Mozaffari, W. Saad, M. Bennis, and M. Debbah, "Machine learning for predictive on-demand deployment of UAVs for wireless communications," in Proceedings of the IEEE Global Communications Conference (GLOBECOM). IEEE, 2018, pp. 1-6.

[38] M. Cui, G. Zhang, Q. Wu, and D. W. K. Ng, "Robust trajectory and transmit power design for secure UAV communications," IEEE Transactions on Vehicular Technology, vol. 67, no. 9, pp. 9042-9046, 2018.

[39] D. Yang, Q. Wu, Y. Zeng, and R. Zhang, "Energy tradeoff in groundto-UAV communication via trajectory design," IEEE Transactions on Vehicular Technology, vol. 67, no. 7, pp. 6721-6726, 2018.

[40] W. Mei and R. Zhang, "Uplink cooperative NOMA for cellularconnected UAV," IEEE Journal of Selected Topics in Signal Processing, 2019.

[41] I. Bor-Yaliniz, S. S. Szyszkowicz, and H. Yanikomeroglu, "Environment-aware drone-base-station placements in modern metropolitans," IEEE Wireless Communications Letters, vol. 7, no. 3, pp. 372-375, 2018.

[42] A. Al-Hourani, S. Kandeepan, and A. Jamalipour, "Modeling air-toground path loss for low altitude platforms in urban environments," in Proceedings of the IEEE global communications conference. IEEE, 2014, pp. 2898-2904.

[43] J. Liu, Y. Shi, Z. M. Fadlullah, and N. Kato, "Space-air-ground integrated network: A survey," IEEE Communications Surveys \& Tutorials, vol. 20, no. 4, pp. 2714-2741, 2018.

[44] J. Zhao, F. Gao, Q. Wu, S. Jin, Y. Wu, and W. Jia, "Beam tracking for UAV mounted SatCom on-the-move with massive antenna array," IEEE Journal on Selected Areas in Communications, vol. 36, no. 2, pp. 363-375, 2018.

[45] J. Zhao, F. Gao, G. Ding, T. Zhang, W. Jia, and A. Nallanathan, "Integrating communications and control for UAV systems: Opportunities and challenges," IEEE Access, vol. 6, pp. 67 519-67 527, 2018.

[46] M. Alzenad, A. El-Keyi, and H. Yanikomeroglu, "3-D placement of an unmanned aerial vehicle base station for maximum coverage of users with different QoS requirements," IEEE Wireless Communications Letters, vol. 7, no. 1, pp. 38-41, 2018.

[47] M. M. Azari, F. Rosas, K.-C. Chen, and S. Pollin, "Ultra reliable UAV communication using altitude and cooperation diversity," IEEE Transactions on Communications, vol. 66, no. 1, pp. 330-344, 2018.
[48] M. Liu, J. Yang, and G. Gui, "DSF-NOMA: UAV-Assisted Emergency Communication Technology in a Heterogeneous Internet of Things," IEEE Internet of Things Journal, 2019.

[49] J. Holis and P. Pechac, "Elevation dependent shadowing model for mobile communications via high altitude platforms in built-up areas," IEEE Transactions on Antennas and Propagation, vol. 56, no. 4, pp. 1078-1084, 2008.

[50] P. A. Catherwood, B. Black, E. B. Mohamed, A. A. Cheema, J. Rafferty, and J. A. Mclaughlin, "Radio Channel Characterization of Mid-Band 5G Service Delivery for Ultra-Low Altitude Aerial Base Stations," IEEE Access, vol. 7, pp. 8283-8299.

[51] X. Cai, J. Rodríguez-Piñeiro, X. Yin, N. Wang, B. Ai, G. F. Pedersen, and A. P. Yuste, "An empirical air-to-ground channel model based on passive measurements in LTE," IEEE Transactions on Vehicular Technology, vol. 68, no. 2, pp. 1140-1154, 2018.

[52] Q. Lei and M. Rice, "Multipath channel model for over-water aeronautical telemetry," IEEE Transactions on Aerospace and Electronic Systems, vol. 45, no. 2, pp. 735-742, 2009.

[53] Y. S. Meng and Y. H. Lee, "Measurements and characterizations of air-to-ground channel over sea surface at C-band with low airborne altitudes," IEEE Transactions on Vehicular Technology, vol. 60, no. 4 , pp. 1943-1948, 2011.

[54] F. Ono, T. Kagawa, H. Tsuji, R. Miura, and F. Kojima, "Measurements on C-band air-to-air channel for coexistence among multiple unmanned aircraft systems," in Proceedings of the International Conference on Unmanned Aircraft Systems (ICUAS). IEEE, 2017, pp. 1160-1164.

[55] K. Takizawa, F. Ono, M. Suzuki, H. Tsuji, and R. Miura, "Measurement on S-band radio propagation characteristics for unmanned aircraft systems," in Proceedings of the 8th European Conference on Antennas and Propagation (EuCAP 2014). IEEE, 2014, pp. 3068-3072.

[56] C. Loo, "A statistical model for a land mobile satellite link," IEEE transactions on vehicular technology, vol. 34, no. 3, pp. 122-127, 1985.

[57] S.-H. Hwang, K.-J. Kim, J.-Y. Ahn, and K.-C. Whang, "A channel model for nongeostationary orbiting satellite system," in Proceedings of the IEEE 47th Vehicular Technology Conference. Technology in Motion, vol. 1, pp. 41-45.

[58] M. Vázquez-Castro, F. Pérez-Fontán, and B. Arbesser-Rastburg, "Channel modeling for satellite and HAPS system design," Wireless Communications and mobile computing, vol. 2, no. 3, pp. 285-300, 2002.

[59] C. E. Palazzi, C. Roseti, M. Luglio, M. Gerla, M. Sanadidi, and J. Stepanek, "Satellite coverage in urban areas using Unmanned Airborne Vehicles (UAVs)," in Proceedings of the IEEE 59th Vehicular Technology Conference. VTC 2004-Spring (IEEE Cat. No. 04CH37514), vol. 5. IEEE, 2004, pp. 2886-2890.

[60] S. Sekander, H. Tabassum, and E. Hossain, "Multi-tier drone architecture for 5G/B5G cellular networks: Challenges, trends, and prospects," IEEE Communications Magazine, vol. 56, no. 3, pp. 96-103, 2018.

[61] Y. Zeng, R. Zhang, and T. J. Lim, "Wireless communications with unmanned aerial vehicles: Opportunities and challenges," IEEE Communications Magazine, vol. 54, no. 5, pp. 36-42, 2016.

[62] T. Ding, M. Ding, G. Mao, Z. Lin, D. López-Pérez, and A. Y. Zomaya, "Uplink performance analysis of dense cellular networks with LoS and NLoS transmissions," IEEE Transactions on Wireless Communications, vol. 16, no. 4, pp. 2601-2613, 2017.

[63] M. Ding and D. López-Pérez, "Performance impact of base station antenna heights in dense cellular networks," IEEE Transactions on Wireless Communications, vol. 16, no. 12, pp. 8147-8161, 2017.

[64] X. Cao, P. Yang, M. Alzenad, X. Xi, D. Wu, and H. Yanikomeroglu, "Airborne communication networks: A survey," IEEE Journal on Selected Areas in Communications, vol. 36, no. 9, pp. 1907-1926, 2018.

[65] T. Long, M. Ozger, O. Cetinkaya, and O. B. Akan, "Energy neutral internet of drones," IEEE Communications Magazine, vol. 56, no. 1, pp. 22-28, 2018

[66] H. Wang, G. Ding, F. Gao, J. Chen, J. Wang, and L. Wang, "Power control in UAV-supported ultra dense networks: Communications, caching, and energy transfer," IEEE Communications Magazine, vol. 56, no. 6, pp. 28-34, 2018.

[67] A. Richards and J. P. How, "Aircraft trajectory planning with collision avoidance using mixed integer linear programming," in Proceedings of the American Control Conference (ACC'2000), vol. 3. IEEE, 2002, pp. 1936-1941.

[68] C. S. Ma and R. H. Miller, "MILP optimal path planning for realtime applications," in Proceedings of the American Control Conference. IEEE, 2006, pp. 6-pp.

[69] C. Di Franco and G. Buttazzo, "Energy-aware coverage path planning of UAVs," in Proceedings of the IEEE International Conference on 
Autonomous Robot Systems and Competitions. IEEE, 2015, pp. 111117.

[70] E. I. Grøtli and T. A. Johansen, "Path planning for UAVs under communication constraints using SPLAT! and MILP," Journal of Intelligent \& Robotic Systems, vol. 65, no. 1-4, pp. 265-282, 2012.

[71] D. Halperin, B. Greenstein, A. Sheth, and D. Wetherall, "Demystifying 802.11n power consumption," in Proceedings of the international conference on Power aware computing and systems, 2010, p. 1.

[72] R. Purta, S. Nagrecha, and G. Madey, "Multi-hop Communications in a Swarm of UAVs," in Proceedings of the Agent-Directed Simulation Symposium. Society for Computer Simulation International, 2013, p. 5.

[73] M. N. Bashir and K. M. Yusof, "Green Mesh Network of UAVs: A Survey of Energy Efficient Protocols across Physical, Data Link and Network Layers," in Proceedings of the 4th MEC International Conference on Big Data and Smart City (ICBDSC). IEEE, 2019, pp. $1-6$.

[74] O. S. Oubbati, M. Mozaffari, N. Chaib, P. Lorenz, M. Atiquzzaman, and A. Jamalipour, "Ecad: Energy-efficient routing in flying ad hoc networks," International Journal of Communication Systems, vol. 32, no. 18 , p. e4156, 2019.

[75] A. I. Alshabtat, L. Dong, J. Li, and F. Yang, "Low latency routing algorithm for unmanned aerial vehicles ad-hoc networks," International Journal of Electrical and Computer Engineering, vol. 6, no. 1, pp. 48$54,2010$.

[76] Q. Fan, J. Fan, J. Li, and X. Wang, "A multi-hop energy-efficient sleeping MAC protocol based on TDMA scheduling for wireless mesh sensor networks," Journal of Networks, vol. 7, no. 9, p. 1355, 2012.

[77] X. Yang and N. H. Vaidya, "A wakeup scheme for sensor networks: Achieving balance between energy saving and end-to-end delay," in Proceedings of the 10th IEEE Real-Time and Embedded Technology and Applications Symposium (RTAS 2004). IEEE, 2004, pp. 19-26.

[78] P. Rajendran, K. W. Lim, and K. T. Ong, "Power management strategy by enhancing the mission profile configuration of solar-powered aircraft," International Journal of Aerospace Engineering, vol. 2016, 2016.

[79] M. Mozaffari, W. Saad, M. Bennis, and M. Debbah, "Wireless communication using unmanned aerial vehicles (UAVs): Optimal transport theory for hover time optimization," IEEE Transactions on Wireless Communications, vol. 16, no. 12, pp. 8052-8066, 2017.

[80] J. Xu, Y. Zeng, and R. Zhang, "UAV-enabled wireless power transfer: Trajectory design and energy optimization," IEEE Transactions on Wireless Communications, vol. 17, no. 8, pp. 5092-5106, 2018.

[81] Y. Wu, L. Qiu, and J. Xu, "UAV-enabled wireless power transfer with directional antenna: A two-user case," in Proceedings of the 15th International Symposium on Wireless Communication Systems (ISWCS). IEEE, 2018, pp. 1-6.

[82] S. Yin, Y. Zhao, and L. Li, "UAV-assisted cooperative communications with time-sharing SWIPT," in Proceedings of the IEEE International Conference on Communications (ICC). IEEE, 2018, pp. 1-6.

[83] N. Cheng, W. Xu, W. Shi, Y. Zhou, N. Lu, H. Zhou, and X. Shen, "Airground integrated mobile edge networks: Architecture, challenges, and opportunities," IEEE Communications Magazine, vol. 56, no. 8, pp. 26-32, 2018.

[84] Y. Zeng, J. Lyu, and R. Zhang, "Cellular-Connected UAV: Potential, Challenges, and Promising Technologies," IEEE Wireless Communications, vol. 26, no. 1, pp. 120-127, 2019.

[85] R. Verdone and S. Mignardi, "Joint Aerial-Terrestrial Resource Management in UAV-Aided Mobile Radio Networks," IEEE Network, vol. 32, no. 5, pp. 70-75, 2018

[86] M. Monwar, O. Semiari, and W. Saad, "Optimized Path Planning for Inspection by Unmanned Aerial Vehicles Swarm with Energy Constraints," in Proceedings of the IEEE Global Communications Conference (GLOBECOM). IEEE, 2018, pp. 1-6.

[87] T. Baca, D. Hert, G. Loianno, M. Saska, and V. Kumar, "Model predictive trajectory tracking and collision avoidance for reliable outdoor deployment of unmanned aerial vehicles," in Proceedings of the IEEE/RSJ International Conference on Intelligent Robots and Systems (IROS). IEEE, 2018, pp. 6753-6760.

[88] F. Qi, X. Zhu, G. Mang, M. Kadoch, and W. Li, "UAV Network and IoT in the Sky for Future Smart Cities," IEEE Network, vol. 33, no. 2, pp. 96-101, 2019.

[89] L. Liu, S. Zhang, and R. Zhang, "Cooperative Interference Cancellation for Multi-Beam UAV Uplink Communication: A DoF Analysis," in Proceedings of the IEEE Globecom Workshops (GC Wkshps). IEEE, 2018, pp. 1-6.
[90] C. Zhang, W. Zhang, W. Wang, L. Yang, and W. Zhang, "Research Challenges and Opportunities of UAV Millimeter-Wave Communications," IEEE Wireless Communications, vol. 26, no. 1, pp. 58-62, 2019.

[91] S. Bhunia, P. A. Regis, and S. Sengupta, "Distributed adaptive beam nulling to survive against jamming in 3D UAV mesh networks," Computer Networks, vol. 137, pp. 83-97, 2018.

[92] J. Ye, C. Zhang, H. Lei, G. Pan, and Z. Ding, "Secure UAV-to-UAV systems with spatially random UAVs," IEEE Wireless Communications Letters, 2018.

[93] D. A. Hahn, A. Munir, and S. P. Mohanty, "Security and Privacy Issues in Contemporary Consumer Electronics [Energy and Security]," IEEE Consumer Electronics Magazine, vol. 8, no. 1, pp. 95-99, 2019.

[94] V. S. Dwivedi, J. Patrikar, A. Addamane, and A. Ghosh, "MARAAL: A low altitude long endurance solar powered UAV for surveillance and mapping applications," in 2018 23rd International Conference on Methods \& Models in Automation \& Robotics (MMAR). IEEE, 2018 , pp. $449-454$

[95] D. Solomitckii, M. Gapeyenko, V. Semkin, S. Andreev, and Y. Koucheryavy, "Technologies for efficient amateur drone detection in $5 \mathrm{G}$ millimeter-wave cellular infrastructure," IEEE Communications Magazine, vol. 56, no. 1, pp. 43-50, 2018.

[96] H. Shakhatreh, A. Khreishah, and B. Ji, "Providing wireless coverage to high-rise buildings using UAVs," in Proceedings of the IEEE International Conference on Communications (ICC). IEEE, 2017, pp. 1-6.

[97] M. Mozaffari, W. Saad, M. Bennis, and M. Debbah, "Efficient deployment of multiple unmanned aerial vehicles for optimal wireless coverage," IEEE Communications Letters, vol. 20, no. 8, pp. 16471650, 2016.

[98] A. V. Savkin and H. Huang, "A method for optimized deployment of a network of surveillance aerial drones," IEEE Systems Journal, vol. 13, no. 4, pp. 4474-4477, 2019

[99] J. Boubeta-Puig, E. Moguel, F. Sánchez-Figueroa, J. Hernández, and J. C. Preciado, "An autonomous UAV architecture for remote sensing and intelligent decision-making," IEEE Internet Computing, vol. 22, no. 3, pp. 6-15, 2018

[100] S. Sawadsitang, D. Niyato, P.-S. Tan, and P. Wang, "Joint Ground and Aerial Package Delivery Services: A Stochastic Optimization Approach," IEEE Transactions on Intelligent Transportation Systems, vol. 20, no. 6, pp. 2241-2254, 2018.

[101] S. J. Kim, Y. Jeong, S. Park, K. Ryu, and G. Oh, "A survey of drone use for entertainment and AVR (augmented and virtual reality)," in Augmented Reality and Virtual Reality. Springer, 2018, pp. 339-352.

[102] J. Plachy, Z. Becvar, P. Mach, R. Marik, and M. Vondra, "Joint positioning of Flying Base stations and Association of Users: Evolutionarybased approach," IEEE Access, vol. 7, pp. 11454-11 463, 2019.

[103] S. Jayavelu, S. Sundaram et al., "Dynamic area coverage for multi-UAV using distributed UGVs: A two-stage density estimation approach," in Proceedings of the Second IEEE International Conference on Robotic Computing (IRC). IEEE, 2018, pp. 165-166.

[104] A. Aghaeeyan, F. Abdollahi, and H. A. Talebi, "UAV-UGVs cooperation: With a moving center based trajectory," Robotics and Autonomous Systems, vol. 63, pp. 1-9, 2015.

[105] F. Guérin, F. Guinand, J.-F. Brethé, H. Pelvillain, A. Zentout et al., "Vision based target tracking using an Unmanned Aerial Vehicle," in Proceedings of the IEEE International Workshop on Advanced Robotics and its Social Impacts (ARSO). IEEE, 2015, pp. 1-6.

[106] F. Ropero, P. Muñoz, and M. D. R-Moreno, "TERRA: A path planning algorithm for cooperative UGV-UAV exploration," Engineering Applications of Artificial Intelligence, vol. 78, pp. 260-272, 2019.

[107] A. Lakas, B. Belkhouche, O. Benkraouda, A. Shuaib, and H. J. Alasmawi, "A Framework for a Cooperative UAV-UGV System for Path Discovery and Planning," in Proceedings of the International Conference on Innovations in Information Technology (IIT). IEEE, 2018, pp. 42-46.

[108] F. Duchoň, A. Babinec, M. Kajan, P. Beňo, M. Florek, T. Fico, and L. Jurišica, "Path Planning with Modified a Star Algorithm for a Mobile Robot," Procedia Engineering, vol. 96, pp. 59-69, 2014.

[109] B. Arbanas, A. Ivanovic, M. Car, M. Orsag, T. Petrovic, and S. Bogdan, "Decentralized planning and control for UAV-UGV cooperative teams," Autonomous Robots, vol. 42, no. 8, pp. 1601-1618, 2018.

[110] J. Li, G. Deng, C. Luo, Q. Lin, Q. Yan, and Z. Ming, "A hybrid path planning method in unmanned air/ground vehicle (UAV/UGV) cooperative systems," IEEE Transactions on Vehicular Technology, vol. 65 , no. 12, pp. 9585-9596, 2016.

[111] H. Yu, K. Meier, M. Argyle, and R. W. Beard, "Cooperative path planning for target tracking in urban environments using unmanned 
air and ground vehicles," IEEE/ASME Transactions on Mechatronics, vol. 20, no. 2, pp. 541-552, 2015.

[112] L. Feng, C. Wiltsche, L. Humphrey, and U. Topcu, "Controller synthesis for autonomous systems interacting with human operators," in Proceedings of the acm/ieee sixth international conference on cyberphysical systems. ACM, 2015, pp. 70-79.

[113] S. S. Ponda, L. B. Johnson, A. Geramifard, and J. P. How, "Cooperative mission planning for multi-UAV teams," Handbook of Unmanned Aerial Vehicles, pp. 1447-1490, 2015.

[114] P. Tokekar, J. Vander Hook, D. Mulla, and V. Isler, "Sensor planning for a symbiotic UAV and UGV system for precision agriculture," IEEE Transactions on Robotics, vol. 32, no. 6, pp. 1498-1511, 2016.

[115] K. A. Ghamry, M. A. Kamel, and Y. Zhang, "Cooperative forest monitoring and fire detection using a team of UAVs-UGVs," in Proceedings of the International Conference on Unmanned Aircraft Systems (ICUAS). IEEE, 2016, pp. 1206-1211.

[116] L. Cantelli, M. Mangiameli, C. D. Melita, and G. Muscato, "UAV/UGV cooperation for surveying operations in humanitarian demining," in Proceedings of the IEEE international symposium on safety, security, and rescue robotics (SSRR). IEEE, 2013, pp. 1-6.

[117] M. Saska, T. Krajnik, and L. Pfeucil, "Cooperative $\mu$ UAV-UGV autonomous indoor surveillance," in Proceedings of the International Multi-Conference on Systems, Sygnals \& Devices. IEEE, 2012, pp. $1-6$.

[118] C. Phan and H. H. Liu, "A cooperative UAV/UGV platform for wildfire detection and fighting," in Proceedings of the Asia Simulation Conference and the 7th International Conference on System Simulation and Scientific Computing. IEEE, 2008, pp. 494-498.

[119] J. Chen, X. Zhang, B. Xin, and H. Fang, "Coordination between unmanned aerial and ground vehicles: A taxonomy and optimization perspective," IEEE transactions on cybernetics, vol. 46, no. 4, pp. 959972, 2015.

[120] R. Cajo, T. T. Mac, D. Plaza, C. Copot, R. De Keyser, and C. Ionescu, "A Survey on Fractional Order Control Techniques for Unmanned Aerial and Ground Vehicles," IEEE Access, pp. 66 864-66 878, 2019.

[121] S. Minaeian, J. Liu, and Y.-J. Son, "Vision-based target detection and localization via a team of cooperative UAV and UGVs," IEEE Transactions on systems, man, and cybernetics: systems, vol. 46, no. 7 , pp. 1005-1016, 2016.

[122] L. Barnes, R. Garcia, M. Fields, and K. Valavanis, "Swarm formation control utilizing ground and aerial unmanned systems," in Proceedings of the IEEE/RSJ International Conference on Intelligent Robots and Systems. IEEE, 2008, pp. 4205-4205.

[123] A. S. Brandao, J. A. Sarapura, M. d. O. Eliete, M. Sarcinelli-Filho, and R. Carelli, "Decentralized control of a formation involving a miniature helicopter and a team of ground robots based on artificial vision," in Proceedings of the Latin American Robotics Symposium and Intelligent Robotics Meeting. IEEE, 2010, pp. 126-131.

[124] L. Barnes, R. Garcia, M. A. Fields, and K. Valavanis, "Adaptive swarm formation control for hybrid ground and aerial assets," in Proceedings of the Cutting Edge Robotics. IntechOpen, 2010.

[125] H. Wu, X. Tao, N. Zhang, and X. Shen, "Cooperative UAV clusterassisted terrestrial cellular networks for ubiquitous coverage," IEEE Journal on Selected Areas in Communications, vol. 36, no. 9, pp. 2045 2058, 2018.

[126] V. V. Chetlur and H. S. Dhillon, "Downlink coverage analysis for a finite 3-D wireless network of Unmanned Aerial Vehicles," IEEE Transactions on Communications, vol. 65, no. 10, pp. 4543-4558, 2017.

[127] C. Wang, J. Wang, Y. Shen, and X. Zhang, "Autonomous Navigation of UAVs in Large-scale Complex Environments: A Deep Reinforcement Learning Approach," IEEE Transactions on Vehicular Technology, pp. 2124-2136, 2019.

[128] O. S. Oubbati, A. Lakas, F. Zhou, M. Günes, and M. B. Yagoubi, "A survey on position-based routing protocols for Flying Ad hoc Networks (FANETs)," Vehicular Communications, vol. 10, pp. 29-56, 2017.

[129] O. S. Oubbati, A. Lakas, N. Lagraa, and M. B. Yagoubi, "CRUV: Connectivity-based traffic density aware routing using UAVs for VANETs," in Proceedings of the International Conference on Connected Vehicles and Expo (ICCVE). IEEE, 2015, pp. 68-73.

[130] N. Chaib, O. S. Oubbati, M. L. Bensaad, A. Lakas, P. Lorenz, and A. Jamalipour, "BRT: Bus-Based Routing Technique in Urban Vehicular Networks," IEEE Transactions on Intelligent Transportation Systems, 2019

[131] O. S. Oubbati, A. Lakas, M. Güneş, F. Zhou, and M. B. Yagoubi, "UAV-assisted reactive routing for urban VANETs," in Proceedings of the Symposium on Applied Computing, 2017, pp. 651-653.
[132] O. S. Oubbati, N. Chaib, A. Lakas, P. Lorenz, and A. Rachedi, "UAVassisted supporting services connectivity in urban VANETs," IEEE Transactions on Vehicular Technology, vol. 68, no. 4, pp. 3944-3951, 2019.

[133] O. Sami Oubbati, N. Chaib, A. Lakas, S. Bitam, and P. Lorenz, "U2RV: UAV-assisted reactive routing protocol for VANETs," International Journal of Communication Systems, p. e4104.

[134] X. Fan, C. Huang, B. Fu, S. Wen, and X. Chen, "UAV-Assisted Data Dissemination in Delay-Constrained VANETs," Mobile Information Systems, vol. 2018, 2018.

[135] W. Fawaz, "Effect of non-cooperative vehicles on path connectivity in vehicular networks: A theoretical analysis and UAV-based remedy," Vehicular Communications, vol. 11, pp. 12-19, 2018

[136] W. Fawaz, R. Atallah, C. Assi, and M. Khabbaz, "Unmanned aerial vehicles as store-carry-forward nodes for vehicular networks," IEEE Access, vol. 5, pp. 23710-23718, 2017.

[137] O. S. Oubbati, A. Lakas, F. Zhou, M. Güneş, N. Lagraa, and M. B. Yagoubi, "Intelligent UAV-Assisted Routing Protocol for Urban VANETs," Computer communications, vol. 107, pp. 93-111, 2017.

[138] D. W. Matolak and R. Sun, "Unmanned aircraft systems: Air-ground channel characterization for future applications," IEEE Vehicular Technology Magazine, vol. 10, no. 2, pp. 79-85, 2015.

[139] W. Khawaja, O. Ozdemir, and I. Guvenc, "UAV air-to-ground channel characterization for mmWave systems," in Proceedings of the IEEE 86th Vehicular Technology Conference (VTC-Fall). IEEE, 2017, pp. $1-5$.

[140] Y. Zhang, J. Wen, G. Yang, Z. He, and X. Luo, "Air-to-air path loss prediction based on machine learning methods in urban environments," Wireless Communications and Mobile Computing, vol. 2018, 2018.

[141] G. J. Lim, S. Kim, J. Cho, Y. Gong, and A. Khodaei, "Multi-UAV prepositioning and routing for power network damage assessment," IEEE Transactions on Smart Grid, vol. 9, no. 4, pp. 3643-3651, 2016.

[142] I. Jawhar, N. Mohamed, and J. Al-Jaroodi, "UAV-based data communication in wireless sensor networks: Models and strategies," in Proceedings of the International Conference on Unmanned Aircraft Systems (ICUAS). IEEE, 2015, pp. 687-694.

[143] E. P. De Freitas, T. Heimfarth, I. F. Netto, C. E. Lino, C. E. Pereira A. M. Ferreira, F. R. Wagner, and T. Larsson, "UAV relay network to support WSN connectivity," in Proceedings of the International Congress on Ultra Modern Telecommunications and Control Systems. IEEE, 2010, pp. 309-314.

[144] A. T. Erman, L. van Hoesel, P. Havinga, and J. Wu, "Enabling mobility in heterogeneous wireless sensor networks cooperating with UAVs for mission-critical management," IEEE Wireless Communications, vol. 15, no. 6 , pp. $38-46,2008$.

[145] M. Le, J.-S. Park, and M. Gerla, "UAV assisted disruption tolerant routing," in Proceedings of the IEEE Conference on Military Communications (MILCOM), 2006, pp. 1-5.

[146] C. Perkins, E. Belding-Royer, and S. Das, "Ad hoc on-demand distance vector (AODV) routing," Tech. Rep., 2003.

[147] D. L. Gu, G. Pei, H. Ly, M. Gerla, B. Zhang, and X. Hong, "UAV aided intelligent routing for ad-hoc wireless network in single-area theater," in Proceedings of the IEEE Wireless Communications and Networking Conference, vol. 3. IEEE, 2000, pp. 1220-1225.

[148] S. Liu, Z. Wei, Z. Guo, X. Yuan, and Z. Feng, "Performance Analysis of UAVs Assisted Data Collection in Wireless Sensor Network," in Proceedings of the IEEE 87th Vehicular Technology Conference (VTC Spring). IEEE, 2018, pp. 1-5.

[149] B. Liu and H. Zhu, "Energy-Effective Data Gathering for UAV-Aided Wireless Sensor Networks," Sensors, vol. 19, no. 11, p. 2506, 2019.

[150] J. Gong, T.-H. Chang, C. Shen, and X. Chen, "Flight time minimization of UAV for data collection over wireless sensor networks," IEEE Journal on Selected Areas in Communications, vol. 36, no. 9, pp. 19421954, 2018.

[151] C. Zhan, Y. Zeng, and R. Zhang, "Trajectory Design for Distributed Estimation in UAV-Enabled Wireless Sensor Network," IEEE Transactions on Vehicular Technology, vol. 67, no. 10, pp. 10 155-10 159, 2018

[152] C. Liu, J. Lee, and T. Q. Quek, "Safeguarding UAV Communications Against Full-Duplex Active Eavesdropper," IEEE Transactions on Wireless Communications, 2019.

[153] D. Popescu, C. Dragana, F. Stoican, L. Ichim, and G. Stamatescu, "A collaborative UAV-WSN network for monitoring large areas," Sensors, vol. 18, no. 12, p. 4202, 2018.

[154] C. Zhan, Y. Zeng, and R. Zhang, "Energy-efficient data collection in UAV enabled wireless sensor network," IEEE Wireless Communications Letters, vol. 7, no. 3, pp. 328-331, 2018. 
[155] Q. Wu, P. Sun, and A. Boukerche, "An Energy-Efficient UAV-Based Data Aggregation Protocol in Wireless Sensor Networks," in Proceedings of the 8th ACM Symposium on Design and Analysis of Intelligent Vehicular Networks and Applications. ACM, 2018, pp. 34-40.

[156] S. Verma, N. Sood, and A. K. Sharma, "A novelistic approach for energy efficient routing using single and multiple data sinks in heterogeneous wireless sensor network," Peer-to-Peer Networking and Applications, vol. 12, no. 5, pp. 1110-1136, 2019.

[157] S. Verma, N. Sood, and A. K. Sharma, "Design of a novel routing architecture for harsh environment monitoring in heterogeneous WSN," IET Wireless Sensor Systems, vol. 8, no. 6, pp. 284-294, 2018.

[158] C. Y. Tazibt, M. Bekhti, T. Djamah, N. Achir, and K. Boussetta, "Wireless sensor network clustering for UAV-based data gathering," in Proceedings of the Wireless Days. IEEE, 2017, pp. 245-247.

[159] H. Cao, Y. Liu, X. Yue, and W. Zhu, "Cloud-assisted UAV data collection for multiple emerging events in distributed WSNs," Sensors, vol. 17, no. 8, p. 1818, 2017.

[160] S. Say, H. Inata, J. Liu, and S. Shimamoto, "Priority-based data gathering framework in UAV-assisted wireless sensor networks," IEEE Sensors Journal, vol. 16, no. 14, pp. 5785-5794, 2016.

[161] Q. Wu, W. Chen, D. W. K. Ng, and R. Schober, "Spectral and energyefficient wireless powered IoT networks: NOMA or TDMA?" IEEE Transactions on Vehicular Technology, vol. 67, no. 7, pp. 6663-6667, 2018.

[162] O. Elijah, T. A. Rahman, I. Orikumhi, C. Y. Leow, and M. N. Hindia, "An overview of Internet of things (IoT) and data analytics in agriculture: Benefits and challenges," IEEE Internet of Things Journal, vol. 5 , no. 5 , pp. 3758-3773, 2018 .

[163] H. Wang, J. Wang, G. Ding, L. Wang, T. A. Tsiftsis, and P. K. Sharma, "Resource allocation for energy harvesting-powered D2D communication underlaying UAV-assisted networks," IEEE Transactions on Green Communications and Networking, vol. 2, no. 1, pp. 14-24, 2018.

[164] S. Arabi, H. Elbiaze, E. Sabir, and M. Sadik, "Tradeoffs for Data Collection and Wireless Energy Transfer Dilemma in IoT Environments," in Proceedings of the IEEE International Conference on Communications (ICC). IEEE, 2018, pp. 1-6.

[165] S. Arabi, E. Sabir, H. Elbiaze, and M. Sadik, "Data Gathering and Energy Transfer Dilemma in UAV-Assisted Flying Access Network for IoT," Sensors, vol. 18, no. 5, p. 1519, 2018.

[166] Q. Pan, X. Wen, Z. Lu, L. Li, and W. Jing, "Dynamic Speed Control of Unmanned Aerial Vehicles for Data Collection under Internet of Things," Sensors, vol. 18, no. 11, p. 3951, 2018.

[167] S. Goudarzi, N. Kama, M. H. Anisi, S. Zeadally, and S. Mumtaz, "Data collection using unmanned aerial vehicles for Internet of Things platforms," Computers \& Electrical Engineering, vol. 75, pp. 1-15, 2019.

[168] S. K. Datta, J.-L. Dugelay, and C. Bonnet, "IoT Based UAV Platform for Emergency Services," in Proceedings of the International Conference on Information and Communication Technology Convergence (ICTC). IEEE, 2018, pp. 144-147.

[169] K. Van Beeck, T. Tuytelaars, D. Scarramuza, and T. Goedemé, "RealTime Embedded Computer Vision on UAVs," in European Conference on Computer Vision. Springer, 2018, pp. 3-10.

[170] S. Jung, Y. Jo, and Y.-J. Kim, "Aerial Surveillance with Low-Altitude Long-Endurance Tethered Multirotor UAVs Using Photovoltaic Power Management System," Energies, vol. 12, no. 7, p. 1323, 2019.

[171] A. V. Savkin and H. Huang, "Asymptotically Optimal Deployment of Drones for Surveillance and Monitoring," Sensors, vol. 19, no. 9, p. 2068, 2019.

[172] V. Sharma, I. You, G. Pau, M. Collotta, J. Lim, and J. Kim, "Lorawanbased energy-efficient surveillance by drones for intelligent transportation systems," Energies, vol. 11, no. 3, p. 573, 2018.

[173] R. Reshma, T. Ramesh, and P. Sathishkumar, "Security situational aware intelligent road traffic monitoring using UAVs," in Proceedings of the international conference on VLSI systems, architectures, technology and applications (VLSI-SATA). IEEE, 2016, pp. 1-6.

[174] M. Li, L. Zhen, S. Wang, W. Lv, and X. Qu, "Unmanned aerial vehicle scheduling problem for traffic monitoring," Computers \& Industrial Engineering, vol. 122, pp. 15-23, 2018.

[175] J. Y. Chow, "Dynamic UAV-based traffic monitoring under uncertainty as a stochastic arc-inventory routing policy," International Journal of transportation science and technology, vol. 5, no. 3, pp. 167-185, 2016.

[176] R. Ke, Z. Li, J. Tang, Z. Pan, and Y. Wang, "Real-time traffic flow parameter estimation from UAV video based on ensemble classifier and optical flow," IEEE Transactions on Intelligent Transportation Systems, vol. 20 , no. 1 , pp. 54-64, 2018.
[177] M.-H. Lee and S. Yeom, "Detection and Tracking of Multiple Moving Vehicles with a UAV," International Journal of Fuzzy Logic and Intelligent Systems, vol. 18, no. 3, pp. 182-189, 2018

[178] I. Mademlis, V. Mygdalis, N. Nikolaidis, and I. Pitas, "Challenges in autonomous UAV cinematography: An overview," in Proceedings of the IEEE International Conference on Multimedia and Expo (ICME). IEEE, 2018, pp. 1-6.

[179] D. Kim, M. Liu, S. Lee, and V. R. Kamat, "Remote proximity monitoring between mobile construction resources using camera-mounted UAVs," Automation in Construction, vol. 99, pp. 168-182, 2019.

[180] S. Primatesta, G. Guglieri, and A. Rizzo, "A risk-aware path planning strategy for UAVs in urban environments," Journal of Intelligent \& Robotic Systems, pp. 1-15, 2018.

[181] X. Yue, Y. Liu, J. Wang, H. Song, and H. Cao, "Software defined radio and wireless acoustic networking for amateur drone surveillance," IEEE Communications Magazine, vol. 56, no. 4, pp. 90-97, 2018.

[182] H. Kim, L. Mokdad, and J. Ben-Othman, "Designing UAV surveillance frameworks for smart city and extensive ocean with differential perspectives," IEEE Communications Magazine, vol. 56, no. 4, pp. 98 104, 2018.

[183] J. Gu, T. Su, Q. Wang, X. Du, and M. Guizani, "Multiple moving targets surveillance based on a cooperative network for multi-UAV," IEEE Communications Magazine, vol. 56, no. 4, pp. 82-89, 2018.

[184] S. G. Manyam, S. Rasmussen, D. W. Casbeer, K. Kalyanam, and S. Manickam, "Multi-UAV routing for persistent intelligence surveillance \& reconnaissance missions," in Proceedings of the International Conference on Unmanned Aircraft Systems (ICUAS). IEEE, 2017, pp. 573-580.

[185] A. Koubâa and B. Qureshi, "Dronetrack: Cloud-based real-time object tracking using unmanned aerial vehicles over the internet," IEEE Access, vol. 6, pp. 13810-13824, 2018.

[186] H. Liu, J. Zhang, Y. Pan, G. Shuai, X. Zhu, and S. Zhu, "An efficient approach based on UAV orthographic imagery to map paddy with support of field-level canopy height from point cloud data," IEEE Journal of Selected Topics in Applied Earth Observations and Remote Sensing, vol. 11, no. 6, pp. 2034-2046, 2018.

[187] R. I. Bor-Yaliniz, A. El-Keyi, and H. Yanikomeroglu, "Efficient 3 D placement of an aerial base station in next generation cellular networks," in Proceedings of the IEEE international conference on communications (ICC). IEEE, 2016, pp. 1-5.

[188] N. Zhao, X. Pang, Z. Li, Y. Chen, F. Li, Z. Ding, and M.-S. Alouini, "Joint trajectory and precoding optimization for UAV-assisted NOMA networks," IEEE Transactions on Communications, 2019.

[189] J. Lu, S. Wan, X. Chen, and P. Fan, "Energy-Efficient 3D UAVBS Placement versus Mobile Users' Density and Circuit Power," in Proceedings of the IEEE Globecom Workshops (GC Wkshps). IEEE, 2017, pp. 1-6.

[190] M. Gapeyenko, V. Petrov, D. Moltchanov, S. Andreev, N. Himayat, and Y. Koucheryavy, "Flexible and Reliable UAV-Assisted Backhaul Operation in 5G mmWave Cellular Networks," IEEE Journal on Selected Areas in Communications, vol. 36, no. 11, pp. 2486-2496, 2018.

[191] F. Lagum, I. Bor-Yaliniz, and H. Yanikomeroglu, "Strategic densification with UAV-BSs in cellular networks," IEEE Wireless Communications Letters, vol. 7, no. 3, pp. 384-387, 2018.

[192] V. Sharma, K. Srinivasan, H.-C. Chao, K.-L. Hua, and W.-H. Cheng, "Intelligent deployment of UAVs in 5G heterogeneous communication environment for improved coverage," Journal of Network and Computer Applications, vol. 85, pp. 94-105, 2017.

[193] M. Mozaffari, A. T. Z. Kasgari, W. Saad, M. Bennis, and M. Debbah, "Beyond 5G with UAVs: Foundations of a 3D wireless cellular network," IEEE Transactions on Wireless Communications, vol. 18, no. 1, pp. 357-372, 2019.

[194] R. Ghanavi, E. Kalantari, M. Sabbaghian, H. Yanikomeroglu, and A. Yongacoglu, "Efficient 3D aerial base station placement considering users mobility by reinforcement learning," in Proceedings of the IEEE Wireless Communications and Networking Conference (WCNC). IEEE, 2018, pp. 1-6.

[195] E. Kalantari, H. Yanikomeroglu, and A. Yongacoglu, "On the number and 3D placement of drone base stations in wireless cellular networks,' in Proceedings of the IEEE 84th Vehicular Technology Conference (VTC-Fall). IEEE, 2016, pp. 1-6.

[196] Y. Zeng, R. Zhang, and T. J. Lim, "Throughput maximization for UAVenabled mobile relaying systems," IEEE Transactions on Communications, vol. 64, no. 12, pp. 4983-4996, 2016.

[197] S. Zhang, Y. Zeng, and R. Zhang, "Cellular-enabled UAV communication: A connectivity-constrained trajectory optimization perspective,' 
IEEE Transactions on Communications, vol. 67, no. 3, pp. 2580-2604, 2019.

[198] J. Lyu, Y. Zeng, and R. Zhang, "UAV-aided offloading for cellular hotspot," IEEE Transactions on Wireless Communications, vol. 17, no. 6, pp. 3988-4001, 2018

[199] A. Fotouhi, M. Ding, and M. Hassan, "Service on demand: Drone base stations cruising in the cellular network," in Proceedings of the IEEE Globecom Workshops (GC Wkshps). IEEE, 2017, pp. 1-6.

[200] S.-F. Chou, T.-C. Chiu, Y.-J. Yu, and A.-C. Pang, "Mobile small cell deployment for next generation cellular networks," in Proceedings of the IEEE Global Communications Conference. IEEE, 2014, pp. 4852 4857.

[201] A. Fotouhi, M. Ding, and M. Hassan, "Dynamic base station repositioning to improve spectral efficiency of drone small cells," in Proceedings of the IEEE 18th International Symposium on A World of Wireless, Mobile and Multimedia Networks (WoWMoM). IEEE, 2017, pp. 1-9.

[202] W. Yu, H. Xu, J. Nguyen, E. Blasch, A. Hematian, and W. Gao, "Survey of Public Safety Communications: User-Side and NetworkSide Solutions and Future Directions," IEEE Access, vol. 6, pp. 70397 70425,2018

[203] J. Zhang, Y. Zeng, and R. Zhang, "Spectrum and energy efficiency maximization in UAV-enabled mobile relaying," in Proceedings of the IEEE International Conference on Communications (ICC). IEEE, 2017, pp. 1-6.

[204] K. Li, W. Ni, X. Wang, R. P. Liu, S. S. Kanhere, and S. Jha, "Energyefficient cooperative relaying for Unmanned Aerial Vehicles," IEEE Transactions on Mobile Computing, vol. 15, no. 6, pp. 1377-1386, 2016.

[205] A. E. Abdulla, Z. M. Fadlullah, H. Nishiyama, N. Kato, F. Ono, and R. Miura, "Toward fair maximization of energy efficiency in multiple UAS-aided networks: A game-theoretic methodology," IEEE Transactions on Wireless Communications, vol. 14, no. 1, pp. 305-316, 2015.

[206] S. Kandeepan, K. Gomez, L. Reynaud, and T. Rasheed, "Aerialterrestrial communications: terrestrial cooperation and energy-efficient transmissions to aerial base stations," IEEE Transactions on Aerospace and Electronic Systems, vol. 50, no. 4, pp. 2715-2735, 2014.

[207] H. Yang and X. Xie, "Energy-Efficient Joint Scheduling and Resource Management for UAV-Enabled Multicell Networks," IEEE Systems Journal, 2019.

[208] A. Trotta, M. Di Felice, F. Montori, K. R. Chowdhury, and L. Bononi, "Joint coverage, connectivity, and charging strategies for distributed UAV networks," IEEE Transactions on Robotics, vol. 34, no. 4, pp. 883-900, 2018.

[209] S. Chandrasekharan, K. Gomez, A. Al-Hourani, S. Kandeepan, T. Rasheed, L. Goratti, L. Reynaud, D. Grace, I. Bucaille, T. Wirth et al., "Designing and implementing future aerial communication networks," IEEE Communications Magazine, vol. 54, no. 5, pp. 26-34, 2016.

[210] R. Amorim, H. Nguyen, P. Mogensen, I. Z. Kovács, J. Wigard, and T. B. Sørensen, "Radio channel modeling for UAV communication over cellular networks," IEEE Wireless Communications Letters, vol. 6 , no. 4, pp. 514-517, 2017.

[211] M. Helmy and H. Arslan, "Utilization of aerial heterogeneous cellular networks: Signal-to-interference ratio analysis," Journal of Communications and Networks, vol. 20, no. 5, pp. 484-495, 2018

[212] Z. H. E. Tan, A. Madhukumar, R. P. Sirigina, and A. K. Krishna, "Addressing spectrum efficiency through hybrid-duplex UAV communications: Challenges and opportunities," Vehicular Communications, p. 100235,2020

[213] H. C. Nguyen, R. Amorim, J. Wigard, I. Z. Kovács, T. B. Sørensen, and P. E. Mogensen, "How to ensure reliable connectivity for aerial vehicles over cellular networks," IEEE ACCESS, vol. 6, pp. 12304 12317, 2018

[214] X. Lin, V. Yajnanarayana, S. D. Muruganathan, S. Gao, H. Asplund, H.-L. Maattanen, M. Bergstrom, S. Euler, and Y.-P. E. Wang, "The sky is not the limit: LTE for unmanned aerial vehicles," IEEE Communications Magazine, vol. 56, no. 4, pp. 204-210, 2018

[215] L. Zhou, Z. Yang, S. Zhou, and W. Zhang, "Coverage probability analysis of UAV cellular networks in Urban environments," in Proceedings of the IEEE International Conference on Communications Workshops (ICC Workshops). IEEE, 2018, pp. 1-6.

[216] Y. Chen, N. Zhao, Z. Ding, and M.-S. Alouini, "Multiple UAVs as relays: Multi-hop single link versus multiple dual-hop links," IEEE Transactions on Wireless Communications, vol. 17, no. 9, pp. 63486359, 2018.
[217] Y. Huo, X. Dong, T. Lu, W. Xu, and M. Yuen, "Distributed and multilayer UAV networks for next-generation wireless communication and power transfer: A feasibility study," IEEE Internet of Things Journal, vol. 6, no. 4, pp. 7103-7115, 2019.

[218] L. Wang, H. Yang, J. Long, K. Wu, and J. Chen, "Enabling ultra-dense UAV-aided network with overlapped spectrum sharing: Potential and approaches," IEEE Network, vol. 32, no. 5, pp. 85-91, 2018.

[219] N. H. Motlagh, M. Bagaa, and T. Taleb, "Energy and Delay Aware Task Assignment Mechanism for UAV-based IoT Platform," IEEE Internet of Things Journal, 2019.

[220] D. Sikeridis, E. E. Tsiropoulou, M. Devetsikiotis, and S. Papavassiliou, "Wireless powered Public Safety IoT: A UAV-assisted adaptivelearning approach towards energy efficiency," Journal of Network and Computer Applications, vol. 123, pp. 69-79, 2018.

[221] T. Ahn, J. Seok, I. Lee, and J. Han, "Reliable Flying IoT Networks for UAV Disaster Rescue Operations," Mobile Information Systems, vol. 2018, 2018.

[222] X. Liu, Z. Li, N. Zhao, W. Meng, G. Gui, Y. Chen, and F. Adachi, "Transceiver design and multi-hop D2D for UAV IoT coverage in disasters," IEEE Internet of Things Journal, 2018.

[223] M. Aljehani and M. Inoue, "Multi-UAV tracking and scanning systems in M2M communication for disaster response," in Proceedings of the IEEE 5th Global Conference on Consumer Electronics. IEEE, 2016 , pp. 1-2.

[224] K. Katayama, H. Takahashi, S. Yokoyama, K. Gäfvert, and T. Kinoshita, "Evacuation guidance support using cooperative agent-based IoT devices," in Proceedings of the IEEE 6th Global Conference on Consumer Electronics (GCCE). IEEE, 2017, pp. 1-2

[225] T. Li, K. Ota, T. Wang, X. Li, Z. Cai, and A. Liu, "Optimizing the coverage via the UAVs with lower costs for information-centric Internet of Things," IEEE Access, vol. 7, pp. 15 292-15 309, 2019.

[226] H. Menouar, I. Guvenc, K. Akkaya, A. S. Uluagac, A. Kadri, and A. Tuncer, "UAV-enabled intelligent transportation systems for the smart city: Applications and challenges," IEEE Communications Magazine, vol. 55, no. 3, pp. 22-28, 2017.

[227] M. A. Uddin, A. Mansour, D. Le Jeune, and E. H. M. Aggoune, "Agriculture internet of things: AG-IoT," in Proceedings of the 27th International Telecommunication Networks and Applications Conference (ITNAC). IEEE, 2017, pp. 1-6.

[228] N. Kalatzis, G. Routis, I. Roussaki, and S. Papavassiliou, "Enabling data interoperability for federated IoT experimentation infrastructures," in Proceedings of the Global Internet of Things Summit (GIoTS). IEEE, 2018, pp. 1-6.

[229] A. A. Mutlag, M. K. A. Ghani, N. a. Arunkumar, M. A. Mohamed, and O. Mohd, "Enabling technologies for fog computing in healthcare IoT systems," Future Generation Computer Systems, vol. 90, pp. 62-78, 2019

[230] H.-T. Tseng, H.-G. Hwang, W.-Y. Hsu, P.-C. Chou, I. Chang et al. "IoT-based image recognition system for smart home-delivered meal services," Symmetry, vol. 9, no. 7, p. 125, 2017.

[231] Z. Hu, Z. Bai, Y. Yang, Z. Zheng, K. Bian, and L. Song, "UAV Aided Aerial-Ground IoT for Air Quality Sensing in Smart City: Architecture, Technologies, and Implementation," IEEE Network, vol. 33, no. 2, pp. $14-22,2019$

[232] A. Giyenko and Y. Im Cho, "Intelligent UAV in smart cities using IoT," in Proceedings of the 16th International Conference on Control, Automation and Systems (ICCAS). IEEE, 2016, pp. 207-210.

[233] N. H. Motlagh, M. Bagaa, and T. Taleb, "UAV-based IoT platform: A crowd surveillance use case," IEEE Communications Magazine, vol. 55 , no. 2, pp. 128-134, 2017.

[234] W. Feng, J. Wang, Y. Chen, X. Wang, N. Ge, and J. Lu, "UAV-aided MIMO communications for $5 \mathrm{G}$ internet of things," IEEE Internet of Things Journal, 2018.

[235] G. Aiello, F. Hopps, D. Santisi, and M. Venticinque, "The Employment of Unmanned Aerial Vehicles for Analyzing and Mitigating Disaster Risks in Industrial Sites," IEEE Transactions on Engineering Management, 2020.

[236] E. Guerra, Y. Bolea, J. Gamiz, and A. Grau, "Design and Implementation of a Virtual Sensor Network for Smart Waste Water Monitoring," Sensors, vol. 20, no. 2, p. 358, 2020.

[237] P. Radoglou-Grammatikis, P. Sarigiannidis, T. Lagkas, and I. Moscholios, "A compilation of UAV applications for precision agriculture," Computer Networks, vol. 172, p. 107148, 2020.

[238] V. Puri, A. Nayyar, and L. Raja, "Agriculture drones: A modern breakthrough in precision agriculture," Journal of Statistics and Management Systems, vol. 20, no. 4, pp. 507-518, 2017. 
[239] A. Mukherjee, S. Misra, A. Sukrutha, and N. S. Raghuwanshi, "Distributed aerial processing for IoT-based edge UAV swarms in smart farming," Computer Networks, vol. 167, p. 107038, 2020.

[240] D. Vasisht, Z. Kapetanovic, J. Won, X. Jin, R. Chandra, S. Sinha, A. Kapoor, M. Sudarshan, and S. Stratman, "Farmbeats: An IoT platform for data-driven agriculture," in Proceedings of the 14th $\{$ USENIX\} Symposium on Networked Systems Design and Implementation ( $\{N S D I\}$ 17), 2017, pp. 515-529.

[241] A. K. Saha, J. Saha, R. Ray, S. Sircar, S. Dutta, S. P. Chattopadhyay, and H. N. Saha, "IoT-based drone for improvement of crop quality in agricultural field," in Proceedings of the IEEE 8th Annual Computing and Communication Workshop and Conference (CCWC). IEEE, 2018 , pp. 612-615.

[242] H. Li and A. V. Savkin, "Wireless sensor network based navigation of micro flying robots in the industrial Internet of Things," IEEE Transactions on Industrial Informatics, vol. 14, no. 8, pp. 3524-3533, 2018

[243] Z. Zhou, C. Zhang, C. Xu, F. Xiong, Y. Zhang, and T. Umer, "Energyefficient industrial internet of UAVs for power line inspection in smart grid," IEEE Transactions on Industrial Informatics, vol. 14, no. 6, pp. 2705-2714, 2018.

[244] V. Scilimati, A. Petitti, P. Boccadoro, R. Colella, D. Di Paola, A. Milella, and L. A. Grieco, "Industrial Internet of Things at work: a case study analysis in robotic-aided environmental monitoring," IET wireless sensor systems, vol. 7, no. 5, pp. 155-162, 2017.

[245] F. Al-Turjman and S. Alturjman, "5G/IoT-enabled UAVs for multimedia delivery in industry-oriented applications," Multimedia Tools and Applications, pp. 1-22, 2018.

[246] Q. Zhang, M. Jiang, Z. Feng, W. Li, W. Zhang, and M. Pan, "IoT Enabled UAV: Network Architecture and Routing Algorithm," IEEE Internet of Things Journal, vol. 6, no. 2, pp. 3727-3742, 2019.

[247] A. Ismail, B. Bagula, and E. Tuyishimire, "Internet-of-things in motion: A UAV coalition model for remote sensing in smart cities," Sensors, vol. 18 , no. 7, p. 2184, 2018.

[248] J. Chakareski, "Aerial UAV-IoT sensing for ubiquitous immersive communication and virtual human teleportation," in Proceedings of the IEEE Conference on Computer Communications Workshops (INFOCOM WKSHPS). IEEE, 2017, pp. 718-723.

[249] A. S. Gaur, J. Budakoti, C.-H. Lung, and A. Redmond, "IoT-equipped UAV communications with seamless vertical handover," in Proceedings of the IEEE Conference on Dependable and Secure Computing. IEEE, 2017, pp. 459-465.

[250] S.-J. Yoo, J.-h. Park, S.-h. Kim, and A. Shrestha, "Flying path optimization in UAV-assisted IoT sensor networks," ICT Express, vol. 2, no. 3, pp. $140-144,2016$

[251] O. M. Bushnaq, A. Celik, H. ElSawy, M.-S. Alouini, and T. Y. AlNaffouri, "Aerial data aggregation in IoT networks: Hovering \& traveling time dilemma," in Proceedings of the IEEE Global Communications Conference (GLOBECOM). IEEE, 2018, pp. 1-7.

[252] H. Chae, J. Park, H. Song, Y. Kim, and H. Jeong, "The IoT based automate landing system of a drone for the round-the-clock surveillance solution," in Proceedings of the IEEE international conference on advanced intelligent mechatronics (AIM). IEEE, 2015, pp. 1575-1580.

[253] G. Ding, Q. Wu, L. Zhang, Y. Lin, T. A. Tsiftsis, and Y.-D. Yao, "An amateur drone surveillance system based on the cognitive Internet of Things," IEEE Communications Magazine, vol. 56, no. 1, pp. 29-35, 2018.

[254] H. Kim and J. Ben-Othman, "A Collision-free Surveillance System using Smart UAVs in Multi Domain IoT," IEEE Communications Letters, vol. 22, no. 12, pp. 2587-2590, 2018.

[255] M. Kakooei and Y. Baleghi, "Fusion of satellite, aircraft, and UAV data for automatic disaster damage assessment," International journal of remote sensing, vol. 38, no. 8-10, pp. 2511-2534, 2017.

[256] F. Sharevski, "Leveraging Cellular Intemet-of-Things for Resilient and Robust Disaster Management," in Proceedings of the IEEE Global Communications Conference (GLOBECOM). IEEE, 2018, pp. 1-5.

[257] G. Jiang, R. M. Voyles, and J. J. Choi, "Precision fully-actuated UAV for visual and physical inspection of structures for nuclear decommissioning and search and rescue," in Proceedings of the IEEE International Symposium on Safety, Security, and Rescue Robotics (SSRR). IEEE, 2018, pp. 1-7.

[258] L. Zhong, K. Garlichs, S. Yamada, K. Takano, and Y. Ji, "Mission planning for UAV-based opportunistic disaster recovery networks," in Proceedings of the 15th IEEE Annual Consumer Communications \& Networking Conference (CCNC). IEEE, 2018, pp. 1-6.

[259] P. Oettershagen, T. Stastny, T. Hinzmann, K. Rudin, T. Mantel, A. Melzer, B. Wawrzacz, G. Hitz, and R. Siegwart, "Robotic technolo- gies for solar-powered UAVs: Fully autonomous updraft-aware aerial sensing for multiday search-and-rescue missions," Journal of Field Robotics, vol. 35, no. 4, pp. 612-640, 2018.

[260] M. Erdelj, M. Król, and E. Natalizio, "Wireless sensor networks and multi-UAV systems for natural disaster management," Computer Networks, vol. 124, pp. 72-86, 2017

[261] M. Erdelj, E. Natalizio, K. R. Chowdhury, and I. F. Akyildiz, "Help from the sky: Leveraging UAVs for disaster management," IEEE Pervasive Computing, vol. 16, no. 1, pp. 24-32, 2017.

[262] S. Farfaglia, G. Lollino, M. Iaquinta, I. Sale, P. Catella, M. Martino, and S. Chiesa, "The use of UAV to monitor and manage the territory: perspectives from the SMAT project," in Proceedings of the Engineering Geology for Society and Territory-Volume 5. Springer, 2015, pp. 691-695.

[263] M. Asadpour, D. Giustiniano, K. A. Hummel, and S. Egli, "UAV networks in rescue missions," in Proceedings of the 8th ACM international workshop on Wireless network testbeds, experimental evaluation \& characterization. ACM, 2013, pp. 91-92.

[264] E. T. Alotaibi, S. S. Alqefari, and A. Koubaa, "LSAR: Multi-UAV Collaboration for Search and Rescue Missions," IEEE Access, vol. 7 pp. 55 817-55 832, 2019.

[265] W. Ejaz, A. Ahmed, A. Mushtaq, and M. Ibnkahla, "Energy-efficient task scheduling and physiological assessment in disaster management using UAV-assisted networks," Computer Communications, vol. 155, pp. $150-157,2020$.

[266] M.-C. Mah, H.-S. Lim, and A. W.-C. Tan, "Secrecy improvement via joint optimization of UAV relay flight path and transmit power," Vehicular Communications, vol. 23, p. 100217, 2020.

[267] Z. Yu, Y. Gong, S. Gong, and Y. Guo, "Joint Task Offloading and Resource Allocation in UAV-Enabled Mobile Edge Computing," IEEE Internet of Things Journal, 2020.

[268] S. Hayat, E. Yanmaz, T. X. Brown, and C. Bettstetter, "Multi-objective UAV path planning for search and rescue," in Proceedings of the IEEE International Conference on Robotics and Automation (ICRA). IEEE, 2017, pp. 5569-5574.

[269] M. Silvagni, A. Tonoli, E. Zenerino, and M. Chiaberge, "Multipurpose UAV for search and rescue operations in mountain avalanche events," Geomatics, Natural Hazards and Risk, vol. 8, no. 1, pp. 18-33, 2017.

[270] H. Kurdi, J. How, and G. Bautista, "Bio-inspired algorithm for task allocation in multi-UAV search and rescue missions," in Proceedings of the AIAA Guidance, Navigation, and Control Conference, 2016, p. 1377.

[271] J. Sun, B. Li, Y. Jiang, and C.-y. Wen, "A camera-based target detection and positioning UAV system for search and rescue (SAR) purposes," Sensors, vol. 16, no. 11, p. 1778, 2016

[272] J. Scherer, S. Yahyanejad, S. Hayat, E. Yanmaz, T. Andre, A. Khan, V. Vukadinovic, C. Bettstetter, H. Hellwagner, and B. Rinner, "An autonomous multi-UAV system for search and rescue," in Proceedings of the First Workshop on Micro Aerial Vehicle Networks, Systems, and Applications for Civilian Use. ACM, 2015, pp. 33-38.

[273] O. S. Oubbati, A. Lakas, P. Lorenz, M. Atiquzzaman, and A. Jamalipour, "Leveraging Communicating UAVs for Emergency Vehicle Guidance in Urban Areas," IEEE Transactions on Emerging Topics in Computing, 2019.

[274] A. M. Hayajneh, S. A. R. Zaidi, D. C. McLernon, and M. Ghogho, "Performance analysis of UAV enabled disaster recovery network: A stochastic geometric framework based on matern cluster processes," 2017.

[275] F. Malandrino, C.-F. Chiasserini, C. Casetti, L. Chiaraviglio, and A. Senacheribbe, "Planning UAV activities for efficient user coverage in disaster areas," Ad Hoc Networks, vol. 89, pp. 177-185, 2019.

[276] A. Merwaday, A. Tuncer, A. Kumbhar, and I. Guvenc, "Improved throughput coverage in natural disasters: Unmanned aerial base stations for public-safety communications," IEEE Vehicular Technology Magazine, vol. 11, no. 4, pp. 53-60, 2016.

[277] K. Guevara, M. Rodriguez, N. Gallo, G. Velasco, K. Vasudeva, and I. Guvenc, "UAV-based GSM network for public safety communications," in SoutheastCon 2015. IEEE, 2015, pp. 1-2.

[278] G. Tuna, B. Nefzi, and G. Conte, "Unmanned aerial vehicle-aided communications system for disaster recovery," Journal of Network and Computer Applications, vol. 41, pp. 27-36, 2014.

[279] Y. Du, K. Wang, K. Yang, and G. Zhang, "Energy-Efficient Resource Allocation in UAV Based MEC System for IoT Devices," in Proceedings of the IEEE Global Communications Conference (GLOBECOM). IEEE, 2018, pp. 1-6.

[280] M. F. Pinto, A. L. Marcato, A. G. Melo, L. M. Honório, and C. Urdiales, "A Framework for Analyzing Fog-Cloud Computing 
Cooperation Applied to Information Processing of UAVs," Wireless Communications and Mobile Computing, vol. 2019, 2019.

[281] S. Mahmoud and N. Mohamed, "Collaborative UAVs cloud," in Proceedings of the International Conference on Unmanned Aircraft Systems (ICUAS). IEEE, 2014, pp. 365-373.

[282] W. Chen, B. Liu, H. Huang, S. Guo, and Z. Zheng, "When UAV Swarm Meets Edge-Cloud Computing: The QoS Perspective," IEEE Network, vol. 33, no. 2, pp. 36-43, 2019.

[283] J. Zhang, L. Zhou, Q. Tang, E. C.-H. Ngai, X. Hu, H. Zhao, and J. Wei, "Stochastic Computation Offloading and Trajectory Scheduling for UAV-Assisted Mobile Edge Computing," IEEE Internet of Things Journal, 2018.

[284] F. Zhou, Y. Wu, H. Sun, and Z. Chu, "UAV-enabled mobile edge computing: Offloading optimization and trajectory design," in Proceedings of the IEEE International Conference on Communications (ICC). IEEE, 2018, pp. 1-6.

[285] N. Kalatzis, M. Avgeris, D. Dechouniotis, K. PapadakisVlachopapadopoulos, I. Roussaki, and S. Papavassiliou, "Edge Computing in IoT Ecosystems for UAV-Enabled Early Fire Detection," in Proceedings of the IEEE International Conference on Smart Computing (SMARTCOMP). IEEE, 2018, pp. 106-114.

[286] O. Bekkouche, T. Taleb, and M. Bagaa, "UAVs traffic control based on multi-access edge computing," in Proceedings of the IEEE Global Communications Conference (GLOBECOM). IEEE, 2018, pp. 1-6.

[287] X. Cao, J. Xu, and R. Zhangt, "Mobile Edge Computing for CellularConnected UAV: Computation Offloading and Trajectory Optimization," in Proceedings of the IEEE 19th International Workshop on Signal Processing Advances in Wireless Communications (SPAWC). IEEE, 2018, pp. 1-5.

[288] F. Zhou, Y. Wu, R. Q. Hu, and Y. Qian, "Computation rate maximization in UAV-enabled wireless-powered mobile-edge computing systems," IEEE Journal on Selected Areas in Communications, vol. 36, no. 9, pp. 1927-1941, 2018.

[289] Q. Fan and N. Ansari, "Towards traffic load balancing in drone-assisted communications for IoT," IEEE Internet of Things Journal, vol. 6 , no. 2, pp. 3633-3640, 2018.

[290] T. Yu, X. Wang, J. Jin, and K. McIsaac, "Cloud-orchestrated physical topology discovery of large-scale IoT systems using UAVs," IEEE Transactions on Industrial Informatics, vol. 14, no. 5, pp. 2261-2270, 2018.

[291] M. Mukherjee, R. Matam, L. Shu, L. Maglaras, M. A. Ferrag, N. Choudhury, and V. Kumar, "Security and privacy in fog computing: Challenges," IEEE Access, vol. 5, pp. 19293-19304, 2017.

[292] D. He, Y. Qiao, S. Chan, and N. Guizani, "Flight security and safety of drones in airborne fog computing systems," IEEE Communications Magazine, vol. 56, no. 5, pp. 66-71, 2018.

[293] N. Mohamed, J. Al-Jaroodi, I. Jawhar, H. Noura, and S. Mahmoud, "UAVFog: A UAV-based fog computing for Internet of Things," in Proceedings of the IEEE SmartWorld, Ubiquitous Intelligence \& Computing, Advanced \& Trusted Computed, Scalable Computing \& Communications, Cloud \& Big Data Computing, Internet of People and Smart City Innovation (SmartWorld/SCALCOM/UIC/ATC/CBDCom/IOP/SCI). IEEE, 2017, pp. 18.

[294] N. Chen, Y. Chen, Y. You, H. Ling, P. Liang, and R. Zimmermann, "Dynamic urban surveillance video stream processing using fog computing," in Proceedings of the IEEE second international conference on multimedia big data (BigMM). IEEE, 2016, pp. 105-112.

[295] N. Chen, Y. Chen, S. Song, C.-T. Huang, and X. Ye, "Smart urban surveillance using Fog Computing," in Proceedings of the IEEE/ACM Symposium on Edge Computing (SEC). IEEE, 2016, pp. 95-96.

[296] S. Jeong, O. Simeone, and J. Kang, "Mobile edge computing via a UAV-mounted cloudlet: Optimization of bit allocation and path planning," IEEE Transactions on Vehicular Technology, vol. 67, no. 3, pp. 2049-2063, 2018.

[297] S. Jeong, O. Simeone, and J. Kang, "Mobile cloud computing with a UAV-mounted cloudlet: optimal bit allocation for communication and computation," Iet Communications, vol. 11, no. 7, pp. 969-974, 2017.

[298] S. Y. M. Mahmoud and N. Mohamed, "Toward a cloud platform for UAV resources and services," in Proceedings of the IEEE Fourth Symposium on Network Cloud Computing and Applications (NCCA). IEEE, 2015, pp. 23-30.

[299] C. Luo, J. Nightingale, E. Asemota, and C. Grecos, "A UAV-cloud system for disaster sensing applications," in Proceedings of the IEEE 81st Vehicular Technology Conference (VTC Spring). IEEE, 2015, pp. $1-5$.
[300] N. Sharma, M. Magarini, D. N. K. Jayakody, V. Sharma, and J. Li, "Ondemand ultra-dense cloud drone networks: Opportunities, challenges and benefits," IEEE Communications Magazine, vol. 56, no. 8, pp. 85-91, 2018

[301] X. Wang, D. Li, C. Guo, X. Zhang, S. S. Kanhere, K. Li, and E. Tovar, "Eavesdropping and jamming selection policy for suspicious UAVs based on low power consumption over fading channels," Sensors, vol. 19 , no. 5, p. 1126, 2019.

[302] A. Y. Javaid, W. Sun, V. K. Devabhaktuni, and M. Alam, "Cyber security threat analysis and modeling of an Unmanned Aerial Vehicle system," in Proceedings of the IEEE Conference on Technologies for Homeland Security (HST). IEEE, 2012, pp. 585-590.

[303] L. Xiao, X. Lu, D. Xu, Y. Tang, L. Wang, and W. Zhuang, "UAV relay in VANETs against smart jamming with reinforcement learning," IEEE Transactions on Vehicular Technology, vol. 67, no. 5, pp. 4087-4097, 2018.

[304] N. I. Mowla, N. H. Tran, I. Doh, and K. Chae, "Federated LearningBased Cognitive Detection of Jamming Attack in Flying Ad-Hoc Network," IEEE Access, vol. 8, pp. 4338-4350, 2019.

[305] M. R. Manesh and N. Kaabouch, "Cyber Attacks on Unmanned Aerial System Networks: Detection, Countermeasure, and Future Research Directions," Computers \& Security, vol. 85, pp. 386-401, 2019.

[306] M. Elfar, H. Zhu, M. Cummings, and M. Pajic, "Security-aware synthesis of human-UAV protocols," in Proceedings of the International Conference on Robotics and Automation (ICRA). IEEE, 2019, pp. 8011-8017.

[307] H. Sedjelmaci, S. M. Senouci, and N. Ansari, "A hierarchical detection and response system to enhance security against lethal cyber-attacks in UAV networks," IEEE Transactions on Systems, Man, and Cybernetics: Systems, vol. 48, no. 9, pp. 1594-1606, 2017.

[308] Y. Guo, M. Wu, K. Tang, J. Tie, and X. Li, "Covert Spoofing Algorithm of UAV Based on GPS/INS-Integrated Navigation," IEEE Transactions on Vehicular Technology, vol. 68, no. 7, pp. 6557-6564, 2019.

[309] I. García-Magariño, R. Lacuesta, M. Rajarajan, and J. Lloret, "Security in networks of unmanned aerial vehicles for surveillance with an agentbased approach inspired by the principles of blockchain," Ad Hoc Networks, vol. 86, pp. 72-82, 2019.

[310] D. He, S. Chan, and M. Guizani, "Drone-assisted public safety networks: The security aspect," IEEE Communications Magazine, vol. 55, no. 8, pp. 218-223, 2017

[311] C. Rani, H. Modares, R. Sriram, D. Mikulski, and F. L. Lewis, "Security of Unmanned Aerial Vehicle systems against cyber-physical attacks," The Journal of Defense Modeling and Simulation, vol. 13, no. 3, pp. 331-342, 2016.

[312] R. Altawy and A. M. Youssef, "Security, privacy, and safety aspects of civilian drones: A survey," ACM Transactions on Cyber-Physical Systems, vol. 1, no. 2, p. 7, 2017.

[313] A. Kapitonov, S. Lonshakov, A. Krupenkin, and I. Berman, "Blockchain-based protocol of autonomous business activity for multiagent systems consisting of UAVs," in Proceedings of the Workshop on Research, Education and Development of Unmanned Aerial Systems (RED-UAS). IEEE, 2017, pp. 84-89.

[314] E. Asmare, N. Dulay, E. Lupu, M. Sloman, S. Calo, and J. Lobo, "Secure dynamic community establishment in coalitions," in Proceedings of the IEEE Military Communications Conference (MILCOM 2007). IEEE, 2007, pp. 1-7.

[315] A. Sanjab, W. Saad, and T. Başar, "Prospect theory for enhanced cyberphysical security of drone delivery systems: A network interdiction game," in Proceedings of the IEEE International Conference on Communications (ICC). IEEE, 2017, pp. 1-6.

[316] H. Sedjelmaci, S. M. Senouci, and N. Ansari, "A hierarchical detection and response system to enhance security against lethal cyber-attacks in UAV networks," IEEE Transactions on Systems, Man, and Cybernetics: Systems, vol. 48, no. 9, pp. 1594-1606, 2018.

[317] H. Lee, S. Eom, J. Park, and I. Lee, "UAV-aided secure communications with cooperative jamming," IEEE Transactions on Vehicular Technology, vol. 67, no. 10, pp. 9385-9392, 2018.

[318] F. Shi, J. Xia, Z. Na, X. Liu, Y. Ding, and Z. Wang, "Secure probabilistic caching in random multi-user multi-UAV relay networks," Physical Communication, vol. 32, pp. 31-40, 2019.

[319] F. Fei, Z. Tu, R. Yu, T. Kim, X. Zhang, D. Xu, and X. Deng, "Cross-layer retrofitting of UAVs against cyber-physical attacks," in Proceedings of the IEEE International Conference on Robotics and Automation (ICRA). IEEE, 2018, pp. 550-557.

[320] C. Li, Y. Xu, J. Xia, and J. Zhao, "Protecting secure communication under UAV smart attack with imperfect channel estimation," IEEE Access, vol. 6, pp. 76395-76401, 2018. 
[321] A. J. Kerns, D. P. Shepard, J. A. Bhatti, and T. E. Humphreys, "Unmanned aircraft capture and control via GPS spoofing," Journal of Field Robotics, vol. 31, no. 4, pp. 617-636, 2014.

[322] M. Yampolskiy, P. Horvath, X. D. Koutsoukos, Y. Xue, and J. Sztipanovits, "Taxonomy for description of cross-domain attacks on CPS," in Proceedings of the 2nd ACM international conference on High confidence networked systems. ACM, 2013, pp. 135-142.

[323] Q. Wang, Z. Chen, W. Mei, and J. Fang, "Improving physical layer security using UAV-enabled mobile relaying," IEEE Wireless Communications Letters, vol. 6, no. 3, pp. 310-313, 2017

[324] K. Yoon, D. Park, Y. Yim, K. Kim, S. K. Yang, and M. Robinson, "Security authentication system using encrypted channel on UAV network," in Proceedings of the first IEEE International Conference on Robotic Computing (IRC). IEEE, 2017, pp. 393-398.

[325] D. Rudinskas, Z. Goraj, and J. Stankūnas, "Security analysis of UAV radio communication system," Aviation, vol. 13, no. 4, pp. 116-121, 2009.

[326] H. Reyes, N. Gellerman, and N. Kaabouch, "A cognitive radio system for improving the reliability and security of UAS/UAV networks," in Proceedings of the IEEE Aerospace Conference. IEEE, 2015, pp. 1-9.

[327] D. P. Shepard, J. A. Bhatti, T. E. Humphreys, and A. A. Fansler, "Evaluation of smart grid and civilian UAV vulnerability to GPS spoofing attacks," in Proceedings of the Radionavigation Laboratory Conference, 2012.

[328] B. Li, Z. Fei, Y. Zhang, and M. Guizani, "Secure UAV communication networks over 5G," IEEE Wireless Communications, vol. 26, no. 5, pp. 114-120, 2019

[329] Y. Zhou, P. L. Yeoh, H. Chen, Y. Li, R. Schober, L. Zhuo, and B. Vucetic, "Improving physical layer security via a UAV friendly jammer for unknown eavesdropper location," IEEE Transactions on Vehicular Technology, vol. 67, no. 11, pp. 11 280-11 284, 2018.

[330] X. Sun, W. Yang, Y. Cai, R. Ma, and L. Tao, "Physical Layer Security in Millimeter Wave SWIPT UAV-Based Relay Networks," IEEE Access, 2019.

[331] E. S. Dawam, X. Feng, and D. Li, "Autonomous Arial Vehicles in Smart Cities: Potential Cyber-Physical Threats," in Proceedings of the IEEE 20th International Conference on High Performance Computing and Communications; IEEE 16th International Conference on Smart City; IEEE 4th International Conference on Data Science and Systems (HPCC/SmartCity/DSS). IEEE, 2018, pp. 1497-1505.

[332] H. Qin, Z. Meng, W. Meng, X. Chen, H. Sun, F. Lin, and M. H. Ang, "Autonomous Exploration and Mapping System Using Heterogeneous UAVs and UGVs in GPS-Denied Environments," IEEE Transactions on Vehicular Technology, vol. 68, no. 2, pp. 1339-1350, 2019.

[333] M. Garratt, L. T. Bui, H. Abbass et al., "Apprenticeship Bootstrapping: Inverse Reinforcement Learning in a Multi-Skill UAV-UGV Coordination Task," in Proceedings of the 17th International Conference on Autonomous Agents and MultiAgent Systems. International Foundation for Autonomous Agents and Multiagent Systems, 2018, pp. 2204-2206.

[334] H. X. Pham, H. M. La, D. Feil-Seifer, and M. C. Deans, "A distributed control framework of multiple unmanned aerial vehicles for dynamic wildfire tracking," IEEE Transactions on Systems, Man, and Cybernetics: Systems, no. 99, pp. 1-12, 2018.

[335] M. Khabbaz, J. Antoun, and C. Assi, "Modeling and Performance Analysis of UAV-Assisted Vehicular Networks," IEEE Transactions on Vehicular Technology, vol. 68, no. 9, pp. 8384-8396, 2019.

[336] M. A. Uddin, A. Mansour, D. L. Jeune, M. Ayaz, e.-H. M. Aggoune et al., "UAV-assisted dynamic clustering of wireless sensor networks for crop health monitoring," Sensors, vol. 18, no. 2, p. 555, 2018.

[337] M. Hu, W. Liu, J. Lu, R. Fu, K. Peng, X. Ma, and J. Liu, "On the joint design of routing and scheduling for vehicle-assisted multi-UAV inspection," Future Generation Computer Systems, vol. 94, pp. 214 223,2019

[338] O. S. Oubbati, M. Atiquzzaman, P. Lorenz, H. Tareque, and S. Hossain, "Routing in Flying Ad hoc Networks: Survey, Constraints and Future Challenge Perspectives," IEEE Access, vol. 7, pp. 81 057-81 105, 2019.

[339] M. B. Ghorbel, D. Rodríguez-Duarte, H. Ghazzai, M. J. Hossain, and H. Menouar, "Joint Position and Travel Path Optimization for Energy Efficient Wireless Data Gathering Using Unmanned Aerial Vehicles," IEEE Transactions on Vehicular Technology, vol. 68, no. 3, pp. 2165$2175,2019$.

[340] C. Caillouet, F. Giroire, and T. Razafindralambo, "Efficient data collection and tracking with flying drones," Ad Hoc Networks, vol. 89, pp. 35-46, 2019.

[341] H. Binol, E. Bulut, K. Akkaya, and I. Guvenc, "Time Optimal MultiUAV Path Planning for Gathering ITS Data from Roadside Units," in
Proceedings of the IEEE 88th Vehicular Technology Conference (VTCFall). IEEE, 2018, pp. 1-5.

[342] J. Liu, X. Wang, B. Bai, and H. Dai, "Age-optimal trajectory planning for UAV-assisted data collection," in Proceedings of the IEEE Conference on Computer Communications Workshops (INFOCOM WKSHPS). IEEE, 2018, pp. 553-558

[343] S. Sarkar, M. W. Totaro, and K. Elgazzar, "Intelligent drone-based surveillance: application to parking lot monitoring and detection," in Proceedings of the Unmanned Systems Technology XXI, vol. 11021. International Society for Optics and Photonics, 2019, p. 1102104.

[344] M. Hu, W. Liu, K. Peng, X. Ma, W. Cheng, J. Liu, and B. Li, "Joint routing and scheduling for vehicle-assisted multi-drone surveillance," IEEE Internet of Things Journal, 2018.

[345] G. Stamatescu, D. Popescu, and R. Dobrescu, "Cognitive radio as solution for ground-aerial surveillance through WSN and UAV infrastructure," in Proceedings of the 2014 6th International Conference on Electronics, Computers and Artificial Intelligence (ECAI). IEEE, 2014, pp. 51-56.

[346] S. Berrahal, J.-H. Kim, S. Rekhis, N. Boudriga, D. Wilkins, and J. Acevedo, "Border surveillance monitoring using quadcopter UAVaided wireless sensor networks," 2016

[347] G. Geraci, A. Garcia-Rodriguez, L. G. Giordano, D. López-Pérez, and E. Björnson, "Understanding UAV cellular communications: from existing networks to massive MIMO," IEEE Access, vol. 6, pp. 67853 $67865,2018$.

[348] S. Zhang, Y. Zeng, and R. Zhang, "Cellular-enabled UAV communication: Trajectory optimization under connectivity constraint," in Proceedings of the IEEE International Conference on Communications (ICC). IEEE, 2018, pp. 1-6.

[349] Z. Hu, Z. Zheng, L. Song, T. Wang, and X. Li, "UAV Offloading: Spectrum Trading Contract Design for UAV-Assisted Cellular Networks," IEEE Transactions on Wireless Communications, vol. 17, no. 9, pp. 6093-6107, 2018.

[350] D. López-Pérez, M. Ding, H. Li, L. G. Giordano, G. Geraci, A. GarciaRodriguez, Z. Lin, and M. Hassan, "On the Downlink Performance of UAV Communications in Dense Cellular Networks," in Proceedings of the IEEE Global Communications Conference (GLOBECOM). IEEE, 2018, pp. 1-7.

[351] Z. Xue, J. Wang, G. Ding, H. Zhou, and Q. Wu, "Maximization of data dissemination in UAV-supported internet of things," IEEE Wireless Communications Letters, vol. 8, no. 1, pp. 185-188, 2018.

[352] A. Ahmed, M. Awais, T. Akram, S. Kulac, M. Alhussein, and K. Aurangzeb, "Joint Placement and Device Association of UAV Base Stations in IoT Networks," Sensors, vol. 19, no. 9, p. 2157, 2019.

[353] R. Duan, J. Wang, C. Jiang, H. Yao, Y. Ren, and Y. Qian, "Resource Allocation for Multi-UAV Aided IoT NOMA Uplink Transmission Systems," IEEE Internet of Things Journal, 2019.

[354] N. Cheng, F. Lyu, W. Quan, C. Zhou, H. He, W. Shi, and X. Shen, "Space/Aerial-Assisted Computing Offloading for IoT Applications: A Learning-Based Approach," IEEE Journal on Selected Areas in Communications, vol. 37, no. 5, pp. 1117-1129, 2019.

[355] N. Athanasis, M. Themistocleous, K. Kalabokidis, and C. Chatzitheodorou, "Big Data Analysis in UAV Surveillance for Wildfire Prevention and Management," in Proceedings of the European, Mediterranean, and Middle Eastern Conference on Information Systems. Springer, 2018, pp. 47-58.

[356] K. M. Hasan, S. S. Newaz, and M. S. Ahsan, "Design and development of an aircraft type portable drone for surveillance and disaster management," International Journal of Intelligent Unmanned Systems, vol. 6, no. 3, pp. 147-159, 2018.

[357] C.-C. Chuang, J.-Y. Rau, M.-K. Lai, and C.-L. Shih, "Combining Unmanned Aerial Vehicles, and Internet Protocol Cameras to Reconstruct 3-D Disaster Scenes During Rescue Operations," Prehospital Emergency Care, pp. 1-6, 2018.

[358] A. Gao, Y. Hu, L. Li, and X. Li, "BP Network Control for Resource Allocation and QoS Ensurance in UAV Cloud," Journal of Sensors, vol. 2018, 2018.

[359] Y. Dong, M. Z. Hassan, J. Cheng, M. J. Hossain, and V. C. Leung, "An edge computing empowered radio access network with UAV-mounted FSO fronthaul and backhaul: Key challenges and approaches," IEEE Wireless Communications, vol. 25, no. 3, pp. 154-160, 2018.

[360] A. Gao, Y. Hu, W. Liang, Y. Lin, L. Li, and X. Li, "A QoE-Oriented Scheduling Scheme for Energy-Efficient Computation Offloading in UAV Cloud System," IEEE Access, 2019.

[361] A. R. Sobhy, A. T. Khalil, M. M. Elfaham, and A. Hashad, "UAV Cloud Operating System," in Proceedings of the MATEC Web of Conferences, vol. 188. EDP Sciences, 2018, p. 05011. 
[362] J. Sun, W. Wang, Q. Da, L. Kou, G. Zhao, L. Zhang, and Q. Han, "An Intrusion Detection Based on Bayesian Game Theory for UAV Network," in Proceedings of the 11th EAI International Conference on Mobile Multimedia Communications. ICST (Institute for Computer Sciences, Social-Informatics, 2018, pp. 56-67.

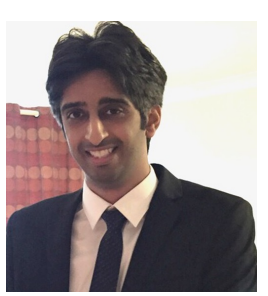

Bander A. Alzahrani is an assistance professor at King Abdulaziz University, Saudi Arabia. He completed his M.Sc. in Computer Security (2010), and his Ph.D. in Computer Science (2015), both from Essex University, United Kingdom. His research interests include Wireless sensor networks, Information centric networks, Bloom filter data structure and its applications, secure content routing, authentication protocols in IoT. Bander has published more than 27 research papers in International Journals and conferences.

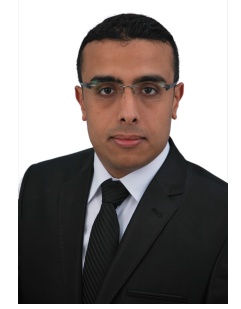

Omar Sami Oubbati is an Associate Professor at the Electronics departement, University of Laghouat, Algeria and a Research Assistant in the Computer Science and Mathematics Lab (LIM) at the same university. He received his degree of Engineer (2010), M.Sc. in Computer Engineering (2011) M.Sc. degree (2014), and a PhD in Computer Science (2018). From Oct. 2016 to Oct. 2017, he was a Visiting Student with the Laboratory of Computer Science, University of Avignon, France. His main research interests are in Flying and Vehicular ad hoc networks, Visible light communications, Energy efficiency and Internet of Things (IoT). He serves on the editorial board of Vehicular Communications Journal of Elsevier. He is a reviewer in many international journals and a TPC member in many international conferences. He is a member of the IEEE and IEEE Communications Society.

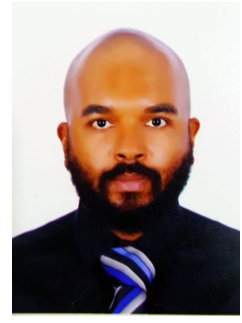

Ahmed Barnawi is currently a professor of information and communication technologies at Faculty of Computing and IT (FCIT) in King Abdulaziz University (KAU). $\mathrm{He}$ is the managing director of the KAU Cloud computing and Big Data Research group. He acquired his Phd from the University of Bradford, UK, in 2005 and his MSC from UMIST (University of Manchester), UK, in 2001. Prof. Barnawi acted as an associate and Visiting Professors in Canada and Germany. Prof. Barnawi is an active researcher with good research fund awards track. His research interest include Big data, cloud computing, future generation mobile systems, advanced mobile robotic applications and IT infrastructure architecture. He published near to 100 papers in peer reviewed journals.

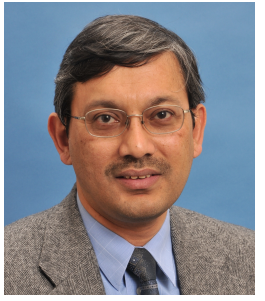

Mohammed Atiquzzaman received the M.S. and $\mathrm{Ph} . \mathrm{D}$. degrees in electrical engineering and electronics from the University of Manchester, U.K., in 1984 and 1987, respectively. He currently holds the Edith J. Kinney Gaylord Presidential Professorship with the School of Computer Science, University of Oklahoma, USA. His research has been funded by the National Science Foundation, National Aeronautics and Space Administration, U.S. Air Force, Cisco, and Honeywell. He co-authored Performance of TCP/IP Over ATM Networks and has authored over 300 refereed publications. His current research interests are in areas of transport protocols, wireless and mobile networks, ad hoc networks, satellite networks, power-aware networking, and optical communications. He CoChaired the IEEE High Performance Switching and Routing Symposium $(2003,2011)$, IEEE GLOBECOM and ICC $(2014,2012,2010,2009,2007$, and 2006), IEEE VTC (2013), and SPIE Quality of Service Over Next Generation Data Networks conferences (2001, 2002, and 2003). He was the Panels Co-Chair of INFOCOM'05, and has been on the program committee of many conferences, such as INFOCOM, GLOBECOM, ICCCN, ICCIT, Local Computer Networks, and serves on the review panels at the National Science Foundation. He was the Chair of the IEEE Communication Society Technical Committee on Communications Switching and Routing. He received the IEEE Communication Society's Fred W. Ellersick Prize and the NASA Group Achievement Award for outstanding work to further NASA Glenn Research Center's efforts in the area of the Advanced Communications/Air Traffic Management's Fiber Optic Signal Distribution for Aeronautical Communications project. He received from IEEE the 2018 Satellite and Space Communications Technical Recognition Award for valuable contributions to the Satellite and Space Communications scientific community. He also received the 2017 Distinguished Technical Achievement Award from IEEE Communications Society in recognition of outstanding technical contributions and services in the area of communications switching and routing. He is the Editor in-Chief of Journal of Networks and Computer Applications, the founding Editorin-Chief of Vehicular Communications, and serves served on the editorial boards of many journals, including IEEE Communications Magazine, Real Time Imaging Journal, International Journal of Communication Networks and Distributed Systems, Journal of Sensor Networks, and International Journal of Communication Systems.

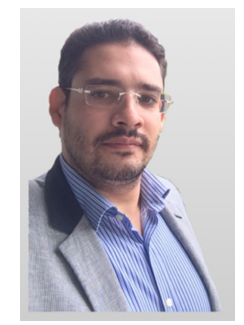

Daniyal Alghazzawi is a Professor in the Computing Information Systems Department and the Head of the Information Security Group at King Abdulaziz University. He received his BSc degree with honor in Computer Science from King Abdulaziz University in 1999. Then, he completed his MSc and PhD degrees in the field of Computer Science at the University of Kansas at the United States in 2007. He also received another MSc degree in Teaching and Leadership from University of Kansas in 2004 which helped him to develop his teaching and leadership skills. Since 2007, he served as the Head of Department for five years, and then he served as a Vice Dean of Development of the Deanship of Information Technology for two years. In 2010, he became an Honorary Lecturer at School of Computer Science and Electronic Engineering, at University of Essex in UK 